FTUV-99/20 IFIC-99/21

April 10, 1999

REVISED (shortened): July 13. Version to appear in NPB

hep-th/9904137

\title{
The geometry of branes and extended superspaces
}

\author{
C. Chryssomalakos ${ }^{1}$, J. A. de Azcárraga ${ }^{1}$, J. M. Izquierdo ${ }^{2}$ and

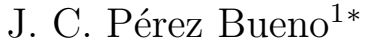 \\ 1 Departamento de Física Teórica, Universidad de Valencia \\ and IFIC, Centro Mixto Universidad de Valencia-CSIC \\ E-46100 Burjassot (Valencia), Spain \\ 2 Departamento de Física Teórica, Universidad de Valladolid \\ E-47011 Valladolid, Spain
}

\begin{abstract}
We argue that a description of supersymmetric extended objects from a unified geometric point of view requires an enlargement of superspace. To this aim we study in a systematic way how superspace groups and algebras arise from Grassmann spinors when these are assumed to be the only primary entities. In the process, we recover generalized spacetime superalgebras and extensions of supersymmetry found earlier. The enlargement of ordinary superspace with new parameters gives rise to extended superspace groups, on which manifestly supersymmetric actions may be constructed for various types of $p$-branes, including D-branes (given by Chevalley-Eilenberg cocycles) with their Born-Infeld fields. This results in a field/extended superspace democracy for superbranes: all brane fields appear as pull-backs from a suitable target superspace. Our approach also clarifies some facts concerning the origin of the central charges for the different $p$-branes.
\end{abstract}

* e-mail addresses: chryss@lie3.ific.uv.es, azcarrag@lie1.ific.uv.es, izquierd@fta.uva.es, pbueno@lie.ific.uv.es 


\section{Introduction}

As is well known, the existence of consistent classical actions for extended supersymmetric objects of spatial dimension $p$ is restricted to certain dimensions $D$ of spacetime. This is e.g. the case of the $p$-branes of the minimal or 'old' branescan [1], which restricts the actions to certain values $(D, p)$ for which there exists a Wess-Zumino (WZ) term. This is needed for the $\kappa$-symmetry of the full action that matches the physical bosonic and fermionic degrees of freedom on the worldvolume $W$, and the WZ term is given by a closed $(p+2)$-form which can be interpreted [2] as a Chevalley-Eilenberg [3] (CE) $(p+2)$-cocycle on superspace. The first classification of $p$-branes [1] was restricted to fields forming a scalar supermultiplet on $W$, consisting of scalars and spinors after gauging the $\kappa$-symmetry. The restriction to superspace coordinates $x^{\mu}, \theta^{\alpha}$ on $W$ was later removed with the addition of higher spin fields, vectors or antisymmetric tensors, forming vector or antisymmetric tensor supermultiplets on $W$. This, together with the Bose-Fermi matching conditions led to an enlargement of the possibilities (see [4, 5] and earlier references therein) for the classically allowed supermembranes. Recently, $p$ branes including an abelian vector gauge field on $W$ have been interpreted as (Dirichlet) $D$-branes [6] (see [7] for a review). Their kinetic term is described by a Born-Infeld type Lagrangian which replaces the usual Nambu-Goto one to accommodate the vector potential; in similarity with the $p$ branes in [1], there also exists a $\kappa$-symmetric worldvolume action [8] for them. The introduction of other objects such as $L$-branes (which have linear supermultiplets on $W$ ) [9] etc., have enlarged the number and types of $p$-branes. Finally, the emergence of a web of dualities among the five consistent 10-dimensional string theories, all presumably subsumed, together with $D=11$ supergravity, in the eleven dimensional $M$-theory (see, e.g. [10]) has led to the 'second superstring revolution' and to a change of the conventional views of supersymmetry. One version of the $M$-theory, $M$-atrix theory [11], even reinterprets spacetime coordinates as non-commuting matrices.

The existence of various extended objects for which there is no unified description suggests that, in the same way Minkowski space was enlarged to the superspace $\Sigma$ to treat bosons and fermions simultaneously, it may be necessary to extend $\Sigma$ further to accommodate in a unified point of view a number of the physical aspects mentioned above. In particular, one might hope to remove the need of defining fields directly on $W$ if an extended superspace $\tilde{\Sigma}$ is introduced, as it will be seen to be the case. This extension of $\Sigma$ is tantamount to enlarging the $D$-dimensional superPoincaré $s P$ to $\widetilde{s P}$ and to defining the extended superspace $\tilde{\Sigma}$ by the quotient $\widetilde{s P} / \operatorname{Spin}(1, D-1)$. Endowing $\tilde{\Sigma}$ with a supergroup structure means that there must exist new superalgebras going beyond the ordinary supersymmetry algebra, and several of them have been discussed in various contexts [12, 13, 14, 15, 16, 17, 18, 19, 20, 21, 22]. Our point of view, however, will be to assume that fermions (in the form of odd abelian spinor translations) are the only basic (i.e., initial) entities. We shall then look for the most general superspace groups that are allowed by group extension theory and discuss their consequences for a unified picture of superbranes. We find this path rather natural, but it is not the only one. Another possibility is to take the worldvolume supersymmetry of the $p$-branes into account by elevating the target superspace coordinates to worldvolume superfields [23, 24] (see also [9] and references therein), but we shall not follow this superembedding or 'double supersymmetry' approach.

Stated as above, the problem is first a mathematical one. Much in the same way that rigid superspace is itself a group extension, and hence supersymmetry is the result of the non-trivial cohomology of a certain odd superstranslation group $\operatorname{sTr}_{D}$ [25, 26], it is worth looking for all the possible group extensions of the various $\operatorname{sTr}_{D}$ (i.e., $\mathrm{s} \operatorname{Tr}_{D}=\left\{N=1, \mathrm{~s} \operatorname{Tr}_{11}, \mathrm{~s} \operatorname{Tr}_{10}\right.$, IIA, IIB, etc $\}$ ) to explore their rôle in more general theories. At the algebra level, the possible supersymmetry algebras were already investigated in [27] and, allowing tensor 'central' charges, in [28] (tensorial charges were also considered in [29, 30, 31]). But there is also a physical reason behind the mathematical extension problem. It is known that the quasi-invariance of a Lagrangian under a symmetry indicates that the (second) cohomology group is non-trivial, and that the symmetry group may hence be extended.円 This was exploited in 12] to extend the supersymmetry algebra for the supersymmetric extended objects by topological charges. For Lagrangians containing a quasi-invariant piece $\phi^{*}(b)$ constructed from a form $b$ on a group $\Sigma$ by pulling it back to a manifold $W$ by $\phi^{*}$, where $\phi: W \rightarrow \Sigma$, the extension may allow us, as it is the case with the WZ terms $b$ of supersymmetric objects, to obtain manifestly invariant

\footnotetext{
${ }^{1}$ For a detailed account of quasi-invariance, Noether currents, cohomology and extensions, see [26, 32].
} 
terms $\tilde{b}$ f by defining them on an extended group manifold $\tilde{\Sigma}$. In fact, it was shown in 16] that to every free differential algebra in [2] corresponds a new spacetime superalgebra, from which invariant forms can be found to define new WZ terms 3. We shall take the analysis of [16, 2, 19] further (see also [18]) by considering various superbrane types and by emphasizing the supergroup manifold point of view. Thus, we shall look for and introduce extended superspace groups $\tilde{\Sigma}$ in a systematic way (we restrict our attention here to rigid superspaces). The additional variables in these will determine symmetries to which (topological) charges may correspond via the standard Noether theorem. For the branes of the old branescan, these new variables will appear only in the WZ term and as a total differential. This will be different for the D-branes, for which we will obtain, nevertheless, that it is also possible to find an action defined on an extended superspace (thus removing the necessity of introducing directly worldvolume fields) with a WZ term given by a $\mathrm{CE}(p+2)$-cocycle. By showing that all these structures and extended superspaces $\tilde{\Sigma}$ follow from a basic odd translation group $\mathrm{sTr}_{D}$ defined by the Grassmann spinors of the specific theory, we may conclude that the $\tilde{\Sigma}$ 's (and the corresponding extended superPoincaré groups $\widetilde{s P}$ ) are in a way as fundamental as the standard one, and necessary for a proper description of the physics involved around $M$-theory and its six weak coupling limit corners. The new variables may be relevant in the search for superbrane actions, in the description of dualities or in the quantisation process.

This paper is organised as follows. Sec. 2 contains all central extensions of $s \operatorname{Tr}_{D}$, including ordinary superspace, for various dimensions, and its results are summarised in a table. Sec. 3 considers in general the inclusion of additional non-central generators. Sec. A is devoted to the structure of the new superspaces $\tilde{\Sigma}$ and provides a compact expression for the contribution to the Noether charges coming from the WZ terms of the various possible actions, once they are formulated on $\tilde{\Sigma}$. Sec. D shows how the simplest $D=10,11$ extended superspaces are relevant to construct a manifestly invariant WZ term, both for the Green-Schwarz superstring [33, 13] (which we will complete with an additional contribution), and for the supermembrane. We shall recover there the results of [16] and compute the topological charges which, in our approach, correspond to the new group variables. The question of the linearity of the group action is seen in Sec. 5.1 to be associated with a coboundary election. Sections 6 and 8 show how the case of the IIA D $p$-branes and $M 5$-brane may also be treated within the same framework i.e., how branes containing vector and tensor fields on $W$ may be defined directly on suitably extended superspaces. We shall argue, in fact, that the picture is general and that suitable target superspaces exist on which to define all the fields appearing in the $p$-brane actions, including the various vector (see also [22]), tensor, etc. worldvolume fields. This is tantamount to establishing a general fields/extended superspace democracy in which the worldvolume fields and the extended superspace variables are on the same footing, as it was already the case for the minimal branescan. Indeed this correspondence between coordinates and fields has occasionally been discussed in the past in other contexts (see [34, 35, 36, 37]). Sec. 17 contains a brief discussion of the origin of the contributions to the Noether charges in the D-branes case [38] in our approach. Finally, an Appendix complements the general theory of non-central extensions of superspace in Sec. 3 and gives the proof of some needed $\Gamma$-matrix identities.

\section{Central extensions and their superspaces}

\subsection{Standard superspace as a central extension}

Let $\theta$ be an arbitrary Grassmann spinor in a $D$-dimensional spacetime. Its components $\theta^{\alpha}\left(2^{[D / 2]}\right.$ where $[D / 2]$ denotes the integer part of $D / 2$, or $2^{(D / 2)-1}$ in the Weyl case) determine an abelian group of supertranslations, generically denoted $\mathrm{sTr}_{D}$, with group composition law

$$
\theta^{\prime \prime \alpha}=\theta^{\prime \alpha}+\theta^{\alpha}
$$

\footnotetext{
${ }^{2}$ This is not always possible. When the group $G$ is simple, the extension appears only at the loop algebra (of charge densities) level, as for the $s u(2)$ Kac-Moody algebra for a WZW model, and disappears for the algebra of charges, as required by Whitehead's lemma.

${ }^{3}$ Although it may be argued that these invariant terms should no longer be called WZ terms, we shall retain this name for them.
} 
When the Lorentz part is considered explicitly, there is an action $\rho$ of $\operatorname{Spin}(1, D-1)$ on $\operatorname{sTr}_{D}$ and the relevant group becomes $\operatorname{sTr}_{D} \circ \operatorname{Spin}(1, D-1)$, where $\circ$ indicates semidirect product. Then (2.1) is replaced by

$$
\theta^{\prime \prime}=\theta^{\prime}+\rho(A) \theta \quad, \quad A^{\prime \prime}=A^{\prime} A \quad,
$$

where $A \in \operatorname{Spin}(1, D-1)$ and $\rho(A)$ is the appropriate spin representation. The spinor $\theta$ is often restricted to be of some specific type, usually minimal (e.g., Majorana (M), Weyl (W) or MajoranaWeyl (MW), when possible); it may carry an additional index $i=1, \ldots, N$ if there is more than one supersymmetry. Associated with (2.1) is the abelian Lie superalgebra $\left\{D_{\alpha}, D_{\beta}\right\}=0$ t, which can also be described in terms of the left-invariant (LI) one-forms $\Pi^{\alpha}=d \theta^{\alpha}$ and the trivial Maurer-Cartan (MC) equation

$$
d \Pi^{\alpha}=0
$$

Extending $\operatorname{sTr}_{D}$ by the Minkowski translations $x^{\mu}, \mu=0, \ldots, D-1$ leads to standard (rigid) superspace [25, 26]. Let us adopt the free differential algebra (FDA) point of view to discuss the extension problem, since forms are especially convenient in the construction of actions for extended objects.

Let $\theta^{\alpha}$ be Majorana, and consider the two-form $\left(C \Gamma^{\mu}\right)_{\alpha \beta} \Pi^{\alpha} \wedge \Pi^{\beta}$ on $\operatorname{sTr}_{D}$. It defines a non trivial CE two-cocycle on the superalgebra of $\operatorname{sTr}_{D}$, i.e. it is left-invariant (LI), closed and not given by the differential of a LI one-form. Since by construction the two-cocycle transforms as a Lorentz vector, it is consistent to extend the FDA $(2.3)$ by a one-form $\Pi^{\mu}$ such that

$$
d \Pi^{\mu}=\frac{1}{2}\left(C \Gamma^{\mu}\right)_{\alpha \beta} \Pi^{\alpha} \Pi^{\beta}
$$

(we omit the wedge product henceforth). The above extension immediately implies $\left\{D_{\alpha}, D_{\beta}\right\}=$ $\left(C \Gamma^{\mu}\right)_{\alpha \beta} X_{\mu}$, with $X_{\mu}$ central. One still has to relate the newly introduced one-form to the coordinate $x^{\mu}$. We define

$$
\Pi^{\mu}=d x^{\mu}+\frac{1}{2}\left(C \Gamma^{\mu}\right)_{\alpha \beta} \theta^{\alpha} d \theta^{\beta}
$$

and choose the transformation law for $x^{\mu}$ so that $\Pi^{\mu}$ is LI

$$
x^{\prime \prime \mu}=x^{\prime \mu}+x^{\mu}-\frac{1}{2}\left(C \Gamma^{\mu}\right)_{\alpha \beta} \theta^{\prime \alpha} \theta^{\beta} .
$$

This gives rigid superspace $\Sigma$, parametrized by $\left(\theta^{\alpha}, x^{\mu}\right)$ and with group law given by (2.1) and (2.6).

The above simple example exhibits already the key features of the extension algorithm. Given a particular FDA to be extended, one identifies in general a non-trivial two-cocycle of a desired Lorentz covariant nature and introduces a new LI one-form, the differential of which is given by the cocycle. The new form (here, (2.5)) together with the MC equations (here, eqns. (2.3) and (2.4)) automatically define by duality an extended Lie algebra. The new LI one-form is given by the sum of the differential of the new group parameter and the potential one-form of the CE two-cocycle on $\mathrm{sTr}_{D}$, which is not LI. Finally, the transformation properties of the new coordinate are fixed so as to guarantee the left invariance of the new one-form, while those of the original manifold are unmodified. The additional one-form can be made LI only if it is defined on the extended superspace manifold $\tilde{\Sigma}$. The new (central) generator, associated with translations along the new coordinates, modifies the r.h.s. of the original commutators of the algebra. Since adding (2.4) to (2.3) involves a central extension, we could have introduced a dimensionful constant of $\Pi^{\mu}$ are fixed to be $\left[\Pi^{\alpha}\right]^{2}=\left[\theta^{\alpha}\right]^{2}$. We shall, as usual, take $\left[\theta^{\alpha}\right]=L^{\frac{1}{2}}$ so that $\left[\Pi^{\mu}\right]=L$.

\footnotetext{
${ }^{4}$ Since we shall be considering left-invariant (LI) generators and forms, we shall use here D's (rather than $Q$ 's) to denote the generators of the right translations (the $Q$ 's being realized as the right-invariant (RI) generators of the left translations). This distinction is of course irrelevant for an abelian group such as (2.1) but it is not so when non-abelian parts are added (nevertheless, the corresponding structure constants differ only in a sign). Furthermore, LI and RI generators commute, $\{Q, D\}=0$. We may look at the $D$ 's as covariant derivatives and at the $Q$ 's as the generators of the (left) supersymmetry transformations; see Sec. 1.

${ }^{5}$ The term refers to an algebra generated by differential forms which is closed under the action of $d$ [39]. For early physical applications of FDA in supersymmetry see [40, 41] and references therein.

6 The value of this constant determines the specific element in cohomology space that characterizes the central extension.
} 
If we add the Lorentz group, the result must reflect the action $\sigma$ of $\operatorname{Spin}(1, D-1)$ on the extension cocyle (see, e.g. [26], Sec. 5.3), but we shall not consider explicitly the effect of the simple part of the algebra which, apart from extracting the various tensor-valued second cohomology groups from that of the trivial $(\sigma=0)$ action $H_{0}^{2}\left(\operatorname{sTr}_{D}\right)$ (see below), plays no essential rôle in our discussion once only Lorentz covariant objects are used. Thus, central means, where appropriate, central up to Lorentz transformations.

The extension procedure described above can be applied more than once - there are two basic patterns one may follow in this case. One can start in each step with the same original manifold $\mathrm{sTr}_{D}$, and keep adding two-cocycles and central generators or, in each step, one can consider the result of the previous extension as the starting manifold. In the first case (Sec. 2.2) all new generators remain central and appear only at the r.h.s. of the original $\{D, D\}$ anticommutator. In the second case, a richer structure emerges since the generators introduced at each step can, in principle, modify all previous commutators. We shall give the details of this second construction in Sec. 3 .

\subsection{Maximal central extensions of superspace}

Let $\theta^{\alpha}$ be Majorana. We may obtain additional Lorentz tensors, leading to new central charges, by considering

$$
d \Pi^{\mu_{1} \ldots \mu_{p}} \equiv \frac{1}{2}\left(C \Gamma^{\mu_{1} \ldots \mu_{p}}\right)_{\alpha \beta} \Pi^{\alpha} \Pi^{\beta}, \quad\left(\Gamma^{\mu_{1} \ldots \mu_{p}}=\Gamma^{\left[\mu_{1}\right.} \Gamma^{\mu_{2}} \ldots \Gamma^{\left.\mu_{p}\right]} \equiv \frac{1}{p !} \epsilon_{\nu_{1} \ldots \nu_{p}}^{\mu_{1} \ldots \mu_{p}} \Gamma^{\nu_{1}} \ldots \Gamma^{\nu_{p}}\right),
$$

where] $C \Gamma^{\mu} C^{-1}=-\Gamma^{\mu T}$. The antisymmetry in the Lorentz indices is needed to rule out trivial dependences coming from the fact that $\left\{\Gamma^{\mu}, \Gamma^{\nu}\right\}=2 \eta^{\mu \nu}$. The left invariance of the new forms in (2.7) requires new group parameters $\varphi^{\mu_{1} \ldots \mu_{p}}$ so that (cf. (2.5))

$$
\Pi^{\mu_{1} \ldots \mu_{p}}=d \varphi^{\mu_{1} \ldots \mu_{p}}+\frac{1}{2}\left(C \Gamma^{\mu_{1} \ldots \mu_{p}}\right)_{\alpha \beta} \theta^{\alpha} \Pi^{\beta}
$$

The superalgebra generator (LI vector field) $Z_{\mu_{1} \ldots \mu_{p}}$, corresponding to $\Pi^{\mu_{1} \ldots \mu_{p}}$, is realized by $Z_{\mu_{1} \ldots \mu_{p}}=$ $\partial / \partial \varphi^{\mu_{1} \ldots \mu_{p}}$ on the extended group manifold.

At this stage there are no restrictions coming from the Jacobi identity, equivalent to $d\left(d \Pi^{\mu_{1} \ldots \mu_{p}}\right)=$ 0 , which follows trivially from $d \Pi^{\alpha}=0$. This is an alternative way of stating that the $p$-tensor-valued mapping on $\operatorname{sTr}_{D} \otimes \mathrm{sTr}_{D}$,

$$
\xi^{\mu_{1} \ldots \mu_{p}}\left(\theta^{\prime}, \theta\right)=\theta^{\prime \alpha}\left(C \Gamma^{\mu_{1} \ldots \mu_{p}}\right)_{\alpha \beta} \theta^{\beta} \quad,
$$

satisfies trivially the two-cocycle condition

$$
\xi\left(\theta, \theta^{\prime}\right)+\xi\left(\theta+\theta^{\prime}, \theta^{\prime \prime}\right)=\xi\left(\theta, \theta^{\prime}+\theta^{\prime \prime}\right)+\xi\left(\theta^{\prime}, \theta^{\prime \prime}\right) .
$$

The symmetry of $\left(C \Gamma^{\mu_{1} \ldots \mu_{p}}\right)_{\alpha \beta}$ is needed to prevent the two-cocycle (2.9) from being trivial (i.e., a two-coboundary), since $\eta(\theta)=\theta^{\alpha}\left(C \Gamma^{\mu_{1} \ldots \mu_{p}}\right)_{\alpha \beta} \theta^{\beta}$ on $\operatorname{sTr}_{D}$, which might generate $\xi$ through $\xi_{\text {cob }}\left(\theta^{\prime}, \theta\right) \equiv$ $\eta\left(\theta^{\prime}+\theta\right)-\eta\left(\theta^{\prime}\right)-\eta(\theta)$, is identically zero. Thus, (2.9) defines a non-trivial extension. For a given spacetime dimension $D$, the symmetry condition restricts the rank of the tensors that are allowed in (2.7). Hence, the problem of finding all central extensions of the algebra $\left\{D_{\alpha}, D_{\beta}\right\}=0$ (or of the Lie FDA (2.3)) is reduced to finding a basis of the symmetric space $\Pi^{(\alpha} \otimes \Pi^{\beta)}$ in terms of tensors $\left(C \Gamma^{\mu_{1} \ldots \mu_{p}}\right)_{\alpha \beta}$ symmetric in $\alpha, \beta$; they define the Lie algebra CE two-cocycles $\Pi^{\alpha}\left(C \Gamma^{\mu_{1} \ldots \mu_{p}}\right)_{\alpha \beta} \Pi^{\beta}$.

When $D$ is even, the space of matrices with indices $(\alpha \beta)$ is $2^{D}$-dimensional. Since $2^{D}=\sum_{p=0}^{D}\left(\begin{array}{l}D \\ p\end{array}\right)$, a basis for this space is provided by the $\left(2^{D}-1\right)$ matrices given by the Lorentz tensors $\Gamma^{\mu_{1} \ldots \mu_{p}}$ of rank $1 \leq p \leq D$ plus the unit matrix. For $D$ odd, the spinors have dimension $2^{(D-1) / 2}$ and, since $2^{D-1}=\sum_{p=0}^{(D-1) / 2}\left(\begin{array}{l}D \\ p\end{array}\right)$, a basis is provided by the $\left(2^{D-1}-1\right)$ matrices given by the tensors $\Gamma^{\mu_{1} \ldots \mu_{p}}$ of rank $1 \leq p \leq(D-1) / 2$ plus the unit matrix. The difference is a consequence of the fact that, for any $D$,

$$
\Gamma^{\mu_{1} \ldots \mu_{p}} \Gamma^{D+1} \propto \epsilon^{\mu_{1} \ldots \mu_{D}} \Gamma_{\mu_{p+1} \ldots \mu_{D}}
$$

\footnotetext{
${ }^{7}$ We adopt $C \equiv C_{-}$for simplicity. By not considering $C_{+} \Gamma^{\mu} C_{+}^{-1}=\Gamma^{\mu T}$ we rule out, e.g., the pseudoMajorana spinors that exist for $D=8,9[\bmod 8]($ see $[42])$.
} 
where $\Gamma^{D+1}$ is the chirality matrix. For $D$ odd, $\Gamma^{D+1} \propto 1$, and only the tensors of rank $0 \leq p \leq$ $(D-1) / 2$ are linearly independent.

For $D$ even, $C \Gamma^{\mu_{1} \ldots \mu_{p}}$ satisfies (see e.g., [43])

$$
\begin{gathered}
\left(C \Gamma^{\mu_{1} \ldots \mu_{p}}\right)=\epsilon(-1)^{(p-1)(p-2) / 2}\left(C \Gamma^{\mu_{1} \ldots \mu_{p}}\right)^{T} \quad, \quad \mu=0,1, \ldots, D-1 \\
\epsilon=-\sqrt{2} \cos \frac{\pi}{4}(D+1)
\end{gathered}
$$

Thus, $\epsilon=1(-1)$ for $D=2,4(6,8)[\bmod 8]$ so that $\left(C \Gamma^{\mu_{1} \ldots \mu_{p}}\right)_{\alpha \beta}$ is symmetric for $p=1,2[\bmod 4]$ if $D=2,4[\bmod 8]$ and for $p=3,4$ if $D=6,8[\bmod 8]$. For $D$ odd, it turns out that the same condition, $\epsilon(-1)^{(p-1)(p-2) / 2}=1$, holds for $D=3[\bmod 4]$ with $\epsilon=-\sqrt{2} \cos \frac{\pi}{4} D$. We have excluded here (somewhat arbitrarily) the $D=5,9$ cases because in these dimensions no $C$ such that $C \Gamma^{\mu} C^{-1}=-\Gamma^{\mu T}$ exists.

The number of cohomology spaces $H_{\sigma}^{2}\left(\operatorname{sir}_{D} \circ \operatorname{Spin}(1, D-1)\right)$ for various $\mathrm{s}_{D}$ groups is given in the table. As a two-form, the various CE two-cocycles are given by $d \theta\left(C \Gamma^{\mu_{1} \ldots \mu_{p}}\right) d \theta$. The corresponding new generators $Z_{\mu_{1} \ldots \mu_{p}}$ are all central, as is $X_{\mu}$ itself. They are on the same footing and may thought of as generalised momenta. Each of the resulting extensions defines an extended superspace group; we will denote them generically by $\tilde{\Sigma}$.

The table also includes the cases in which the spinor is Majorana-Weyl or complex (Dirac and Weyl). If the spinor is complex the independent tensors $\Gamma^{0} \Gamma^{\mu_{1} \ldots \mu_{p}}$ may appear. The effect of considering Weyl spinors is taken into account by introducing a chiral projector $\left(\mathcal{P}_{+}\right.$, say).

The different extended supersymmetry algebras can be easily found from the results in the table. We shall only give below two examples which contain formulae that will be explicitly used later on. To avoid cumbersome factorials, we use a normalization of the generators which is tantamount to defining the duality relations by $\Pi^{\mu_{1} \ldots \mu_{p}}\left(Z_{\nu_{1} \ldots \nu_{p}}\right)=\frac{1}{p !} \epsilon_{\nu_{1} \ldots \nu_{p}}^{\mu_{1} \ldots \mu_{p}}$ so that $\Pi^{\mu_{1} \ldots \mu_{p}}\left(C \Gamma^{\nu_{1} \ldots \nu_{p}} Z_{\nu_{1} \ldots \nu_{p}}\right)=C \Gamma^{\mu_{1} \ldots \mu_{p}}$.

\subsection{Applications}

\subsection{1 $\mathrm{N}=1$ theory extended superspace}

For $D$ even, the basic spinors in (2.1) may be reduced to $2^{D / 2-1}$-dimensional Weyl spinors, and the discussion of the possible $H_{\sigma}^{2}\left(\operatorname{sTr}_{D} \circ \operatorname{Spin}(1, D-1)\right)$ spaces must take this into account. Let $D=2[\bmod 8]$ and let $\theta^{\alpha}$ be MW. The symmetry of $\left(C \Gamma^{\mu_{1} \ldots \mu_{p}} \mathcal{P}_{ \pm}\right)_{\alpha \beta}$ is now achieved if both $C \Gamma^{\mu_{1} \ldots \mu_{p}}$ and $C \Gamma^{\mu_{1} \ldots \mu_{p}} \Gamma^{D+1}$ (or, on account of (2.11), $C \Gamma^{\mu_{1} \ldots \mu_{D-p}}$ ) are symmetric. Hence, there are central charges for $D=2, p=1$ and $D=10, p=1,5,9$ (i.e., $p=1[\bmod 4]$ ). As a result, the $D=10, N=1$ extended superspace algebra has the form

$$
\left\{D_{\alpha}^{+}, D_{\beta}^{+}\right\}=\left(C \Gamma^{\mu} \mathcal{P}_{+}\right)_{\alpha \beta} X_{\mu}+\left(C \Gamma^{\mu_{1} \ldots \mu_{5}} \mathcal{P}_{+}\right)_{\alpha \beta} Z_{\mu_{1} \ldots \mu_{5}}+\left(C \Gamma^{\mu_{1} \ldots \mu_{9}} \mathcal{P}_{+}\right)_{\alpha \beta} Z_{\mu_{1} \ldots \mu_{9}} \quad .
$$

Due to $\mathcal{P}_{+}$and to (2.11), the first and last term in the r.h.s may be grouped into a single one, $\left(C \Gamma^{\mu} \mathcal{P}_{+}\right)\left(X_{\mu}+Z_{\mu}\right)$; classically, $Z_{\mu}$ may be absorbed by redefining $X_{\mu}$ and the previous analysis shows that the vector-valued cohomology space is one-dimensional. The second term may be rewritten as $\left(C \Gamma^{\mu_{1} \ldots \mu_{5}}\right) Z_{\mu_{1} \ldots \mu_{5}}^{+}$where $Z_{\mu_{1} \ldots \mu_{5}}^{+}$is a self-dual 5-tensor [28], $Z_{\mu_{1} \ldots \mu_{5}}^{+}=\frac{1}{2}\left(Z_{\mu_{1} \ldots \mu_{5}}+\epsilon_{\mu_{\ldots} \ldots \mu_{5}}{ }^{\mu_{6} \ldots \mu_{10}} Z_{\mu_{6} \ldots \mu_{10}}\right)$, with half the number of components of $Z_{\mu_{1} \ldots \mu_{5}}$. As a result, the degrees of freedom in eqn. (2.13) match: $\left(\begin{array}{c}16 \\ 2\end{array}\right)+16=136=10+\frac{1}{2}\left(\begin{array}{c}10 \\ 5\end{array}\right)$ (see table). But in general $Z_{\mu}$ cannot be reabsorbed, since the Green-Schwarz action for the heterotic superstring produces such a contribution to the algebra [12], of an origin different from that of $X_{\mu}$. Mathematically, this corresponds to the fact that the group parameters are different for $X_{\mu}$ (translations $x^{\mu}$ ) and $Z_{\mu}\left(\varphi^{\mu}\right)$; they are locally equivalent, much in the same way $\mathbb{R} \sim S^{1}$ locally, but they are different globally. We may, however, achieve the symmetry under the exchange of $X_{9}$ and $Z_{9}$ (say) when the 9-direction is a circle of radius $R$. Then the spectra of $X_{9}$ and $Z_{9}$ are isomorphic under the T-duality exchange $R \rightarrow 1 / R$ (see [45, 46]).

The FDA form of the $D=10, N=1$ superalgebra (2.13) is given by the MC relations

$$
d \Pi^{\alpha}=0, \quad d \Pi^{\mu}=\frac{1}{2}\left(C \Gamma^{\mu}\right)_{\alpha \beta} \Pi^{\alpha} \Pi^{\beta}, \quad d \Pi^{\mu_{1} \ldots \mu_{5}}=\frac{1}{2}\left(C \Gamma^{\mu_{1} \ldots \mu_{5}}\right)_{\alpha \beta} \Pi^{\alpha} \Pi^{\beta},
$$

where $\Pi^{\alpha}, \Pi^{\mu}$ and $\Pi^{\mu_{1} \ldots \mu_{5}}$ are defined as $(\alpha=1, \ldots, 32)$

$$
\Pi^{\alpha}=\mathcal{P}_{+} d \theta^{\alpha}, \quad \Pi^{\mu}=d x^{\mu}+\frac{1}{2}\left(C \Gamma^{\mu}\right)_{\alpha \beta} \theta^{\alpha} \Pi^{\beta}, \quad \Pi^{\mu_{1} \ldots \mu_{5}}=d \varphi^{\mu_{1} \ldots \mu_{5}}+\frac{1}{2}\left(C \Gamma^{\mu_{1} \ldots \mu_{5}}\right)_{\alpha \beta} \theta^{\alpha} \Pi^{\beta} .
$$




\begin{tabular}{|c|c|c|c|c|c|c|c|c|c|c|}
\hline 1 & 2 & 3 & & 4 & & 5 & 6 & 7 & 8 & 9 \\
\hline$D$ & $n=2^{[D / 2]}$ & $\frac{n(n+1)}{2}$ & $\begin{array}{l}\text { rea } \\
\text { ple } \\
\text { spil }\end{array}$ & $\begin{array}{l}n^{\prime} \\
\text { dil } \\
\text { or }\end{array}$ & $\begin{array}{l}{[\mathrm{com}-} \\
\text { of }\end{array}$ & $\begin{array}{l}\text { rank } p \text { of symmet- } \\
\text { ric } \quad\left(C \Gamma^{\mu_{1} \ldots \mu_{p}}\right)_{\alpha \beta} \\
\left\{\left(C \Gamma^{\mu_{1} \ldots \mu_{p}} \mathcal{P}_{+}\right)_{\alpha \beta}\right\}\end{array}$ & $\begin{array}{l}\operatorname{dim}\left(C \Gamma^{\mu_{1} \ldots \mu_{p}}\right) \\
\left\{\operatorname{dim}\left(C \Gamma^{\mu_{1} \ldots \mu_{p}} \mathcal{P}_{+}\right)\right\} ; \text {total } \\
\text { real }[\text { complex] dimension }\end{array}$ & $\begin{array}{l}\text { rank } \\
\left(\Gamma^{0} \Gamma^{\mu_{1} \ldots \mu_{p}}\right) \\
\left\{\left(\Gamma^{0} \Gamma^{\mu_{1} \ldots \mu_{p}} \mathcal{P}_{+}\right)\right\}\end{array}$ & $\begin{array}{l}\operatorname{dim}\left(\Gamma^{0} \Gamma^{\mu_{1} \ldots \mu_{p}}\right) \\
\left\{\operatorname{dim}\left(\Gamma^{0} \Gamma^{\mu_{1} \ldots \mu_{p}} \mathcal{P}_{+}\right)\right\}\end{array}$ & $\begin{array}{l}\text { real } H_{\sigma}^{2}\left(\mathrm{sTr}_{D} \quad \circ\right. \\
\operatorname{Spin}(1, D-1)) \text { spaces } \\
\left(\operatorname{dim} H_{0}^{2}\left(\mathrm{~s}_{D}\right)\right)\end{array}$ \\
\hline 2 & 2 & 3 & 1 & & MW & $\{1\}$ & $\left\{\frac{1}{2} 2 ; 1\right\}$ & \multirow{4}{*}{\multicolumn{2}{|c|}{$\begin{array}{c}\text { do not contribute further } \\
\text { in the real case }\end{array}$}} & $1 \quad(1)$ \\
\hline 2 & 2 & 3 & 2 & & M & 1,2 & 2,$1 ; 3$ & & & $(3)$ \\
\hline 3 & 2 & 3 & 2 & & $\mathbf{M}$ & 1 & $3 ; 3$ & & & $(3)$ \\
\hline 4 & 4 & 10 & 4 & & $\mathbf{M}$ & 1,2 & 4,$6 ; 10$ & & & $(10)$ \\
\hline 6 & 8 & 36 & 8 & {$[4]$} & $\mathbf{W}$ & $\{3\}$ & $\left\{\frac{1}{2} 20 ; 20[10]\right\}$ & $\{1,3,5\}$ & $\left\{6, \frac{1}{2} 20 ; 16\right\}$ & $(36)$ \\
\hline 6 & 8 & 36 & 16 & [8] & $\mathrm{D}$ & $0,3,4$ & $1,20,15 ; 72[36]$ & $0,1,2,3,4,5,6$ & $1,6,15,20,15,6,1 ; 64$ & $(136)$ \\
\hline 7 & 8 & 36 & 16 & [8] & $\mathbf{D}$ & 0,3 & 1,$35 ; 72[36]$ & $0,1,2,3$ & $1,7,21,35 ; 64$ & $(136)$ \\
\hline 8 & 16 & 136 & 16 & [8] & $\mathbf{W}$ & $\{0,4,8\}$ & $\left\{1, \frac{1}{2} 70 ; 72[36]\right\}$ & $\{1,3,5,7\}$ & $\{8,56 ; 64\}$ & $(136)$ \\
\hline 8 & 16 & 136 & 32 & {$[16]$} & $\mathrm{D}$ & $0,3,4,7,8$ & $1,56,70,8,1 ; 272[136]$ & $0,1,2,3,4,5,6,7,8$ & $1,8,28,56,70,56,28,8,1 ; 256$ & $(528)$ \\
\hline 10 & 16 & 136 & 16 & & MW & $\{1,5,9\}$ & $\left\{10, \frac{1}{2} 252 ; 136\right\}$ & \multirow{4}{*}{\multicolumn{2}{|c|}{$\begin{array}{c}\text { do not contribute further } \\
\text { in the real case }\end{array}$}} & $(136)$ \\
\hline 10 & 32 & 528 & 32 & & $\mathrm{M}$ & $1,2,5,6,9,10$ & $10,45,252,210,10,1 ; 528$ & & & $(528)$ \\
\hline 11 & 32 & 528 & 32 & & M & $1,2,5$ & $11,55,462 ; 528$ & & & $(528)$ \\
\hline 12 & 64 & 2080 & 64 & & $\mathbf{M}$ & $1,2,5,6,9,10$ & $12,66,792,924,220,66 ; 2080$ & & & $(2080)$ \\
\hline
\end{tabular}

Some Lie algebra second cohomology groups for $s \operatorname{Tr}_{D}$ (minimal spinors are in boldface). $n$ is the complex dimension of a Dirac spinor, equal to the real dimension of Majorana spinors for $D=2,3,4[\bmod 8]$. The fourth column gives the dimension of the spinor indicated. The fifth and sixth column give the ranks for which $\left(C \Gamma^{\mu_{1} \ldots \mu_{p}}\right)_{\alpha \beta}$ (or $\left(C \Gamma^{\mu_{1} \ldots \mu_{p}} \mathcal{P}_{+}\right)_{\alpha \beta}$ ) are symmetric (as deduced from $(2.12)$ ) and the dimension of these Lorentz tensors; $C$ itself is symmetric in $D=6,7,8$ [mod 8 ]. The seventh and eigth columns do the same for the additional tensors $\left(\Gamma^{0} \Gamma^{\mu_{1} \ldots \mu_{p}}\right)\left(\Gamma^{0} \Gamma^{\mu_{1} \ldots \mu_{p}} \mathcal{P}_{+}\right)$appearing in the complex spinor case. These hermitian (adding $i$ when needed) tensors are limited by duality (eqn. (2.11)) in the odd $(D=7)$ case and to odd rank by the presence of $\mathcal{P}_{+}$in the Weyl case. The $\frac{1}{2}$ indicates halving due to self-duality. The number of real cohomology groups $H_{\sigma}^{2}\left(\operatorname{sTr}_{D} \circ \operatorname{Spin}(1, D-1)\right)$ is given by the first number in the last column. These spaces are the relevant $(i . e . t e n s o r i a l)$ ones, once the Lorentz symmetry is considered since in this case $\operatorname{sTr}_{D} \circ \operatorname{Spin}(1, D-1)$ (rather than $\operatorname{sTr}_{D}$ ) is the group to be extended. The action $\sigma$ of $\operatorname{Spin}(1, D-1)$ on the extension cocycles is automatically taken into account by using only Lorentz covariant objects for them. The bracketed number in the last column ignores the Lorentz part and, as a result, $\operatorname{dim} H_{0}^{2}\left(\operatorname{sTr}_{D}\right)=\left(\begin{array}{c}n^{\prime} \\ 2\end{array}\right)+n^{\prime}$ since the elements of $\operatorname{sTr}_{D}$ are odd (for an ordinary $n^{\prime}$-dimensional abelian group dim $\left.H_{0}^{2}=\left(\begin{array}{c}n^{\prime} \\ 2\end{array}\right)\right)$. The number $\left(\begin{array}{c}n^{\prime} \\ 2\end{array}\right)+n^{\prime}$ is given nevertheless since it serves as a check on the degrees of freedom: it is equal to the sum of the total real dimensions in the sixth and eigth columns. 
If $Z_{\mu}$ is included separately, this introduces a further extension which requires adding a new LI form associated with it, $\Pi_{\mu}^{(\varphi)}, d \Pi_{\mu}^{(\varphi)}=\frac{1}{2}\left(C \Gamma_{\mu} \mathcal{P}_{+}\right)_{\alpha \beta} d \theta^{\alpha} d \theta^{\beta}$ (we have written the index down for consistency with later notation, as in Sec. 5.1). At the group level this means that the MW translations generate two types of transformations i.e., one has to distinguish between the translations $x^{\mu}$ and the $\varphi_{\mu}$, some of which may be compact, in which case the corresponding group law expression should be understood locally.

\subsubsection{IIA theory centrally extended superspace}

Let us consider now the $H_{\sigma}^{2}\left(\operatorname{sTr}_{10} \circ \operatorname{Spin}(1,9),(\right.$ IIA $\left.)\right)$ spaces. The IIA superalgebra is the $D=10$ algebra associated with two 16-dimensional spinors of opposite chiralities which may be combined into a Majorana spinor. Then (see table), the IIA theory maximally extended algebra [28] is found to be

$$
\begin{aligned}
\left\{D_{\alpha}, D_{\beta}\right\}= & \left(C \Gamma^{\mu}\right)_{\alpha \beta} X_{\mu}+\left(C \Gamma^{\mu_{1} \mu_{2}}\right)_{\alpha \beta} Z_{\mu_{1} \mu_{2}}+\left(C \Gamma^{\mu_{1} \ldots \mu_{5}}\right)_{\alpha \beta} Z_{\mu_{1} \ldots \mu_{5}} \\
& +\left(C \Gamma^{11}\right)_{\alpha \beta} Z+\left(C \Gamma^{\mu} \Gamma^{11}\right)_{\alpha \beta} Z_{\mu}+\left(C \Gamma^{\mu_{1} \ldots \mu_{4}} \Gamma^{11}\right)_{\alpha \beta} Z_{\mu_{1} \ldots \mu_{4}}
\end{aligned}
$$

since the tensor spaces $\left\{\Gamma^{\mu_{1} \ldots \mu_{p}}\right\}$ and $\left\{\Gamma^{\mu_{1} \ldots \mu_{D-p}} \Gamma^{11}\right\}$ are isomorphic by eqn. (2.11). Notice that $X_{\mu}$ and $Z_{\mu}$ belong to different cohomology classes (their corresponding two-cocycles are not cohomologous due to the presence of $\Gamma^{11}$ in the $Z$ term). The associated IIA Lie FDA, involving the LI one-forms dual to the generators in (2.16), is given by

$$
\begin{array}{rlrl}
d \Pi^{\alpha} & =0, & d \Pi^{\mu} & =\frac{1}{2}\left(C \Gamma^{\mu}\right)_{\alpha \beta} \Pi^{\alpha} \Pi^{\beta}, \quad d \Pi_{(z)}^{\mu}=\frac{1}{2}\left(C \Gamma^{\mu} \Gamma^{11}\right)_{\alpha \beta} \Pi^{\alpha} \Pi^{\beta} \\
d \Pi^{\mu_{1} \mu_{2}} & =\frac{1}{2}\left(C \Gamma^{\mu_{1} \mu_{2}}\right)_{\alpha \beta} \Pi^{\alpha} \Pi^{\beta}, \\
d \Pi^{\mu_{1} \ldots \mu_{4}} & =\frac{1}{2}\left(C \Gamma^{\mu_{1} \ldots \mu_{4}} \Gamma^{11}\right)_{\alpha \beta} \Pi^{\alpha} \Pi^{\beta}, & d \Pi^{\mu_{1} \ldots \mu_{5}} & =\frac{1}{2}\left(C \Gamma^{\mu_{1} \ldots \mu_{5}}\right)_{\alpha \beta} \Pi^{\alpha} \Pi^{\beta}, \\
d \Pi & =\frac{1}{2}\left(C \Gamma^{11}\right)_{\alpha \beta} \Pi^{\alpha} \Pi^{\beta} .
\end{array}
$$

The new group parameters define the IIA theory centrally extended superspace, parametrized by the coordinates $\left(\theta^{\alpha}, x^{\mu}, \varphi^{\mu}, \varphi^{\mu_{1} \mu_{2}}, \varphi^{\mu_{1} \ldots \mu_{5}}, \varphi^{\mu_{1} \ldots \mu_{4}}, \varphi\right)$.

The IIB case with $\Pi^{\alpha i} \equiv \mathcal{P}_{+} d \theta^{\alpha i}(i=1,2)$ is treated similarly by noticing that the presence of $\epsilon_{i j}$ allows for $C \Gamma^{\mu_{1} \mu_{2} \mu_{3}} \mathcal{P}_{+}$, which is skew-symmetric.

\section{Non-central extensions and their superspaces}

We start now from standard rigid superspace, eqns. (2.3), (2.4) for real, odd translations. To keep the discussion as general as possible, we rescale $\Pi^{\mu}, \Pi_{\mu_{1} \ldots \mu_{p}}$ by an arbitrary dimensionless constant $a_{s}$, so that (2.4), (2.7) become

$$
d \Pi^{\mu}=a_{s}\left(C \Gamma^{\mu}\right)_{\alpha \beta} \Pi^{\alpha} \Pi^{\beta} \quad, \quad d \Pi_{\mu_{1} \ldots \mu_{p}} \equiv a_{0}\left(C \Gamma_{\mu_{1} \ldots \mu_{p}}\right)_{\alpha \beta} \Pi^{\alpha} \Pi^{\beta} .
$$

Let us fix $p$ and consider the resulting extended superspace, parametrized by $\left(\theta^{\alpha}, x^{\mu}, \varphi_{\mu_{1} \ldots \mu_{p}}\right)$, as our starting group manifold. We look for a non-trivial CE two-cocycle with $p$ indices on the above extended superspace. This may now involve any of the LI forms available, $\Pi^{\mu}, \Pi^{\alpha}$ or $\Pi_{\mu_{1} \ldots \mu_{p}}$. Inspection of the possible Lorentz tensors shows that the external Lorentz indices of this two-cocycle have to be of the type $\left(\mu_{1} \ldots \mu_{p-1} \alpha_{1}\right)$ and, hence, the only available LI two-forms are

$$
\rho_{\mu_{1} \ldots \mu_{p-1} \alpha_{1}}^{(1)}=\left(C \Gamma_{\nu \mu_{1} \ldots \mu_{p-1}}\right)_{\beta \alpha_{1}} \Pi^{\nu} \Pi^{\beta} \quad, \quad \rho_{\mu_{1} \ldots \mu_{p-1} \alpha_{1}}^{(2)}=\left(C \Gamma^{\nu}\right)_{\beta \alpha_{1}} \Pi_{\nu \mu_{1} \ldots \mu_{p-1}} \Pi^{\beta}
$$

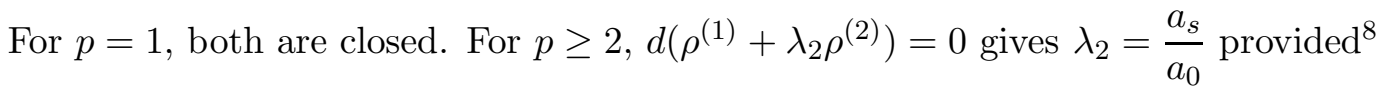

$$
\left(C \Gamma^{\nu}\right)_{\alpha^{\prime} \beta^{\prime}}\left(C \Gamma_{\nu \mu_{1} \ldots \mu_{p-1}}\right)_{\gamma^{\prime} \delta^{\prime}}=0
$$

which holds only for certain values of $(D, p)$ [1] (for $p=1, D=3,4,10$ and, with the appropriate modifications for complex spinors, $D=6$ ). The existence of such a constraint, on both $D$ and $p$,

\footnotetext{
${ }^{8}$ Primed indices are understood to be symmetrised (with unit weight).
} 
is a new feature - the non-triviality of (2.9) only restricted $p$. We introduce now a new one-form $\Pi_{\mu_{1} \ldots \mu_{p-1} \alpha_{1}}$ with

$$
\left.d \Pi_{\mu_{1} \ldots \mu_{p-1} \alpha_{1}}=a_{1}\left(\left(C \Gamma_{\nu \mu_{1} \ldots \mu_{p-1}}\right)_{\beta \alpha_{1}} \Pi^{\nu} \Pi^{\beta}+\frac{a_{s}}{a_{0}}\left(C \Gamma^{\nu}\right)_{\beta \alpha_{1}} \Pi_{\nu \mu_{1} \ldots \mu_{p-1}} \Pi^{\beta}\right)\right)
$$

(for $p=1$ the coefficient of the second term can be arbitrary, see Sec. 5.1).9 The above MC equation implies that both $[D, X]$ and $\left[D, Z^{\mu_{1} \ldots \mu_{p}}\right]$ are modified by a term proportional to $Z^{\mu_{1} \ldots \mu_{p-1} \alpha_{1}}$, the latter being the only central generator at this stage $\left(Z^{\mu_{1} \ldots \mu_{p-1} \alpha_{1}}\right.$ is central because, by construction, $\Pi_{\mu_{1} \ldots \mu_{p-1} \alpha_{1}}$ cannot appear at the r.h.s. of a MC equation expressing the differential of a LI form). This is a general feature of the extension scheme in this section: at any stage in the chain of extensions, the only central generator present is the last one introduced. Thus, each extension is central, but the resulting algebra/group is not a central extension of superspace: all generators but the last one have non-zero commutators as a consequence of the subsequent extensions. A second feature here is that successive extensions substitute one spinorial index for a vectorial one, preserving the total number of indices. The chain ends with the introduction of a generator with $p$ spinorial indices.

Repeating the above procedure, one finds that the next three extensions are in some sense exceptional (see (3.5) below), while the one introducing five spinorial indices and all others after it follow a pattern which can be used to derive a recursion formula. Skipping the somewhat involved algebra (see Appendix A), we list first the results for the next three extensions

$$
\begin{aligned}
& d \Pi_{\mu_{1} \ldots \mu_{p-2} \alpha_{1} \alpha_{2}}=a_{2}\left(\left(C \Gamma_{\nu \rho \mu_{1} \ldots \mu_{p-2}}\right)_{\alpha_{1} \alpha_{2}} \Pi^{\nu} \Pi^{\rho}+\frac{a_{s}}{a_{0}}\left(C \Gamma^{\nu}\right)_{\alpha_{1} \alpha_{2}} \Pi_{\nu \rho \mu_{1} \ldots \mu_{p-2}} \Pi^{\rho}\right. \\
& \left.-\frac{a_{s}}{a_{1}}\left(C \Gamma^{\nu}\right)_{\alpha_{1} \alpha_{2}} \Pi_{\nu \mu_{1} \ldots \mu_{p-2} \beta} \Pi^{\beta}-8 \frac{a_{s}}{a_{1}}\left(C \Gamma^{\nu}\right)_{\alpha_{1}^{\prime} \beta} \Pi_{\nu \mu_{1} \ldots \mu_{p-2} \alpha_{2}^{\prime}} \Pi^{\beta}\right), \\
& d \Pi_{\mu_{1} \ldots \mu_{p-3} \alpha_{1} \alpha_{2} \alpha_{3}}=a_{3}\left(\left(C \Gamma^{\nu}\right)_{\alpha_{1}^{\prime} \alpha_{2}^{\prime}} \Pi_{\nu \rho \mu_{1} \ldots \mu_{p-3} \alpha_{3}^{\prime}} \Pi^{\rho}+\frac{5 a_{1}}{4 a_{2}}\left(C \Gamma^{\nu}\right)_{\alpha_{1}^{\prime} \beta} \Pi_{\nu \mu_{1} \ldots \mu_{p-3} \alpha_{2}^{\prime} \alpha_{3}^{\prime}} \Pi^{\beta}\right. \\
& \left.+\frac{a_{1}}{4 a_{2}}\left(C \Gamma^{\nu}\right)_{\alpha_{1}^{\prime} \alpha_{2}^{\prime}} \Pi_{\nu \mu_{1} \ldots \mu_{p-3} \beta \alpha_{3}^{\prime}} \Pi^{\beta}\right) \\
& d \Pi_{\mu_{1} \ldots \mu_{p-4} \alpha_{1} \alpha_{2} \alpha_{3} \alpha_{4}}=a_{4}\left(\left(C \Gamma^{\nu}\right)_{\alpha_{1}^{\prime} \alpha_{2}^{\prime}} \Pi_{\nu \rho \mu_{1} \ldots \mu_{p-4} \alpha_{3}^{\prime} \alpha_{4}^{\prime}} \Pi^{\rho}-\frac{48 a_{s} a_{2}}{5 a_{1} a_{3}}\left(C \Gamma^{\nu}\right)_{\alpha_{1}^{\prime} \beta} \Pi_{\nu \mu_{1} \ldots \mu_{p-4} \alpha_{2}^{\prime} \alpha_{3}^{\prime} \alpha_{4}^{\prime}} \Pi^{\beta}\right. \\
& \left.-\frac{12 a_{s} a_{2}}{5 a_{1} a_{3}}\left(C \Gamma^{\nu}\right)_{\alpha_{1}^{\prime} \alpha_{2}^{\prime}} \Pi_{\nu \mu_{1} \ldots \mu_{p-4} \beta \alpha_{3}^{\prime} \alpha_{4}^{\prime}} \Pi^{\beta}\right)
\end{aligned}
$$

(the $a_{k}$ 's in the r.h.s. normalise the П's with $k$ spinorial indices). For the remaining extensions, which introduce one-forms with five or more spinorial indices, one establishes the following recursion formula

$$
\begin{aligned}
d \Pi_{\mu_{1} \ldots \mu_{p-(k+2)} \alpha_{1} \ldots \alpha_{k+2}}= & a_{k+2}\left\{\left(C \Gamma^{\nu}\right)_{\alpha_{1}^{\prime} \alpha_{2}^{\prime}} \Pi_{\nu \rho \mu_{1} \ldots \mu_{p-(k+2)} \alpha_{3}^{\prime} \ldots \alpha_{k+2}^{\prime}} \Pi^{\rho}\right. \\
& +\lambda_{2}^{(k+2)}\left(C \Gamma^{\nu}\right)_{\alpha_{1}^{\prime} \beta} \Pi_{\nu \mu_{1} \ldots \mu_{p-(k+2)} \alpha_{2}^{\prime} \ldots \alpha_{k+2}^{\prime}} \Pi^{\beta} \\
& \left.+\lambda_{3}^{(k+2)}\left(C \Gamma^{\nu}\right)_{\alpha_{1}^{\prime} \alpha_{2}^{\prime}} \Pi_{\nu \mu_{1} \ldots \mu_{p-(k+2)}} \beta \alpha_{3}^{\prime} \ldots \alpha_{k+2}^{\prime} \Pi^{\beta}\right\},
\end{aligned}
$$

where

$$
\lambda_{2}^{(k+2)}=-\frac{a_{s}}{a_{k+1}}\left(\frac{2}{\lambda_{2}^{(k+1)}}+\frac{k}{\lambda_{3}^{(k+1)}}\right) \quad, \quad \lambda_{3}^{(k+2)}=-\frac{a_{s}}{a_{k+1}} \frac{k+1}{\lambda_{2}^{(k+1)}} .
$$

Notice that the above recursion starts at $k=3$, which implies $p \geq 5$. On the other hand, the maximum value of $p$ (of interest to us) for which (3.3) holds true is $p=5$, i.e. (3.6) is relevant here only for $k=3, p=5$. It is easily checked that $\left[\Pi_{\mu_{1} \ldots \mu_{p-l} \alpha_{1} \ldots \alpha_{l}}\right]=L^{1+\frac{l}{2}}$. We give related explicit results, for $p=1,2$, in Sec. 5 .

\footnotetext{
${ }^{9}$ For $p=1$, the one-form in the l.h.s. of (3.4) becomes $\Pi_{\alpha}$ - notice that this is unrelated to $\Pi^{\alpha}$ (so that e.g. $d \Pi_{\alpha}$ is non-zero). In general, we will not raise or lower the Lorentz indices of forms, their position being used to distinguish between different types of them as in [19].
} 


\section{Structure of the new superspaces and Noether currents}

\subsection{Fibre bundle structure}

All extended superspaces have a natural bundle structure, in which the basis is the group to be extended and the fibre is the group by which we extend. For instance, superspace $\Sigma$ itself and the various extensions $\tilde{\Sigma}$ in Sec. 2.2 may be considered as the total spaces of principal bundles over the $\mathrm{sTr}_{D}$ 's of the specific theory. The two-forms which define the extensions are curvatures of invariant connections valued on the central algebras by which $\mathrm{sTr}_{D}$ is extended. The $D$ 's are then the horizontal lifts of the vector fields $\frac{\partial}{\partial \theta^{\alpha}}$ on the specific base manifold $\operatorname{sTr}_{D}$; this justifies the 'covariant derivative' name which may be given to the $D$ 's in the algebra of the $\tilde{\Sigma}$ 's. Similar considerations apply at any step in the chain of extensions in Sec. 3. From this point of view, after the last step, one has a bundle structure with the last coordinate in the fibre and all the rest in the base. As we show below, there is also another relevant bundle structure with $\Sigma$ in the base and all new coordinates in the fibre.

Let us now discuss the general case treated in Sec. 3. $\tilde{\Sigma}$ is parametrised by the coordinates $\left(\theta^{\alpha}, x^{\mu}, \varphi_{\mu_{1} \ldots \mu_{p}}, \varphi_{\mu_{1} \ldots \mu_{p-1} \alpha_{1}}, \ldots, \varphi_{\alpha_{1} \ldots \alpha_{p}}\right)$. We will denote them collectively by the row vector $\phi=$ $\left(z^{a}, \varphi_{A}\right)$ where $z$ parametrizes the base (superspace $\Sigma$ ) and $\varphi$ the fibre (the space of all new coordinates). Referring to this block form, we will say that the superspace part is 'two-dimensional' while the fibre part has 'dimension' $p+1$. The LI one-forms that reduce to the differentials of these coordinates at the identity will be denoted by $\Pi=\left(H^{a}, \Theta_{A}\right)$ and the dual LI vector fields by the column vector $Z=\left(D_{a}, Y^{A}\right)^{t}(t$ denotes matrix transposition). The corresponding RI objects will carry an additional hat.

Under a right group transformation, $g \rightarrow g g^{\prime}, Z$ transforms like $Z \rightarrow T^{\prime t} Z, T^{\prime}$ being a matrix of (primed) functions on the group, called the adjoint representation. It holds

$$
T^{\prime \prime}=T^{\prime} T \quad,\left.\quad Z \cdot T\right|_{e}=\rho_{\mathrm{adj}}(Z),
$$

$\rho_{\mathrm{adj}}(Z)$ being the adjoint representation of $Z$, given by the structure constants. Inspection of the MC equations then reveals that $T$ is a lower triangular matrix with units along the diagonal. We put accordingly

$$
T^{t}=\left(\begin{array}{cc}
A & C \\
0 & B
\end{array}\right) \quad, \quad\left(T^{t}\right)^{-1}=\left(\begin{array}{cc}
A^{-1} & -A^{-1} C B^{-1} \\
0 & B^{-1}
\end{array}\right)
$$

with $A, B$ upper triangular matrices. The dimensions of $A, B, C$ (in block form) are $2 \times 2,(p+1) \times$ $(p+1), 2 \times(p+1)$ respectively. (4.2) shows that the fibre is a subgroup, with adjoint representation given by $B^{t}$. In this notation, the LI vector fields transform like

$$
\left(\begin{array}{c}
D \\
Y
\end{array}\right) \rightarrow\left(\begin{array}{c}
A^{\prime} D+C^{\prime} Y \\
B^{\prime} Y
\end{array}\right)
$$

The LI forms similarly transform according to $\Pi \rightarrow \Pi\left(T^{\prime t}\right)^{-1}$, i.e.

$$
\left(\begin{array}{ll}
H, & \Theta
\end{array}\right) \rightarrow\left(\begin{array}{lll}
H A^{\prime-1}, & -H A^{\prime-1} C^{\prime} B^{\prime-1}+\Theta B^{\prime-1}
\end{array}\right) .
$$

For the RI objects it holds $\hat{Z}=\left(T^{t}\right)^{-1} Z, \hat{\Pi}=\Pi T^{t}$ i.e.

$$
\left(\begin{array}{c}
\hat{D} \\
\hat{Y}
\end{array}\right)=\left(\begin{array}{c}
A^{-1} D-A^{-1} C B^{-1} Y \\
B^{-1} Y
\end{array}\right) \quad, \quad(\hat{H}, \quad \hat{\Theta})=\left(\begin{array}{cc}
H A, & H C+\Theta B
\end{array}\right) .
$$

The Lie algebra valued one-form $\omega=\Theta Y$ serves as a connection in the bundle. Indeed, one easily verifies that $\omega$ is invariant under (4.3), (4.4) when $T$ is restricted to the subgroup of the fibre $(A=I$, $C=0$ ). The horizontal subspace is spanned by the kernel of $\omega$, i.e. by the components of $D$, the latter being the horizontal lifts of the standard superspace generators $D_{\alpha}^{(s)}=\frac{\partial}{\partial \theta^{\alpha}}, X_{\mu}^{(s)}=\frac{\partial}{\partial x^{\mu}}+$ $\frac{1}{2}\left(C \Gamma_{\mu}\right)_{\alpha \beta} \theta^{\beta} \frac{\partial}{\partial \theta^{\alpha}}$.

In later applications, in section 5, the explicit form of the matrix $B^{-1}$ is needed - we present here a few remarks that facilitate its computation. Inspection of the r.h.s. of the $\mathrm{MC}$ equations for the new one-forms, in section 3 , shows that they always contain one new one-form, multiplied by a $\Pi^{\alpha}$ or $\Pi^{\mu}$. 
For the dual Lie algebra this implies that the new generators commute among themselves and only have, in general, non-zero commutators with the superspace generators $D_{\alpha}, X_{\mu}$. In other words, the group by which we extend $\Sigma$ is abelian (to begin with) and its generators acquire, as a result of the extension, non-zero commutators only with the superspace generators. The structure of the resulting Lie algebra is, in symbolic form,

$$
[D, D] \sim D+Y \quad, \quad[D, Y] \sim Y \quad, \quad[Y, Y]=0
$$

in the notation introduced earlier. For $T$, the expression

$$
T=e^{\phi^{A} \rho_{a d j}\left(Z_{A}\right)} \equiv e^{z^{a} \rho_{a d j}\left(D_{a}\right)+\varphi_{A} \rho_{a d j}\left(Y^{A}\right)}
$$

is well known. From the third of (4.6) though, we infer that the restriction of $\rho_{\text {adj }}(Y)$ to the fibre (i.e. to the sub-block corresponding to $\left.B^{t}\right)$ is zero. Denoting this sub-block by $\rho_{a d j}^{(f)}(Y)$ we find for $B$

$$
B=e^{z^{a} \rho_{a d j}^{(f)}\left(D_{a}\right)^{t}} \equiv e^{\theta^{\alpha} \rho_{a d j}^{(f)}\left(D_{\alpha}\right)^{t}+x^{\mu} \rho_{a d j}^{(f)}\left(X_{\mu}\right)^{t}}
$$

where the matrices $\rho_{a d j}^{(f)}\left(D_{a}\right)$ are given by the structure constants that appear in (the explicit form of) the second of (4.6). The interesting point here is that $B$ depends on $(\theta, x)$ only - the new variables enter in $T$ only through $A, C$.

\subsection{Invariant actions for the minimal branescan}

As already mentioned, part of the motivation for studying superspace extensions comes from their relevance in the construction of manifestly invariant $p$-brane actions. For the branes of the old branescan, WZ terms on $\Sigma$ have the form

$$
S_{W Z}=\int_{W} d^{p+1} \xi \mathcal{L}_{W Z}=\lambda \int_{W} \phi^{*}(b)
$$

where $b$ is defined $\mathbb{T}$ as the potential of the closed $(p+2)$-form $h$ on superspace

$$
h=\left(C \Gamma_{\mu_{1} \ldots \mu_{p}}\right)_{\alpha \beta} \Pi^{\mu_{1}} \ldots \Pi^{\mu_{p}} \Pi^{\alpha} \Pi^{\beta} \quad, \quad d b=h \quad .
$$

$W$ in (4.9) is the $(p+1)$-dimensional worldvolume swept out by the $p$-brane, parametrized by $\left\{\xi^{i}\right\}=$ $\left(\tau, \sigma^{1}, \ldots, \sigma^{p}\right), i=0,1 \ldots p$ and $\phi^{*}$ is the pullback of the embedding $\phi: W \rightarrow \Sigma$. The constant $\lambda$ is fixed by the requirement of $\kappa$-invariance of the total action [1] (we will ignore $\lambda$ henceforth). As is well known [1] (see also [2]), the closure of $h$ is equivalent to the condition (3.3) which we have seen to guarantee the existence of the non-central extensions of Sec. 3. Using the new LI one-forms available we may obtain a LI potential $\tilde{b}$ for $h$ on $\tilde{\Sigma}$. Its general form is

$$
\tilde{b}=\sum_{k=0}^{p} \Pi_{\mu_{1} \ldots \mu_{p-k} \alpha_{1} \ldots \alpha_{k}}\left(b_{k} \Pi^{\mu_{1}} \ldots \Pi^{\mu_{p-k}} \Pi^{\alpha_{1}} \ldots \Pi^{\alpha_{k}}\right) \equiv \Theta_{C} \Lambda^{C}
$$

where the last equation uses the notation of the previous section and defines $\Lambda^{C}$. The $b_{k}$ 's are numerical constants, determined by the second eqn. in (4.10). We check that $[\tilde{b}]=L^{1+\frac{k}{2}} L^{p-k} L^{\frac{k}{2}}=L^{p+1}$.

We compute now the explicit form for an invariant $\mathcal{L}_{W Z}$ in (4.9) (i.e., with $\tilde{b}$ instead of $b$ ). For a general one-form $\Pi$ we put $\phi^{*}(\Pi) \equiv \Pi_{i} d \xi^{i}$, so that

$$
\Pi_{i}^{\alpha}=\partial_{i} \theta^{\alpha}, \quad \Pi_{i}^{\mu}=\partial_{i} x^{\mu}+a_{s}\left(C \Gamma^{\mu}\right)_{\alpha \beta} \theta^{\alpha} \partial_{i} \theta^{\beta} .
$$

Eqns. (4.11) and (4.9) give for the Lagrangian

$$
\mathcal{L}_{W Z}=\Theta_{C i} \Lambda^{C i}
$$

where, expanding multi-indices and using the above notation,

$$
\Lambda^{\mu_{1} \ldots \mu_{p-k} \alpha_{1} \ldots \alpha_{k} i} \equiv b_{k} \epsilon^{i j_{1} \ldots j_{p}} \Pi_{j_{1}}^{\mu_{1}} \ldots \Pi_{j_{p-k}}^{\mu_{p-k}} \Pi_{j_{p-k+1}}^{\alpha_{1}} \ldots \Pi_{j_{p}}^{\alpha_{k}}
$$

${ }^{10}$ For an explicit form of the quasi-invariant $b$ on $\Sigma$ see 447 . 


\subsection{Noether currents for the new symmetries}

The invariance under translations of the action of the supersymmetric objects implies the existence of conserved currents. The integrals of the charge densities over a spacelike section of the worldvolume give constants of the motion for the $p$-brane. When the action contains the standard WZ term $b$, the Noether current includes a term $\Delta$ coming from the quasi-invariance of $b, \delta b=d \Delta$. This was used in [12] to find topological extensions of the supersymmetry algebra. When $b$ is replaced by the invariant $b$ in (4.11), $\Delta$ is no longer present. However, the Noether current receives now a contribution from the additional fields $\phi^{*}\left(\varphi_{A}\right)$, which leads to the same result.

One can derive general expressions for the currents associated with the new generators. In the present case, where the relevant part of the Lagrangian is obtained by pulling back to $W$ forms initially defined on $\tilde{\Sigma}$, it is convenient to work on the extended superspace, where quantities have a direct geometrical interpretation, and to pull the result back to $W$ at the end. To keep the discussion general, consider a manifold $M$ which can serve as worldvolume and a target space $N$, of dimensions $m, n$ respectively $(m<n)$ and an embedding $\phi$ of $M$ into $N, \phi: x \mapsto y(x)$ where $\left\{x^{i}\right\}\left(\left\{y^{j}\right\}\right)$ are local coordinates on $M(N)$. Consider furthermore an action $S$ given by

$$
S=\int_{M} \phi^{*}(\alpha)
$$

where $\alpha$ is a $k$-form on $N$ and $\phi^{*}$ is the pullback map associated with the embedding. We assume that the submanifolds $x^{0}=x_{\text {init }}^{0}, x^{0}=x_{\text {fin }}^{0}$ of $M$ form its boundary $\partial M$ - their embeddings in $N$ are the initial and final configuration respectively. The equations of motion are

$$
\delta_{Y} S=\int_{M} \phi^{*}\left(L_{Y} \alpha\right)=0
$$

where $Y$ is an arbitrary vector field on $N$ which vanishes on $\phi(\partial M)$ - we denote their solutions generically by $\phi_{c l}$. Proceeding along the lines of the standard derivation, with inner derivations taking up the role of the partials $\frac{\partial}{\partial \phi_{, i}}$, one finds the equations of motion in the form

$$
\phi^{*}\left(i_{Y} d \alpha\right)=0
$$

where now $Y$ is an arbitrary vector field, not necessarily vanishing on $\partial M$. For a symmetry generated by $Y_{0}$ one obtains

$$
d\left(\phi_{c l}^{*}\left(J_{\left(Y_{0}\right)}\right)\right)=0, \quad J_{\left(Y_{0}\right)} \equiv i_{Y_{0}} \alpha,
$$

which is the current conservation equation. For a quasi-invariant Lagrangian, $\phi^{*}\left(L_{Y_{0}} \alpha\right)=\phi^{*}(d \Delta)$, the conserved current picks up a term in $\Delta, \phi^{*}\left(J_{\left(Y_{0}\right)}\right)=\phi^{*}\left(i_{Y_{0}} \alpha-\Delta\right)$.

In the present case, $(M, N, \alpha)$ correspond to $(W, \tilde{\Sigma}, \tilde{b})$. The variation of the total action from that of the new coordinates comes only from $\mathcal{L}_{W Z}$ so that (4.17) gives

$$
0=\phi^{*}\left(i_{Y} d \tilde{b}\right)=\phi^{*}\left(i_{Y} h\right)
$$

where $Y$ is an arbitrary vector field along the fibre. Since $h$ is horizontal, the above equations of motion obtained from variations of the $\varphi_{A}$ 's, are satisfied trivially, consistent with the appearence of the $\varphi_{A}$ 's in the Lagrangian through exact differentials. For the Noether currents associated to translations along the new coordinates we have $Y_{0} \rightarrow \hat{Y}^{A}$ and (4.18) gives

$$
d\left(\phi_{c l}^{*}\left(J^{A}\right)\right)=0 \quad, \quad J^{A}=i_{\hat{Y}^{A}} \tilde{b}
$$

With $\tilde{b}$ as in (4.11) and $\hat{Y}^{A}=\left(B^{-1}\right)^{A}{ }_{C} Y^{C}$ (see (4.5)), the second of (4.20) gives for $J^{A}$

$$
J^{A}=\left(B^{-1}\right)^{A}{ }_{D} i_{Y^{D}} \Pi_{C} \Lambda^{C}=\left(B^{-1}\right)^{A}{ }_{C} \Lambda^{C},
$$

since $i_{Y^{D}} \Pi_{C}=\delta^{D}{ }_{C}$. Notice that $J^{A}$ is, in this case, a form on $\Sigma$ (rather than $\tilde{\Sigma}$ ). Effecting explicitly the pullback in the first of $(4.20)$ we find $\square$

$$
\partial_{i} j^{A i}=0 \quad, \quad j^{A i}(\xi) \equiv\left(B^{-1}\right)_{C}^{A}(\xi) \Lambda^{C i}(\xi) \quad .
$$

\footnotetext{
${ }^{11}$ Eqn. (4.22) for $j^{A i}$ also follows from the standard expression for the current associated with an 'internal' symmetry of a Lagrangian $\mathcal{L}, j^{A i}=\delta^{A} \varphi(\xi) \frac{\partial \mathcal{L}}{\partial_{i} \varphi(\xi)}$. However, for the currents considered here the relevant part of $\mathcal{L}$ is just $\mathcal{L}_{W Z}$. Since $\mathcal{L}_{W Z}$ is obtained from a form on $\tilde{\Sigma}$, the above derivation allows us to exploit the geometry of $\tilde{\Sigma}$. The above expression for the current also makes clear that, within the canonical formalism, the integrated charge operators will reproduce the original symmetry algebra.
} 
Finally, the conserved charges $Q^{A}$ are given by (expanding multi-indices)

$$
Q^{\mu_{1} \ldots \mu_{p-k} \alpha_{1} \ldots \alpha_{k}}=\int_{W_{\tau}} d \sigma^{1} \ldots d \sigma^{p} \sum_{m=0}^{p}\left(B^{-1}\right)_{\substack{\nu_{1} \ldots \nu_{p-m} \beta_{1} \ldots \beta_{m} \\ \mu_{1} \ldots \mu_{p-k} \alpha_{1} \ldots \alpha_{k}}} b^{0 j_{1} \ldots j_{p}} \Pi_{j_{1}}^{\nu_{1}} \ldots \Pi_{j_{p-m}}^{\nu_{p-m}} \Pi_{j_{p-m+1}}^{\beta_{1}} \ldots \Pi_{j_{p}}^{\beta_{m}}
$$

where $W_{\tau}$ is a hypersurface of constant $\tau$. Notice that since $B=B(\theta, x)$, the integrand above involves only superspace variables.

\section{Applications: $p=1,2$}

\section{1 $D=10, \mathbf{N}=1$ and the Green-Schwarz superstring}

The case of the superstring is somewhat special, from the point of view of the extension algorithm of Sec. 3: the first additional generator to be introduced, $Z^{\mu}$, is a vector, as $X_{\mu}$. We shall keep it here separate and denote by $\varphi_{\mu}$ the associated parameter. Fixing $\left(a_{s}, a_{0}, a_{1}\right)=\left(\frac{1}{2}, \frac{1}{2}, 1\right)$ in $(3.1),(3.4)$, we find for the FDA

$$
\begin{aligned}
d \Pi^{\alpha} & =0, & d \Pi^{\mu} & =\frac{1}{2}\left(C \Gamma^{\mu}\right)_{\alpha \beta} \Pi^{\alpha} \Pi^{\beta}, \\
d \Pi_{\alpha} & =\left(C \Gamma_{\mu}\right)_{\alpha \beta} \Pi^{\mu} \Pi^{\beta}+\left(C \Gamma^{\mu}\right)_{\alpha \beta} \Pi_{\mu} \Pi^{\beta}, & d \Pi_{\mu}^{(\varphi)} & =\frac{1}{2}\left(C \Gamma_{\mu}\right)_{\alpha \beta} \Pi^{\alpha} \Pi^{\beta},
\end{aligned}
$$

where $\mu=0, \ldots, 9$. Notice that $d\left(d \Pi_{\alpha}\right)=0$ is implied by (3.3) for $p=1,\left(C \Gamma^{\mu}\right)_{\alpha^{\prime} \beta^{\prime}}\left(C \Gamma_{\mu}\right)_{\gamma^{\prime} \delta^{\prime}}=0$. $\Pi_{\mu}^{(\varphi)}$ in the above equation is the one obtained from the second of (3.1) for $p=1$ - we have added a superscript to avoid any confusion with $\Pi^{\mu}$, since they have similar differentials; recall also that $\Pi_{\alpha}$ and $\Pi^{\alpha}$ are unrelated. As mentioned in Sec. 3, the two terms in the r.h.s. of the last of (5.1) are individually closed and hence their relative normalization cannot be fixed by requiring $d\left(d \Pi_{\alpha}\right)=0$. We have nevertheless chosen the above symmetric normalization for convenience - the results that follow, and in particular (5.12) that involves cancellations, do not depend essentially on this choice.

The corresponding Lie algebra is given by

$$
\left\{D_{\alpha}, D_{\beta}\right\}=\left(C \Gamma^{\mu}\right)_{\alpha \beta} X_{\mu}+\left(C \Gamma_{\mu}\right)_{\alpha \beta} Z^{\mu}, \quad\left[D_{\alpha}, X_{\mu}\right]=\left(C \Gamma_{\mu}\right)_{\alpha \beta} Z^{\beta}, \quad\left[D_{\alpha}, Z^{\mu}\right]=\left(C \Gamma^{\mu}\right)_{\alpha \beta} Z^{\beta},
$$

which reduces to the Green algebra [13] if one omits $Z^{\mu}$. The associated group manifold (extended superspace) $\tilde{\Sigma}$ is parametrized by $\left(\theta^{\alpha}, x^{\mu}, \varphi_{\mu}, \varphi_{\alpha}\right)$ via

$$
g\left(\theta^{\alpha}, x^{\mu}, \varphi_{\mu}, \varphi_{\alpha}\right)=e^{\theta^{\alpha} D_{\alpha}+x^{\mu} X_{\mu}+\varphi_{\mu} Z^{\mu}+\varphi_{\alpha} Z^{\alpha}} .
$$

Making use of the Baker-Cambell-Hausdorff (BCH) formula, where, for the algebra (5.2), terms of order four and higher vanish, we find the $\tilde{\Sigma}$ group law

$$
\begin{array}{rlrl}
\theta^{\prime \prime \alpha}= & \theta^{\prime \alpha}+\theta^{\alpha}, & x^{\prime \prime \mu} & =x^{\prime \mu}+x^{\mu}-\frac{1}{2}\left(C \Gamma^{\mu}\right)_{\alpha \beta} \theta^{\prime \alpha} \theta^{\beta} \\
\varphi_{\alpha}^{\prime \prime}= & \varphi_{\alpha}^{\prime}+\varphi_{\alpha}+\frac{1}{2}\left(C \Gamma_{\mu}\right)_{\alpha \beta} \theta^{\prime \beta} x^{\mu}-\frac{1}{2}\left(C \Gamma_{\mu}\right)_{\alpha \beta} x^{\prime \mu} \theta^{\beta} & \varphi_{\mu}^{\prime \prime}=\varphi_{\mu}^{\prime}+\varphi_{\mu}-\frac{1}{2}\left(C \Gamma_{\mu}\right)_{\alpha \beta} \theta^{\alpha} \theta^{\beta} . \\
& +\frac{1}{2}\left(C \Gamma^{\mu}\right)_{\alpha \beta} \theta^{\prime \beta} \varphi_{\mu}-\frac{1}{2}\left(C \Gamma^{\mu}\right)_{\alpha \beta} \varphi_{\mu}^{\prime} \theta^{\beta} & \\
& +\frac{1}{6}\left(C \Gamma_{\mu}\right)_{\alpha \beta}\left(C \Gamma^{\mu}\right)_{\gamma \delta}\left(\theta^{\prime \gamma} \theta^{\prime \beta} \theta^{\delta}+\theta^{\prime \delta} \theta^{\gamma} \theta^{\beta}\right), & &
\end{array}
$$

The bilinear terms in the expression for $\varphi_{\alpha}^{\prime \prime}$ are the ones that give rise to the fourth of the MC equations (5.1) - the trilinear terms are required by the associativity of the group law. Their sum gives the spinor valued two-cocycle $\xi_{\alpha}$ associated with the central extension of $\tilde{\Sigma}\left(\theta^{\alpha}, x^{\mu}, \varphi_{\mu}\right)$ by $\varphi_{\alpha}$.

\footnotetext{
${ }^{12}$ In Sec. 5.1 all spinors are Majorana-Weyl $\left(\theta^{\alpha} \equiv\left(\mathcal{P}_{+} \theta\right)^{\alpha}\right.$, etc. $)$.
} 
One can now relate the LI one-forms to the coordinate differentials

$$
\begin{array}{rlrl}
\Pi^{\alpha}= & d \theta^{\alpha}, & \Pi^{\mu}=d x^{\mu}+\frac{1}{2}\left(C \Gamma^{\mu}\right)_{\alpha \beta} \theta^{\alpha} d \theta^{\beta} \\
\Pi_{\alpha}= & d \varphi_{\alpha}-\frac{1}{2}\left(C \Gamma_{\mu}\right)_{\alpha \beta} \theta^{\beta} d x^{\mu}-\frac{1}{2}\left(C \Gamma^{\mu}\right)_{\alpha \beta} \theta^{\beta} d \varphi_{\mu} & \Pi_{\mu}^{(\varphi)}=d \varphi_{\mu}+\frac{1}{2}\left(C \Gamma_{\mu}\right)_{\alpha \beta} \theta^{\alpha} . \\
& +\frac{1}{2}\left(C \Gamma_{\mu}\right)_{\alpha \beta} x^{\mu} d \theta^{\beta}+\frac{1}{2}\left(C \Gamma^{\mu}\right)_{\alpha \beta} \varphi_{\mu} d \theta^{\beta} & & \\
& +\frac{1}{3}\left(C \Gamma_{\mu}\right)_{\alpha \beta}\left(C \Gamma^{\mu}\right)_{\gamma \delta} \theta^{\gamma}, & &
\end{array}
$$

(see also [20] although, omitting $\Pi_{\mu}^{(\varphi)}$, we disagree with the corresponding expressions there). One may check that the LI forms in (5.5) satisfy the FDA (5.1). From the group composition law, one can compute the LI vector fields dual to (5.5) satisfying (5.2)

$$
\begin{aligned}
D_{\alpha}= & \partial_{\alpha}+\frac{1}{2}\left(C \Gamma^{\mu}\right)_{\alpha \beta} \theta^{\beta} \partial_{\mu}+\frac{1}{2}\left(C \Gamma_{\mu}\right)_{\alpha \beta} \theta^{\beta} \partial^{\mu} \\
& -\frac{1}{2}\left(C \Gamma_{\mu}\right)_{\alpha \beta} x^{\mu} \partial^{\beta}-\frac{1}{2}\left(C \Gamma^{\mu}\right)_{\alpha \beta} \varphi_{\mu} \partial^{\beta}+\frac{1}{6}\left(C \Gamma^{\mu}\right)_{\alpha \beta}\left(C \Gamma_{\mu}\right)_{\gamma \delta} \theta^{\beta} \theta^{\delta} \partial^{\gamma} \\
X_{\mu}= & \partial_{\mu}+\frac{1}{2}\left(C \Gamma_{\mu}\right)_{\alpha \beta} \theta^{\beta} \partial^{\alpha} \\
Z^{\mu}= & \partial^{\mu}+\frac{1}{2}\left(C \Gamma^{\mu}\right)_{\alpha \beta} \theta^{\beta} \partial^{\alpha} \\
Z^{\alpha}= & \partial^{\alpha} \quad\left(\text { note } \partial^{\mu} \equiv \frac{\partial}{\partial \varphi_{\mu}}, \partial^{\alpha} \equiv \frac{\partial}{\partial \varphi_{\alpha}}\right) .
\end{aligned}
$$

Effecting a right group translation in (5.5) and reexpressing the result in terms of the $\Pi$ 's, or computing the exponential in (4.7), one finds for $\left(T^{t}\right)^{-1}$

$$
\left(T^{t}\right)^{-1}=\left(\begin{array}{cc|cc}
\delta_{\gamma}{ }^{\alpha} & -\left(C \Gamma^{\mu}\right)_{\gamma \delta} \theta^{\delta} & -\left(C \Gamma_{\kappa}\right)_{\gamma \delta} \theta^{\delta} & \left(C \Gamma_{\lambda}\right)_{\gamma \beta} x^{\lambda}+\left(C \Gamma^{\nu}\right)_{\gamma \beta} \varphi_{\nu}+\left(C \Gamma_{\lambda}\right)_{\beta \delta}\left(C \Gamma^{\lambda}\right)_{\gamma \epsilon} \theta^{\epsilon} \theta^{\delta} \\
0 & \delta_{\nu}{ }^{\mu} & 0 & -\left(C \Gamma_{\nu}\right)_{\beta \delta} \theta^{\delta} \\
\hline 0 & 0 & \delta^{\rho}{ }_{\kappa} & -\left(C \Gamma^{\rho}\right)_{\beta \delta} \theta^{\delta} \\
0 & 0 & 0 & \delta^{\zeta} \beta
\end{array}\right)
$$

(the matrix indices are $\gamma \nu \rho \zeta$ for the rows and $\alpha \mu_{\kappa \beta}$ for the columns). When eqns. (5.4) are linearized in the primed variables (viewed as the parameters of the transformation), they provide a realization of the algebra (5.2), acting on the coordinate (unprimed) variables. For our particular choice of parametrization (5.3), this action is rendered non-linear by the last term in the expression for $\varphi_{\alpha}^{\prime \prime}$. This term can be eliminated by modifying the two-cocycle by a two-coboundary, the latter being generated by a suitable spinor-valued function $\eta_{\alpha}$ on $\tilde{\Sigma}\left(\theta^{\alpha}, x^{\mu}, \varphi_{\mu}\right)$. Indeed, with $\eta_{\alpha} \equiv \frac{1}{6}\left(C \Gamma_{\mu}\right)_{\alpha \beta} x^{\mu} \theta^{\beta}+$ $\frac{1}{6}\left(C \Gamma^{\mu}\right)_{\alpha \beta} \varphi_{\mu} \theta^{\beta}$ we find for the coboundary $\xi_{\alpha}^{c o b}\left(g^{\prime}, g\right) \equiv \eta_{\alpha}\left(g^{\prime} g\right)-\eta_{\alpha}\left(g^{\prime}\right)-\eta_{\alpha}(g)$, the expression

$$
\begin{aligned}
\xi_{\alpha}^{c o b}= & \frac{1}{6}\left(C \Gamma_{\mu}\right)_{\alpha \beta}\left(x^{\prime \mu} \theta^{\beta}+\theta^{\prime \beta} x^{\mu}\right)+\frac{1}{6}\left(C \Gamma^{\mu}\right)_{\alpha \beta}\left(\varphi_{\mu}^{\prime} \theta^{\beta}+\theta^{\prime \beta} \varphi_{\mu}\right) \\
& +\frac{1}{6}\left(C \Gamma_{\mu}\right)_{\alpha \beta}\left(C \Gamma^{\mu}\right)_{\gamma \delta}\left(\theta^{\prime \gamma} \theta^{\prime \beta} \theta^{\delta}-\theta^{\prime \delta} \theta^{\gamma} \theta^{\beta}\right) .
\end{aligned}
$$

The new cocycle $\bar{\xi}_{\alpha}=\xi_{\alpha}+\xi_{\alpha}^{c o b}$ modifies the last eqn. in (5.4) to read

$$
\begin{aligned}
\bar{\varphi}_{\alpha}^{\prime \prime}= & \bar{\varphi}_{\alpha}^{\prime}+\bar{\varphi}_{\alpha}+\left(C \Gamma_{\mu}\right)_{\alpha \beta}\left(\frac{2}{3} \theta^{\prime \beta} x^{\mu}-\frac{1}{3} x^{\prime \mu} \theta^{\beta}\right)+\left(C \Gamma^{\mu}\right)_{\alpha \beta}\left(\frac{2}{3} \theta^{\prime \beta} \varphi_{\mu}-\frac{1}{3} \varphi_{\mu}^{\prime} \theta^{\beta}\right) \\
& +\frac{1}{3}\left(C \Gamma_{\mu}\right)_{\alpha \beta}\left(C \Gamma^{\mu}\right)_{\gamma \delta} \theta^{\prime \gamma} \theta^{\prime \beta} \theta^{\delta}
\end{aligned}
$$

which is linear in the unprimed variables although the definite symmetry properties under exchange of primed and unprimed variables are now lost. The terms in (5.9) linear in the primed coordinates agree (omitting $\varphi_{\mu}$ ) with the (first-order) results of [20], where the (equivalent) coordinate redefinition $\varphi_{\alpha} \rightarrow \bar{\varphi}_{\alpha} \equiv \varphi_{\alpha}+\eta_{\alpha}$ is given. For the LI one-form associated with the new coordinate we find

$$
\begin{aligned}
\Pi_{\left(\bar{\varphi}_{\alpha}\right)}= & d \bar{\varphi}_{\alpha}-\frac{2}{3}\left(C \Gamma_{\mu}\right)_{\alpha \beta} \theta^{\beta} d x^{\mu}-\frac{2}{3}\left(C \Gamma^{\mu}\right)_{\alpha \beta} \theta^{\beta} d \varphi_{\mu} \\
& +\frac{1}{2}\left(\left(C \Gamma_{\mu}\right)_{\alpha \beta} x^{\mu}+\left(C \Gamma^{\mu}\right)_{\alpha \beta} \varphi_{\mu}+\left(C \Gamma_{\mu}\right)_{\alpha \delta}\left(C \Gamma^{\mu}\right)_{\beta \gamma} \theta^{\gamma} \theta^{\delta}\right) d \theta^{\beta}
\end{aligned}
$$


The manifestly invariant WZ term for the superstring action is given by

$$
S_{W Z}=\int_{W} \phi^{*}(\tilde{b})=\int_{W} \phi^{*}\left(\Pi_{\mu}^{(\varphi)} \Pi^{\mu}+\frac{1}{2} \Pi_{\alpha} \Pi^{\alpha}\right)
$$

which differs from the one in 33 by the term in $\Pi_{\mu}^{(\varphi)} \Pi^{\mu}$. It is immediately checked, using (5.1), (5.5), that $d \tilde{b}=d b=\left(C \Gamma_{\mu}\right)_{\alpha \beta} \Pi^{\mu} \Pi^{\alpha} \Pi^{\beta}$ and hence that $\phi^{*}(\tilde{b})$ and the standard WZ term $\phi^{*}(b)$ are equivalent, differing only by an exact differential. Since the string tension $T$ has dimensions $[T]=M L^{-1}$ and $\left[\Pi_{\mu}^{(\varphi)}\right]=L,\left[\Pi_{\alpha}\right]=L^{3 / 2}$, the products $T \Pi_{\alpha} \Pi^{\alpha}, T \Pi_{\mu}^{(\varphi)} \Pi^{\mu}$, have the dimensions $M L$ of an action.

To compute the conserved Noether currents, we start from (4.21) which gives the closed forms $J^{A}$ on $\tilde{\Sigma}$. With $B^{-1}$ given by the lower right block of $\left(T^{t}\right)^{-1}$ (see (5.7), (4.2)) we get

$$
\begin{aligned}
J^{\mu} & =\left(B^{-1}\right)^{\mu}{ }_{\nu} \Pi^{\nu}+\frac{1}{2}\left(B^{-1}\right)^{\mu}{ }_{\beta} \Pi^{\beta}=\Pi^{\mu}-\frac{1}{2}\left(C \Gamma^{\mu}\right)_{\beta \gamma} \theta^{\gamma} d \theta^{\beta}=d x^{\mu} \\
J^{\alpha} & =\frac{1}{2}\left(B^{-1}\right)^{\alpha}{ }_{\beta} \Pi^{\beta}=\frac{1}{2} d \theta^{\alpha},
\end{aligned}
$$

which, after pulling back on the worldvolume, gives for the charge $Q^{\mu}$ (see (4.23))

$$
Q^{\mu}=\int_{0}^{2 \pi} d \sigma \frac{\partial x^{\mu}}{\partial \sigma}
$$

$Q^{\alpha}$ being zero because we assume that $\theta$ is periodic in $\sigma$. The integral (5.13) may lead to a non-zero result if the topology is nontrivial [12].

\section{2 $D=11$ and the case of the supermembrane}

Our starting point is the FDA of Sec. 3 with $p=2$. We fix the normalisation of the forms by setting $\left(a_{s}, a_{0}, a_{1}, a_{2}\right)=\left(\frac{1}{2}, \frac{1}{2}, 1,-\frac{1}{2}\right)$ so that the dual Lie algebra becomes

$$
\begin{aligned}
\left\{D_{\alpha}, D_{\beta}\right\} & =\left(C \Gamma^{\mu}\right)_{\alpha \beta} X_{\mu}+\left(C \Gamma_{\mu \nu}\right)_{\alpha \beta} Z^{\mu \nu} \\
{\left[X_{\mu}, D_{\alpha}\right] } & =-\left(C \Gamma_{\mu \nu}\right)_{\alpha \beta} Z^{\nu \beta} \\
{\left[X_{\mu}, X_{\nu}\right] } & =\left(C \Gamma_{\mu \nu}\right)_{\alpha \beta} Z^{\alpha \beta} \\
{\left[X_{\mu}, Z^{\lambda \tau}\right] } & =\frac{1}{2} \delta_{\mu}^{[\lambda}\left(C \Gamma^{\tau]}\right)_{\alpha \beta} Z^{\alpha \beta} \\
{\left[D_{\alpha}, Z^{\mu \nu}\right] } & =\left(C \Gamma^{[\mu}\right)_{\alpha \beta} Z^{\nu] \beta} \\
\left\{D_{\alpha}, Z^{\nu \beta}\right\} & =\left(\frac{1}{4}\left(C \Gamma^{\nu}\right)_{\gamma \delta} \delta_{\alpha}^{\beta}+2\left(C \Gamma^{\nu}\right)_{\gamma \alpha} \delta_{\delta}^{\beta}\right) Z^{\gamma \delta},
\end{aligned}
$$

coinciding with that given in 16. The associated extended superspace group manifold is parametrized by the coordinates $\left(\theta^{\alpha}, x^{\mu}, \varphi_{\mu \nu}, \varphi_{\mu \alpha}, \varphi_{\alpha \beta}\right)$ via $^{13}$

$$
g=e^{\theta^{\alpha} D_{\alpha}+x^{\mu} X_{\mu}+\varphi_{\mu \nu} Z^{\mu \nu}+\varphi_{\mu \alpha} Z^{\mu \alpha}+\varphi_{\alpha \beta} Z^{\alpha \beta}},
$$

where $\varphi_{\mu \nu}, \varphi_{\alpha \beta}$ are antisymmetric and symmetric, respectively, in their indices. Application (with the help of FORM) of the BCH formula, where now terms of order five and higher vanish, results in the following group law

$$
\begin{aligned}
\theta^{\alpha^{\prime \prime}}= & \theta^{\alpha^{\prime}}+\theta^{\alpha} \\
x^{\mu \prime \prime}= & x^{\mu^{\prime}}+x^{\mu}-1 / 2 \theta^{\alpha_{2}{ }^{\prime}} \theta^{\alpha_{3}}\left(C \Gamma^{\mu}\right)_{\alpha_{2} \alpha_{3}} \\
\varphi_{\mu_{1} \mu_{2}}^{\prime \prime}= & \varphi_{\mu_{1} \mu_{2}}^{\prime}+\varphi_{\mu_{1} \mu_{2}}-1 / 2 \theta^{\alpha_{3}{ }^{\prime}} \theta^{\alpha_{4}}\left(C \Gamma_{\mu_{1} \mu_{2}}\right)_{\alpha_{3} \alpha_{4}} \\
\varphi_{\mu_{1} \alpha_{2}}^{\prime \prime}= & \varphi_{\mu_{1} \alpha_{2}}^{\prime}-1 / 2 \theta^{\alpha_{3}{ }^{\prime}}\left(C \Gamma^{\mu_{4}}\right)_{\alpha_{3} \alpha_{2}} \varphi_{\mu_{1} \mu_{4}} \\
& -1 / 2 \theta^{\alpha_{3}{ }^{\prime}}\left(C \Gamma_{\mu_{1} \mu_{4}}\right)_{\alpha_{3} \alpha_{2}} x^{\mu_{4}} \\
& +1 / 12 \theta^{\alpha_{3}{ }^{\prime}} \theta^{\alpha_{4}{ }^{\prime}} \theta^{\alpha_{5}}\left(C \Gamma^{\mu_{6}}\right)_{\alpha_{3} \alpha_{2}}\left(C \Gamma_{\mu_{1} \mu_{6}}\right)_{\alpha_{4} \alpha_{5}}
\end{aligned}
$$

\footnotetext{
${ }^{13}$ We use a parametrization different from the one in 16 .
} 


$$
\begin{aligned}
& +1 / 12 \theta^{\alpha_{3}{ }^{\prime}} \theta^{\alpha_{4}{ }^{\prime}} \theta^{\alpha_{5}}\left(C \Gamma^{\mu_{6}}\right)_{\alpha_{4} \alpha_{5}}\left(C \Gamma_{\mu_{1} \mu_{6}}\right)_{\alpha_{3} \alpha_{2}} \pm((1) \leftrightarrow(2)) \\
& \varphi_{\alpha_{1} \alpha_{2}}^{\prime \prime}=\varphi_{\alpha_{1} \alpha_{2}}^{\prime}-1 / 4\left(C \Gamma^{\mu_{3}}\right)_{\alpha_{1} \alpha_{2}} x^{\mu_{4}{ }^{\prime}} \varphi_{\mu_{3} \mu_{4}} \\
& +1 / 2\left(C \Gamma_{\mu_{3} \mu_{4}}\right)_{\alpha_{1} \alpha_{2}} x^{\mu_{3}{ }^{\prime}} x^{\mu_{4}} \\
& +1 / 6 \theta^{\alpha_{3}{ }^{\prime}} \theta^{\alpha_{4}{ }^{\prime}}\left(C \Gamma^{\mu_{5}}\right)_{\alpha_{3} \alpha_{1}}\left(C \Gamma^{\mu_{6}}\right)_{\alpha_{4} \alpha_{2}} \varphi_{\mu_{5} \mu_{6}} \\
& +1 / 6 \theta^{\alpha_{3}{ }^{\prime}} \theta^{\alpha_{4}{ }^{\prime}}\left(C \Gamma^{\mu_{5}}\right)_{\alpha_{3} \alpha_{1}}\left(C \Gamma_{\mu_{5} \mu_{6}}\right)_{\alpha_{4} \alpha_{2}} x^{\mu_{6}} \\
& -1 / 24 \theta^{\alpha_{3}{ }^{\prime}} \theta^{\alpha_{4}{ }^{\prime}} \theta^{\alpha_{5}} \theta^{\alpha_{6}}\left(C \Gamma^{\mu_{7}}\right)_{\alpha_{3} \alpha_{1}}\left(C \Gamma^{\mu_{8}}\right)_{\alpha_{4} \alpha_{6}}\left(C \Gamma_{\mu_{7} \mu_{8}}\right)_{\alpha_{5} \alpha_{2}} \\
& -1 / 24 \theta^{\alpha_{3}{ }^{\prime}} \theta^{\alpha_{4}{ }^{\prime}} \theta^{\alpha_{5}} \theta^{\alpha_{6}}\left(C \Gamma^{\mu_{7}}\right)_{\alpha_{3} \alpha_{1}}\left(C \Gamma^{\mu_{8}}\right)_{\alpha_{5} \alpha_{2}}\left(C \Gamma_{\mu_{7} \mu_{8}}\right)_{\alpha_{4} \alpha_{6}} \\
& -1 / 24 \theta^{\alpha_{3}{ }^{\prime}} \theta^{\alpha_{4}}\left(C \Gamma^{\mu_{5}}\right)_{\alpha_{1} \alpha_{2}}\left(C \Gamma^{\mu_{6}}\right)_{\alpha_{3} \alpha_{4}} \varphi_{\mu_{5} \mu_{6}}^{\prime} \\
& +1 / 48 \theta^{\alpha_{3}{ }^{\prime}} \theta^{\alpha_{4}}\left(C \Gamma^{\mu_{5}}\right)_{\alpha_{1} \alpha_{2}}\left(C \Gamma_{\mu_{5} \mu_{6}}\right)_{\alpha_{3} \alpha_{4}} x^{\mu_{6}{ }^{\prime}} \\
& -1 / 6 \theta^{\alpha_{3}{ }^{\prime}} \theta^{\alpha_{4}}\left(C \Gamma^{\mu_{5}}\right)_{\alpha_{3} \alpha_{1}}\left(C \Gamma^{\mu_{6}}\right)_{\alpha_{4} \alpha_{2}} \varphi_{\mu_{5} \mu_{6}}^{\prime} \\
& -1 / 6 \theta^{\alpha_{3}{ }^{\prime}} \theta^{\alpha_{4}}\left(C \Gamma^{\mu_{5}}\right)_{\alpha_{3} \alpha_{1}}\left(C \Gamma_{\mu_{5} \mu_{6}}\right)_{\alpha_{4} \alpha_{2}} x^{\mu_{6}{ }^{\prime}} \\
& +1 / 48 \theta^{\alpha_{3}{ }^{\prime}} \theta^{\alpha_{4}}\left(C \Gamma^{\mu_{5}}\right)_{\alpha_{3} \alpha_{4}}\left(C \Gamma^{\mu_{6}}\right)_{\alpha_{1} \alpha_{2}} \varphi_{\mu_{5} \mu_{6}}^{\prime} \\
& +1 / 12 \theta^{\alpha_{3}{ }^{\prime}} \theta^{\alpha_{4}}\left(C \Gamma^{\mu_{5}}\right)_{\alpha_{3} \alpha_{4}}\left(C \Gamma_{\mu_{5} \mu_{6}}\right)_{\alpha_{1} \alpha_{2}} x^{\mu_{6}{ }^{\prime}} \\
& -\theta^{\alpha_{3}{ }^{\prime}} \varphi_{\mu_{4} \alpha_{1}}\left(C \Gamma^{\mu_{4}}\right)_{\alpha_{3} \alpha_{2}} \\
& -1 / 8 \theta^{\alpha_{3}{ }^{\prime}} \varphi_{\mu_{4} \alpha_{3}}\left(C \Gamma^{\mu_{4}}\right)_{\alpha_{1} \alpha_{2}} \pm((1) \leftrightarrow(2))
\end{aligned}
$$

(in the expression for $\varphi_{\alpha_{1} \alpha_{2}}$, the r.h.s. is assumed to be symmetrised, with unit weight, in $\alpha_{1}, \alpha_{2}$ ). The $\pm((1) \leftrightarrow(2))$ instruction in the last two expressions means that for each term displayed, one has to add (subtract) a similar term, with the primed and unprimed variables exchanged (taking into account statistics), if the order of the term is odd (even) in the coordinates $\left(e \cdot g \cdot \theta^{\prime \alpha} \varphi_{\mu \beta} \pm((1) \leftrightarrow(2))=\right.$ $\left.\theta^{\prime \alpha} \varphi_{\mu \beta}-\left(-\varphi_{\mu \beta}^{\prime} \theta^{\alpha}\right)\right)$. This symmetry property can be seen to hold in general: from the BCH formula $e^{A^{\prime}} e^{A}=e^{f\left(A^{\prime}, A\right)}$ it follows that $f\left(-A,-A^{\prime}\right)=-f\left(A^{\prime}, A\right)$ and hence, terms of order $n$ in the coordinates are symmetric $(n$ odd) or antisymmetric ( $n$ even) under the above exchange of the two spaces. The linearized (in the primed variables) form of the previous expressions has been given in 20]. Starting from (5.20), we find for the LI vector fields of (5.14)

$$
\begin{aligned}
& D_{\alpha_{1}}=\partial_{\alpha_{1}}+1 / 12 \theta^{\alpha_{2}} \theta^{\alpha_{3}}\left(\left(C \Gamma^{\mu_{4}}\right)_{\alpha_{2} \alpha_{5}}\left(C \Gamma_{\mu_{6} \mu_{4}}\right)_{\alpha_{3} \alpha_{1}}+\left(C \Gamma_{\mu_{4} \mu_{6}}\right)_{\alpha_{2} \alpha_{5}}\left(C \Gamma^{\mu_{4}}\right)_{\alpha_{3} \alpha_{1}}\right) \partial^{\mu_{6} \alpha_{5}} \\
& +1 / 12 \theta^{\alpha_{2}} \varphi_{\mu_{3} \mu_{4}}\left(\left(C \Gamma^{\mu_{3}}\right)_{\alpha_{2} \alpha_{5}}\left(C \Gamma^{\mu_{4}}\right)_{\alpha_{1} \alpha_{6}}+\left(C \Gamma^{\mu_{3}}\right)_{\alpha_{5} \alpha_{6}}\left(C \Gamma^{\mu_{4}}\right)_{\alpha_{2} \alpha_{1}}\right) \partial^{\alpha_{5} \alpha_{6}} \\
& -1 / 3 \theta^{\alpha_{2}} x^{\mu_{3}}\left(C \Gamma^{\mu_{4}}\right)_{\alpha_{2} \alpha_{5}}\left(C \Gamma_{\mu_{3} \mu_{4}}\right)_{\alpha_{6} \alpha_{1}} \partial^{\alpha_{5} \alpha_{6}} \\
& +1 / 8 \theta^{\alpha_{2}} x^{\mu_{3}}\left(C \Gamma^{\mu_{4}}\right)_{\alpha_{5} \alpha_{6}}\left(C \Gamma_{\mu_{3} \mu_{4}}\right)_{\alpha_{2} \alpha_{1}} \partial^{\alpha_{5} \alpha_{6}} \\
& +1 / 2 \theta^{\alpha_{2}}\left(C \Gamma^{\mu_{3}}\right)_{\alpha_{2} \alpha_{1}} \partial_{\mu_{3}} \\
& +\theta^{\alpha_{2}}\left(C \Gamma_{\mu_{3} \mu_{4}}\right)_{\alpha_{2} \alpha_{1}} \partial^{\mu_{3} \mu_{4}} \\
& -1 / 2 x^{\mu_{2}}\left(C \Gamma_{\mu_{2} \mu_{3}}\right)_{\alpha_{1} \alpha_{4}} \partial^{\mu_{3} \alpha_{4}} \\
& -1 / 2 \varphi_{\mu_{2} \mu_{3}}\left(C \Gamma^{\mu_{2}}\right)_{\alpha_{1} \alpha_{4}} \partial^{\mu_{3} \alpha_{4}} \\
& +1 / 8 \varphi_{\mu_{2} \alpha_{1}}\left(C \Gamma^{\mu_{2}}\right)_{\alpha_{3} \alpha_{4}} \partial^{\alpha_{3} \alpha_{4}} \\
& +\varphi_{\mu_{2} \alpha_{3}}\left(C \Gamma^{\mu_{2}}\right)_{\alpha_{1} \alpha_{4}} \partial^{\alpha_{4} \alpha_{3}} \\
& X_{\mu_{1}}=\partial_{\mu_{1}}-1 / 6 \theta^{\alpha_{2}} \theta^{\alpha_{3}}\left(C \Gamma^{\mu_{4}}\right)_{\alpha_{2} \alpha_{5}}\left(C \Gamma_{\mu_{1} \mu_{4}}\right)_{\alpha_{6} \alpha_{3}} \partial^{\alpha_{5} \alpha_{6}} \\
& +1 / 2 \theta^{\alpha_{2}}\left(C \Gamma_{\mu_{1} \mu_{3}}\right)_{\alpha_{2} \alpha_{4}} \partial^{\mu_{3} \alpha_{4}} \\
& +1 / 2 x^{\mu_{2}}\left(C \Gamma_{\mu_{2} \mu_{1}}\right)_{\alpha_{3} \alpha_{4}} \partial^{\alpha_{3} \alpha_{4}} \\
& -1 / 4 \varphi_{\mu_{1} \mu_{2}}\left(C \Gamma^{\mu_{2}}\right)_{\alpha_{3} \alpha_{4}} \partial^{\alpha_{3} \alpha_{4}} \\
& Z^{\mu_{1} \mu_{2}}=\partial^{\mu_{1} \mu_{2}}+1 / 12 \theta^{\alpha_{3}} \theta^{\alpha_{4}}\left(C \Gamma^{\mu_{1}}\right)_{\alpha_{3} \alpha_{5}}\left(C \Gamma^{\mu_{2}}\right)_{\alpha_{4} \alpha_{6}} \partial^{\alpha_{5} \alpha_{6}} \\
& -1 / 12 \theta^{\alpha_{3}} \theta^{\alpha_{4}}\left(C \Gamma^{\mu_{2}}\right)_{\alpha_{3} \alpha_{5}}\left(C \Gamma^{\mu_{1}}\right)_{\alpha_{4} \alpha_{6}} \partial^{\alpha_{5} \alpha_{6}} \\
& +1 / 4 \theta^{\alpha_{3}}\left(C \Gamma^{\mu_{1}}\right)_{\alpha_{3} \alpha_{4}} \partial^{\mu_{2} \alpha_{4}} \\
& -1 / 4 \theta^{\alpha_{3}}\left(C \Gamma^{\mu_{2}}\right)_{\alpha_{3} \alpha_{4}} \partial^{\mu_{1} \alpha_{4}} \\
& +1 / 4 x^{\mu_{1}}\left(C \Gamma^{\mu_{2}}\right)_{\alpha_{3} \alpha_{4}} \partial^{\alpha_{3} \alpha_{4}} \\
& Z^{\mu_{1} \alpha_{2}}=\partial^{\mu_{1} \alpha_{2}}+1 / 8 \theta^{\alpha_{2}}\left(C \Gamma^{\mu_{1}}\right)_{\alpha_{3} \alpha_{4}} \partial^{\alpha_{3} \alpha_{4}}+\theta^{\alpha_{3}}\left(C \Gamma^{\mu_{1}}\right)_{\alpha_{3} \alpha_{4}} \partial^{\alpha_{4} \alpha_{2}}
\end{aligned}
$$




$$
Z^{\alpha_{1} \alpha_{2}}=\partial^{\alpha_{1} \alpha_{2}}
$$

(antisymmetrisation with unit weight in $\mu_{1}, \mu_{2}$ is understood in the r.h.s. of the expression for $Z^{\mu_{1} \mu_{2}}$ ). The manifestly invariant WZ term on the extended superspace $\tilde{\Sigma}$ is given by [16]

$$
\tilde{b}=\frac{2}{3} \Pi_{\mu \nu} \Pi^{\mu} \Pi^{\nu}-\frac{3}{5} \Pi_{\mu \alpha} \Pi^{\mu} \Pi^{\alpha}-\frac{2}{15} \Pi_{\alpha \beta} \Pi^{\alpha} \Pi^{\beta}
$$

$\left([\tilde{b}]=L^{3},[T b]=M L\right)$. It depends on the additional $\varphi$ variables through total differentials since $d \tilde{b}=$ $d b=h=\left(C \Gamma_{\mu \nu}\right)_{\alpha \beta} \Pi^{\mu} \Pi^{\nu} \Pi^{\alpha} \Pi^{\beta}$.

The computation of the full $T$ matrix for the supermembrane is rather tedious. For the Noether currents though (corresponding to the new variables) we only need $B^{-1}$. Reading off the relevant structure constants from (5.14), we find

$$
\begin{aligned}
\rho_{a d j}^{(f)}\left(D_{\alpha}\right) & =\left(\begin{array}{ccc}
0 & 0 & 0 \\
\left(C \Gamma^{\left[\mu_{1}\right.}\right)_{\alpha \alpha_{2}} \delta_{\kappa_{2}}{ }^{\left.\nu_{1}\right]} & 0 & 0 \\
0 & \frac{1}{4}\left(C \Gamma^{\kappa_{1}}\right)_{\beta_{2} \gamma_{2}} \delta_{\alpha}{ }^{\alpha_{1}}+2\left(C \Gamma^{\kappa_{1}}\right)_{\beta_{2}^{\prime} \alpha} \delta_{\gamma_{2}^{\prime}} \alpha_{1} & 0
\end{array}\right), \\
\rho_{\text {adj }}^{(f)}\left(X_{\rho}\right) & =\left(\begin{array}{ccc}
0 & 0 & 0 \\
0 & 0 & 0 \\
\frac{1}{2} \delta_{\rho}^{\left[\mu_{1}\right.}\left(C \Gamma^{\left.\nu_{1}\right]}\right)_{\beta_{2} \gamma_{2}} & 0 & 0
\end{array}\right) .
\end{aligned}
$$

Using these in the exponential in (4.8) we get for $B^{-1}$

$$
B^{-1}=\left(\begin{array}{ccc}
\delta_{\mu_{2} \nu_{2}}^{\mu_{1} \nu_{1}} & -\theta^{\alpha}\left(C \Gamma^{\left[\mu_{1}\right.}\right)_{\alpha \alpha_{2}} \delta^{\left.\nu_{1}\right]} \kappa_{2} & -\frac{1}{2} x^{\left[\mu_{1}\right.}\left(C \Gamma^{\left.\nu_{1}\right]}\right)_{\beta_{2} \gamma_{2}}+\theta^{\alpha} \theta^{\beta}\left(C \Gamma^{\left[\mu_{1}\right.}\right)_{\alpha \gamma_{2}^{\prime}}\left(C \Gamma^{\left.\nu_{1}\right]}\right)_{\beta_{2}^{\prime} \beta} \\
0 & \delta_{\kappa_{2} \alpha_{2}}^{\kappa_{1} \alpha_{1}} & -\frac{1}{4} \theta^{\alpha_{1}}\left(C \Gamma^{\kappa_{1}}\right)_{\beta_{2} \gamma_{2}}-2 \theta^{\alpha}\left(C \Gamma^{\kappa_{1}}\right)_{\beta_{2}^{\prime}{ }^{\prime}} \delta^{\alpha_{1}} \gamma_{2}^{\prime} \\
0 & 0 & \delta_{\beta_{2} \gamma_{2}}^{\beta_{1} \gamma_{1}}
\end{array}\right)
$$

(the external indices are ${ }^{\mu_{1} \nu_{1}} \kappa_{1} \alpha_{1} \beta_{1} \gamma_{1}$ for the rows and $\mu_{2} \nu_{2} \kappa_{2} \alpha_{2} \beta_{2} \gamma_{2}$ for the columns). Substituting now in (4.21) we find for the forms $J^{A}$ on $\Sigma$

$$
\begin{aligned}
J^{\mu_{1} \nu_{1}} & =\frac{2}{3} d x^{\mu_{1}} d x^{\nu_{1}}+\frac{1}{15} \theta^{\alpha}\left(C \Gamma^{\left[\nu_{1}\right.}\right)_{\alpha \beta} d x^{\left.\mu_{1}\right]} d \theta^{\beta}+\frac{1}{15} x^{\left[\mu_{1}\right.}\left(C \Gamma^{\left.\nu_{1}\right]}\right)_{\alpha \beta} d \theta^{\alpha} d \theta^{\beta} \\
& =d\left(\frac{2}{3} x^{\left[\mu_{1}\right.} d x^{\left.\nu_{1}\right]}+\frac{1}{15} \theta^{\alpha} x^{\left[\mu_{1}\right.}\left(C \Gamma^{\left.\nu_{1}\right]}\right)_{\alpha \beta} d \theta^{\beta}\right), \\
J^{\kappa_{1} \alpha_{1}} & =-\frac{3}{5} d x^{\kappa_{1}} d \theta^{\alpha_{1}}-\frac{1}{30}\left(C \Gamma^{\kappa_{1}}\right)_{\alpha_{3} \alpha_{2}} \theta^{\alpha_{3}} d \theta^{\alpha_{2}} d \theta^{\alpha_{1}}+\frac{1}{30}\left(C \Gamma^{\kappa_{1}}\right)_{\alpha_{3} \alpha_{2}} \theta^{\alpha_{1}} d \theta^{\alpha_{3}} d \theta^{\alpha_{2}} \\
& =d\left(\frac{3}{5} d x^{\kappa_{1}} \theta^{\alpha_{1}}-\frac{1}{30}\left(C \Gamma^{\kappa_{1}}\right)_{\alpha_{3} \alpha_{2}} \theta^{\alpha_{3}} \theta^{\alpha_{1}} d \theta^{\alpha_{2}}\right), \\
J^{\beta_{1} \gamma_{1}} & =-\frac{2}{15} d \theta^{\beta_{1}} d \theta^{\gamma_{1}}=d\left(-\frac{2}{15} \theta^{\beta_{1}} d \theta^{\gamma_{1}}\right) .
\end{aligned}
$$

The above locally exact expressions result from rather non-trivial cancellations. The currents are obtained by pulling back to $W$ the forms (5.29). For periodic $\theta$ 's the charges $Q^{\kappa_{1} \alpha_{1}}, Q^{\beta_{1} \gamma_{1}}$ (but not $Q^{\mu_{1} \nu_{1}}$ for a non-trivial two-cycle [12]) turn out to be zero. Thus, this case provides a realization of the algebra (5.14) where only the $Q^{\mu_{1} \nu_{1}}$ term is non-zero.

\section{The case of D-branes}

Let us consider first a bosonic background for which all forms of the $\mathrm{R} \otimes \mathrm{R}$ sector and the dilaton vanish so that the two-form $\mathcal{F} \equiv F-B$, where $F=d A$ and $B$ is the $\mathrm{NS} \otimes \mathrm{NS}$ two-form, reduces to $F$; $A$ is the Born-Infeld one-form on the worldvolume $A(\xi)=A_{i}(\xi) d \xi^{i}$. Then the action of the D $p$-brane reduces to

$$
I=\int d^{p+1} \xi \sqrt{-\operatorname{det}\left(\partial_{i} x^{\mu} \partial_{j} x_{\mu}+F_{i j}\right)}
$$

Let us look for a manifestly supersymmetric generalisation of this action on a suitable extension of flat superspace (we shall consider here only $D=10$, IIA D-branes). For the ordinary $p$-branes the 
supersymmetrisation is achieved by substituting first $\Pi_{i}^{\mu}$ for $\partial_{i} x^{\mu}$ and then by adding a WZ term $b$, $d b=h$, with $h$ characterised [2] by being a non-trivial CE cohomology $(p+2)$-cocycle on superspace $\Sigma$. It was shown in the previous sections how to make these WZ terms manifestly invariant. We shall extend this to the $\mathrm{D} p$-branes case by showing first that the WZ terms may be characterised and classified by CE-cocycles as well, and then by finding manifestly supersymmetric potentials $\tilde{b}$ on the superspaces $\tilde{\Sigma}$ which are obtained by the techniques of Sec. 3 or by dimensional reduction from these. We shall restrict ourselves here to the D2-brane case, and hence to its associated $\tilde{\Sigma}$ parametrised by $\left(\theta^{\alpha}, x^{\mu}, \varphi_{\mu}, \varphi_{\alpha}, \varphi_{\mu \nu}, \varphi_{\mu \alpha}, \varphi_{\alpha \beta}, \varphi\right)$.

\subsection{CE-cocycle classification of D-branes}

The new feature in the $\mathrm{D} p$-brane case is the field $A_{i}(\xi)$ directly defined on the worldvolume. The one-form $A$ transforms under supersymmetry in such a way that $\mathcal{F} \equiv d A-B$ is invariant, where $B$ is a two-form on superspace such that

$$
d B=-\Pi^{\mu}\left(C \Gamma_{\mu} \Gamma_{11}\right)_{\alpha \beta} \Pi^{\alpha} \Pi^{\beta}
$$

Let us now consider $A$ as an abstract form. In our approach, the possible WZ terms will be some non-trivial $(p+2)$-cocycles of the cohomology of a certain FDA (here, of IIA type). This FDA is generated by the supersymmetric invariant $\Pi^{\alpha}, \Pi^{\mu}$ and $\mathcal{F}$ and defined by the structure relations

$$
d \Pi^{\alpha}=0, \quad d \Pi^{\mu}=\frac{1}{2}\left(C \Gamma^{\mu}\right)_{\alpha \beta} \Pi^{\alpha} \Pi^{\beta}, \quad d \mathcal{F}=\Pi^{\mu}\left(C \Gamma_{\mu} \Gamma_{11}\right)_{\alpha \beta} \Pi^{\alpha} \Pi^{\beta} .
$$

Note that $d d \equiv 0$ because the identity $\left(C \Gamma^{\mu} \Gamma_{11}\right)_{\alpha^{\prime} \beta^{\prime}}\left(C \Gamma_{\mu}\right)_{\delta^{\prime} \epsilon^{\prime}}=0$ is valid in $D=10$. The non-trivial $(p+2)$-cocycles are given by closed $(p+2)$-forms $h$ constructed from $\Pi^{\mu}, \Pi^{\alpha}, \mathcal{F}$ that cannot be written as the differential of a $(p+1)$-form constructed from them, and with the same dimensions as the kinetic Lagrangian, i.e. $[h]=L^{p+1}$. This second requirement is necessary to avoid introducing dimensionful constants in the action other than the tension, $[T]=M L^{-p}$.

Since $\mathcal{F}$ is a two-form, $h$ can be expanded in powers of $\mathcal{F}$ as

$$
h=\sum_{n=0}^{\left[\frac{p+2}{2}\right]} \frac{1}{n !} h^{(p+2-2 n)}\left(\Pi^{\mu}, \Pi^{\alpha}\right) \mathcal{F}^{n}
$$

where $h^{(k)}$ is a form of order $k \equiv p+2-2 n$ and, since $D=10, p \leq 8$ excluding the degenerate case $p+1=10$. Moreover, since $\left[h^{(p+2-2 n)}\right]=L^{p+1-2 n}$, the $k$-forms $h^{(k)},\left[h^{(k)}\right]=L^{k-1}$, must be

$$
h^{(k)}=a^{(k)} \Pi^{\mu_{1}} \ldots \Pi^{\mu_{k-2}}\left(C \Gamma_{\mu_{1} \ldots \mu_{k-2}} \Gamma\right)_{\alpha \beta} \Pi^{\alpha} \Pi^{\beta}, \quad k=2, \ldots, p+2,
$$

where $\Gamma=\mathbf{1}$ or $\Gamma_{11}$ so that $\left(C \Gamma_{\mu_{1} \ldots \mu_{k-2}} \Gamma\right)_{\alpha \beta}$ is symmetric $(i . e, k-2=1,2,5,6,9,10$ for $\mathbf{1}$ and $0,1,4,5,8,9$ for $\left.\Gamma_{11}\right)$. Since

$$
d h=\sum_{n=0}^{\left[\frac{p+2}{2}\right]} \frac{1}{n !} d h^{(p+2-2 n)} \mathcal{F}^{n}+\sum_{n=1}^{\left[\frac{p+2}{2}\right]} \frac{1}{(n-1) !}(-1)^{p} h^{(p+2-2 n)}\left(C \Gamma_{\mu} \Gamma_{11}\right)_{\alpha \beta} \Pi^{\mu} \Pi^{\alpha} \Pi^{\beta} \mathcal{F}^{n-1}
$$

the required closure of $h$ is equivalent to the following set of equations

$$
\begin{aligned}
d h^{(p-2[p / 2])} & =0, & & \text { for } n=\left[\frac{p+2}{2}\right] \\
d h^{(p+2-2 n)}+(-1)^{p} h^{(p-2 n)} \Pi^{\mu}\left(C \Gamma_{\mu} \Gamma_{11}\right)_{\alpha \beta} \Pi^{\alpha} \Pi^{\beta} & =0, & & \text { for } n=\left[\frac{p}{2}\right], \ldots, 0 .
\end{aligned}
$$

At this point it is convenient to examine separately the $p$ odd and $p$ even cases.

a) $p$ even

The first eqn. in (6.7) gives $d h^{(0)}=0$. This means $h^{(0)}=0$ because $h^{(0)} \neq 0$ would imply by (6.4) having an additional dimensionful constant, $\left[h^{(0)}\right]=L^{-1}\left([\mathcal{F}]=L^{2}\right)$. For $n=\frac{p}{2}$ the second 
of $(6.7)$ gives $d h^{(2)}=0$. Inserting $h^{(2)}$ from eq. (6.5), we obtain an identity, so $a^{(2)}$ is arbitrary. The remaining equations (for $n=\frac{p-2}{2}$, etc.) are equivalent, by factoring out products of forms $\Pi^{\mu}$ and $\Pi^{\alpha}$, to

$$
\begin{aligned}
& a^{(4)}\left(C \Gamma^{\mu_{2}}\right)_{\alpha^{\prime} \beta^{\prime}}\left(C \Gamma_{\mu_{1} \mu_{2}}\right)_{\delta^{\prime} \epsilon^{\prime}}-a^{(2)}\left(C \Gamma_{11}\right)_{\alpha^{\prime} \beta^{\prime}}\left(C \Gamma_{\mu_{1}} \Gamma_{11}\right)_{\delta^{\prime} \epsilon^{\prime}}=0 \\
& 2 a^{(6)}\left(C \Gamma^{\mu_{4}}\right)_{\alpha^{\prime} \beta^{\prime}}\left(C \Gamma_{\mu_{1} \ldots \mu_{4}} \Gamma_{11}\right)_{\delta^{\prime} \epsilon^{\prime}}-a^{(4)}\left(C \Gamma_{\left[\mu_{1} \mu_{2}\right.}\right)_{\alpha^{\prime} \beta^{\prime}}\left(C \Gamma_{\left.\mu_{3}\right]} \Gamma_{11}\right)_{\delta^{\prime} \epsilon^{\prime}}=0 \\
& 3 a^{(8)}\left(C \Gamma^{\mu_{6}}\right)_{\alpha^{\prime} \beta^{\prime}}\left(C \Gamma_{\mu_{1} \ldots \mu_{6}}\right)_{\delta^{\prime} \epsilon^{\prime}}-a^{(6)}\left(C \Gamma_{\left[\mu_{1} \ldots \mu_{4}\right.} \Gamma_{11}\right)_{\alpha^{\prime} \beta^{\prime}}\left(C \Gamma_{\left.\mu_{5}\right]} \Gamma_{11}\right)_{\delta^{\prime} \epsilon^{\prime}}=0 \\
& 4 a^{(10)}\left(C \Gamma^{\mu_{8}}\right)_{\alpha^{\prime} \beta^{\prime}}\left(C \Gamma_{\mu_{1} \ldots \mu_{8}} \Gamma_{11}\right)_{\delta^{\prime} \epsilon^{\prime}}-a^{(8)}\left(C \Gamma_{\left[\mu_{1} \ldots \mu_{6}\right.}\right)_{\alpha^{\prime} \beta^{\prime}}\left(C \Gamma_{\left.\mu_{7}\right]} \Gamma_{11}\right)_{\delta^{\prime} \epsilon^{\prime}}=0 .
\end{aligned}
$$

Note that the number of identities from $(6.8)$ that are necessary to show that $h$ is closed depends on the values of $p$ since $2 \leq k \leq p+2, k$ even. Specifically, for the D2-brane only the first identity is used, for the D4-brane the first two identities are relevant and the first three (four) identities are required for the existence of the D6-(D8-)branes.

Equations (6.8) have to be identically satisfied for certain values of the $a$ 's to be determined. To find them, one may multiply the equations by $\left(\Gamma^{\nu} C^{-1}\right)^{\beta \delta}$ (although the resulting system is not equivalent to the original one). This procedure gives some equalities between gamma matrices that are only satisfied if $a^{(2)}=-a^{(4)}, a^{(4)}=-6 a^{(6)}, a^{(6)}=-15 a^{(8)}$ and $a^{(8)}=-28 a^{(10)}$. These values are the solution of eqs. (6.8) and determine closed forms $h$ by (6.4), (6.5) provided that the following identities are satisfied

$$
\begin{aligned}
\left(C \Gamma^{\mu_{2}}\right)_{\alpha^{\prime} \beta^{\prime}}\left(C \Gamma_{\mu_{1} \mu_{2}}\right)_{\delta^{\prime} \epsilon^{\prime}}+\left(C \Gamma_{11}\right)_{\alpha^{\prime} \beta^{\prime}}\left(C \Gamma_{\mu_{1}} \Gamma_{11}\right)_{\delta^{\prime} \epsilon^{\prime}}=0 \\
\left(C \Gamma^{\mu_{4}}\right)_{\alpha^{\prime} \beta^{\prime}}\left(C \Gamma_{\mu_{1} \ldots \mu_{4}} \Gamma_{11}\right)_{\delta^{\prime} \epsilon^{\prime}}+3\left(C \Gamma_{\left[\mu_{1} \mu_{2}\right.}\right)_{\alpha^{\prime} \beta^{\prime}}\left(C \Gamma_{\left.\mu_{3}\right]} \Gamma_{11}\right)_{\delta^{\prime} \epsilon^{\prime}}=0 \\
\left(C \Gamma^{\mu_{6}}\right)_{\alpha_{\alpha^{\prime} \beta^{\prime}}}\left(C \Gamma_{\mu_{1} \ldots \mu_{6}}\right)_{\delta^{\prime} \epsilon^{\prime}}+5\left(C \Gamma_{\left[\mu_{1} \ldots \mu_{4}\right.} \Gamma_{11}\right)_{\alpha^{\prime} \beta^{\prime}}\left(C \Gamma_{\left.\mu_{5}\right]} \Gamma_{11}\right)_{\delta^{\prime} \epsilon^{\prime}}=0 \\
\left(C \Gamma^{\mu_{8}}\right)_{\alpha^{\prime} \beta^{\prime}}\left(C \Gamma_{\mu_{1} \ldots \mu_{8}} \Gamma_{11}\right)_{\delta^{\prime} \epsilon^{\prime}}+7\left(C \Gamma_{\left[\mu_{1} \ldots \mu_{6}\right.}\right)_{\alpha^{\prime} \beta^{\prime}}\left(C \Gamma_{\left.\mu_{7}\right]} \Gamma_{11}\right)_{\delta^{\prime} \epsilon^{\prime}}=0,
\end{aligned}
$$

as it is indeed the case (see Appendix B). Therefore we have shown that, for $p$ even, there exist closed $(p+2)$-forms $h$ with the required dimensions for all even values of $p, p \leq 8$.

To prove that the $h$ 's obtained from eq. (6.5) for the appropriate values of $a^{(k)}$ are not CE-trivial, it is sufficient to note that if there were a potential form $b\left(\Pi^{\mu}, \Pi^{\alpha}, \mathcal{F}\right), d b=h$, then this form would be a Lorentz-invariant $(p+1)$-form with physical dimensions $L^{p+1}$, which does not exist for $p+1<10$ since $p$ is even.

b) $p$ odd

In this case, the first eqn. in (6.7) gives $d h^{(1)}=0$. Again, this means $h^{(1)}=0$ because obviously there are no Lorentz-scalar one-forms that can be constructed from $\Pi^{\mu}, \Pi^{\alpha}$ and $\mathcal{F}$. On the other hand, $k$ in $h^{(k)}$ has now the range $3 \leq k \leq p+2, k$ odd. Of these $h^{(k)}$, those corresponding to $k=5$ and $k=9$ vanish independently of the type of the matrix $\Gamma$ (see table). This leaves us with $h^{(3)}$ and $h^{(7)}$ and the second of (6.7) leads to

$$
\begin{aligned}
a^{(3)}\left(C \Gamma^{\mu_{1}}\right)_{\alpha^{\prime} \beta^{\prime}}\left(C \Gamma_{\mu_{1}} \Gamma\right)_{\delta^{\prime} \epsilon^{\prime}} & =0, & a^{(3)}\left(C \Gamma_{\left[\mu_{1}\right.} \Gamma\right)_{\alpha^{\prime} \beta^{\prime}}\left(C \Gamma_{\left.\mu_{2}\right]} \Gamma_{11}\right)_{\delta^{\prime} \epsilon^{\prime}} & =0 \\
a^{(7)}\left(C \Gamma^{\mu_{7}}\right)_{\alpha^{\prime} \beta^{\prime}}\left(C \Gamma_{\mu_{1} \ldots \mu_{5}} \Gamma\right)_{\delta^{\prime} \epsilon^{\prime}} & =0, & a^{(7)}\left(C \Gamma_{\left[\mu_{1} \ldots \mu_{5}\right.} \Gamma\right)_{\alpha^{\prime} \beta^{\prime}}\left(C \Gamma_{\left.\mu_{6}\right]} \Gamma_{11}\right)_{\delta^{\prime} \epsilon^{\prime}} & =0 .
\end{aligned}
$$

In the $a^{(3)}$ equations, $\Gamma$ has to be $\Gamma_{11}$ (the other possibility, $\mathbf{1}_{32}$, may be shown to be inconsistent by multiplying the second expression by $\left.\left(\Gamma^{\nu} C^{-1}\right)^{\beta \delta}\right)$. Multiplying the fourth equation by $\left(\Gamma^{\nu} C^{-1}\right)^{\beta \delta}$ shows that $a^{(7)}=0$ for both $\Gamma=\mathbf{1}, \Gamma_{11}$. Thus we have shown that the only candidate for a WZ term in the odd $p$ case is obtained from $h^{(3)}$ i.e., from

$$
h=\Pi^{\mu}\left(C \Gamma_{\mu} \Gamma_{11}\right)_{\alpha \beta} \Pi^{\alpha} \Pi^{\beta} \mathcal{F}^{\frac{p-1}{2}} \quad, \quad p \geq 1 .
$$

But then $h=d\left(\frac{2}{p+1} \mathcal{F}^{(p+1) / 2}\right)$ by (6.3), and hence is a trivial CE cocycle. Therefore in the $D=10$, IIA theory there are no non-trivial WZ terms for the D-branes with $p$ odd. The other values for $p$ found in our discussion are precisely those for which D-branes of type IIA are known to exist. Thus the IIA D-branes are, as the scalar p-branes [2], characterized by non-trivial CE cocycles.

\footnotetext{
${ }^{14}$ We note in passing that in the heterotic case, for which $N=1\left(\Pi^{\alpha}\right.$ is MW), $a^{(2)}=0$ because $\left(C \Gamma_{11}\right)_{\alpha \beta} \Pi^{\alpha} \Pi^{\beta}=$ $C_{\alpha \beta} \Pi^{\alpha} \Pi^{\beta}=0$ since $\left(\Gamma_{11}\right)^{\alpha}{ }_{\beta} \Pi^{\beta}=\Pi^{\alpha}$. So the chain of equations that follows does not appear and there are no non-trivial WZ terms. This shows, as expected, that there are no D-branes in the heterotic case.
} 


\subsection{D-branes defined on extended superspace}

As we saw, one reason for considering superspace extensions associated with extended objects is that it is possible to find on $\tilde{\Sigma}$ manifestly invariant WZ terms since then $h$ may be expressed as the differential of a LI form $\tilde{b}$. We shall now show that this is also possible for the D2-brane. The starting point is now the FDA given in eq. (2.17) with the generators with more than two vector indices absent, plus the equation for $d \mathcal{F}$ i.e.

$$
\begin{array}{rlrl}
d \Pi^{\alpha} & =0 & d \Pi^{\mu} & =\frac{1}{2}\left(C \Gamma^{\mu}\right)_{\alpha \beta} \Pi^{\alpha} \Pi^{\beta} \\
d \Pi & =\frac{1}{2}\left(C \Gamma_{11}\right)_{\alpha \beta} \Pi^{\alpha} \Pi^{\beta} & d \Pi_{\mu \nu} & =\frac{1}{2}\left(C \Gamma_{\mu \nu}\right)_{\alpha \beta} \Pi^{\alpha} \Pi^{\beta} \\
d \Pi_{\mu}^{(z)} & =\frac{1}{2}\left(C \Gamma_{\mu} \Gamma_{11}\right)_{\alpha \beta} \Pi^{\alpha} \Pi^{\beta} & d \mathcal{F} & =\left(C \Gamma_{\mu} \Gamma_{11}\right)_{\alpha \beta} \Pi^{\mu} \Pi^{\alpha} \Pi^{\beta} .
\end{array}
$$

The reason one should start from $(6.12)$ is that the dual of the algebra defined by the first five equations is the one obtained when one computes the algebra of the Noether charges associated with the supertranslations in the case of the type IIA D2-brane (see [38]).

The next step, as was done in Sec. 3, is extending this algebra with the generators obtained by replacing vector indices by spinorial ones. In the case of the D2-brane this is not difficult to do because, apart from the equation for $d \mathcal{F}$, the free differential algebra one starts with is actually the dimensional reduction of the 11-dimensional one

$$
d \Pi^{\tilde{\mu}}=\frac{1}{2}\left(C \Gamma^{\tilde{\mu}}\right)_{\alpha \beta} \Pi^{\alpha} \Pi^{\beta}, \quad d \Pi_{\tilde{\mu} \tilde{\nu}}=\frac{1}{2}\left(C \Gamma_{\tilde{\mu} \tilde{\nu}}\right)_{\alpha \beta} \Pi^{\alpha} \Pi^{\beta},
$$

in which one sets $\Pi^{\tilde{\mu}} \equiv\left(\Pi^{\mu}, \Pi^{10}=\Pi\right), \Pi_{\tilde{\mu} \tilde{\nu}} \equiv\left(\Pi_{\mu \nu}, \Pi_{\mu 10}=\Pi_{\mu}^{(z)}\right)$. Since this $D=11$ algebra has already been extended recursively in Sec. 3 by the new one-forms $\Pi_{\tilde{\mu} \alpha}$ and $\Pi_{\alpha \beta}$, the extended algebra in $D=10$ is simply its dimensional reduction, for which $\Pi_{\tilde{\mu} \alpha} \equiv\left(\Pi_{\mu \alpha}, \Pi_{\alpha}^{(z)}\right)$. The result is given by eqs. (6.12) plus

$$
\begin{aligned}
d \Pi_{\mu \alpha}= & \left(C \Gamma_{\nu \mu}\right)_{\alpha \beta} \Pi^{\nu} \Pi^{\beta}+\left(C \Gamma_{11} \Gamma_{\mu}\right)_{\alpha \beta} \Pi^{\beta} \Pi^{\beta}+\left(C \Gamma^{\nu}\right)_{\alpha \beta} \Pi_{\nu \mu} \Pi^{\beta}-\left(C \Gamma_{11}\right)_{\alpha \beta} \Pi_{\mu}^{(z)} \Pi^{\beta} \\
d \Pi_{\alpha}^{(z)}= & \left(C \Gamma_{\nu} \Gamma_{11}\right)_{\alpha \beta} \Pi^{\nu} \Pi^{\beta}+\left(C \Gamma^{\nu}\right)_{\alpha \beta} \Pi_{\nu}^{(z)} \Pi^{\beta}, \\
d \Pi_{\alpha \beta}= & -\frac{1}{2}\left(C \Gamma_{\mu \nu}\right)_{\alpha \beta} \Pi^{\mu} \Pi^{\nu}-\left(C \Gamma_{\mu} \Gamma_{11}\right)_{\alpha \beta} \Pi^{\mu} \Pi-\frac{1}{2}\left(C \Gamma^{\mu}\right)_{\alpha \beta} \Pi_{\mu \nu} \Pi^{\nu} \\
& +\frac{1}{2}\left(C \Gamma_{11}\right)_{\alpha \beta} \Pi_{\mu}^{(z)} \Pi^{\mu}-\frac{1}{2}\left(C \Gamma^{\mu}\right)_{\alpha \beta} \Pi_{\mu}^{(z)} \Pi+\frac{1}{4}\left(C \Gamma^{\mu}\right)_{\alpha \beta} \Pi_{\mu \delta} \Pi^{\delta} \\
& +\frac{1}{4}\left(C \Gamma_{11}\right)_{\alpha \beta} \Pi_{\delta}^{(z)} \Pi^{\delta}+2\left(C \Gamma^{\mu}\right)_{\delta \alpha^{\prime}} \Pi_{\mu \beta^{\prime}} \Pi^{\delta}+2\left(C \Gamma_{11}\right)_{\delta \alpha^{\prime}} \Pi_{\beta^{\prime}}^{(z)} \Pi^{\delta}
\end{aligned}
$$

which, apart from the $d \mathcal{F}$ equation, corresponds to the dimensional reduction of (5.14) (with $Z^{\mu}=$ $2 Z^{\mu 10}$ )

$$
\begin{aligned}
\left\{D_{\alpha}, D_{\beta}\right\} & =\left(C \Gamma^{\mu}\right)_{\alpha \beta} X_{\mu}+\left(C \Gamma_{\mu \nu}\right)_{\alpha \beta} Z^{\mu \nu}+\left(C \Gamma_{11}\right)_{\alpha \beta} Z+\left(C \Gamma_{\mu} \Gamma_{11}\right)_{\alpha \beta} Z^{\mu} \\
{\left[X_{\mu}, D_{\alpha}\right] } & =-\left(C \Gamma^{\mu \nu}\right)_{\alpha \beta} Z^{\nu \beta}-\left(C \Gamma_{\mu} \Gamma_{11}\right)_{\alpha \beta} Z^{\beta} \\
{\left[Z, D_{\alpha}\right] } & =\left(C \Gamma_{11} \Gamma_{\mu}\right)_{\alpha \beta} Z^{\beta \mu} \\
{\left[X_{\mu}, X_{\nu}\right] } & =\left(C \Gamma^{\mu \nu}\right)_{\alpha \beta} Z^{\alpha \beta} \\
{\left[Z, X_{\mu}\right] } & =\left(C \Gamma_{11} \Gamma_{\mu}\right)_{\alpha \beta} Z^{\alpha \beta} \\
{\left[X_{\mu}, Z^{\lambda \tau}\right] } & =\frac{1}{2} \delta_{\mu}^{[\lambda}\left(C \Gamma^{\tau]}\right)_{\alpha \beta} Z^{\alpha \beta} \\
{\left[X_{\mu}, Z^{\nu}\right] } & =\frac{1}{2} \delta_{\mu}^{\nu}\left(C \Gamma_{11}\right)_{\alpha \beta} Z^{\alpha \beta} \\
{\left[Z, Z^{\mu}\right] } & =-\frac{1}{2}\left(C \Gamma^{\mu}\right)_{\alpha \beta} Z^{\alpha \beta} \\
{\left[D_{\alpha}, Z^{\mu \nu}\right] } & =\left(C \Gamma^{[\mu}\right)_{\alpha \beta} Z^{\nu] \beta} \\
{\left[D_{\alpha}, Z^{\mu}\right] } & =-\left(C \Gamma_{11}\right)_{\alpha \beta} Z^{\mu \beta}+\left(C \Gamma^{\mu}\right)_{\alpha \beta} Z^{\beta} \\
\left\{D_{\alpha}, Z^{\nu \beta}\right\} & =\left(\frac{1}{4}\left(C \Gamma^{\nu}\right)_{\gamma \delta} \delta_{\alpha}^{\beta}+2\left(C \Gamma^{\nu}\right)_{\gamma \alpha} \delta_{\delta}^{\beta}\right) Z^{\gamma \delta} \\
\left\{D_{\alpha}, Z^{\beta}\right\} & =\left(\frac{1}{1}\left(C \Gamma_{11}\right)_{\gamma \delta} \delta_{\alpha}^{\beta}+2\left(C \Gamma_{11}\right)_{\gamma \alpha} \delta_{\delta}^{\beta}\right) Z^{\gamma \delta} .
\end{aligned}
$$


We can now show, using the new forms in 6.14), that it is possible to find an invariant WZ term $\tilde{b}$, $h=d \tilde{b}$, on the extended superspace. In our case $h$ is given by (eqs. (6.4), (6.5); $k=2,4$ )

$$
h=\left(C \Gamma_{\mu \nu}\right)_{\alpha \beta} \Pi^{\mu} \Pi^{\nu} \Pi^{\alpha} \Pi^{\beta}-\left(C \Gamma_{11}\right)_{\alpha \beta} \Pi^{\alpha} \Pi^{\beta} \mathcal{F} .
$$

Again, it is possible to expand $\tilde{b}$ as $\tilde{b}=b^{(3)}+b^{(1)} \mathcal{F}$. Using this expression in $h=d \tilde{b}$, and identifying the result with $h$, yields $d b^{(1)}=-\left(C \Gamma_{11}\right)_{\alpha \beta} \Pi^{\alpha} \Pi^{\beta}$, from which follows that $b^{(1)}=-2 \Pi$. Similarly,

$$
d b^{(3)}=-2\left(C \Gamma_{\mu} \Gamma_{11}\right)_{\alpha \beta} \Pi \Pi^{\mu} \Pi^{\alpha} \Pi^{\beta}+\left(C \Gamma_{\mu \nu}\right)_{\alpha \beta} \Pi^{\mu} \Pi^{\nu} \Pi^{\alpha} \Pi^{\beta}=\left(C \Gamma_{\tilde{\mu} \tilde{\nu}}\right)_{\alpha \beta} \Pi^{\tilde{\mu}} \Pi^{\tilde{\nu}} \Pi^{\alpha} \Pi^{\beta}
$$

where in the last equality we have rewritten the expression using the eleven-dimensional notation. This has the advantage that the expression for $b^{(3)}$ in $D=11$ was given in $(5.26)$,

$$
b^{(3)}=\frac{2}{3} \Pi_{\tilde{\mu} \tilde{\nu}} \Pi^{\tilde{\mu}} \Pi^{\tilde{\nu}}-\frac{2}{15} \Pi_{\alpha \beta} \Pi^{\alpha} \Pi^{\beta}-\frac{3}{5} \Pi_{\tilde{\mu} \alpha} \Pi^{\tilde{\mu}} \Pi^{\alpha} .
$$

Reducing $(6.18)$ to $D=10$ and adding $b^{(1)} \mathcal{F}=-2 \Pi \mathcal{F}$ we find the invariant WZ term,

$$
\tilde{b}=\frac{2}{3} \Pi_{\mu \nu} \Pi^{\mu} \Pi^{\nu}+\frac{4}{3} \Pi_{\mu}^{(z)} \Pi^{\mu} \Pi-\frac{2}{15} \Pi_{\alpha \beta} \Pi^{\alpha} \Pi^{\beta}-\frac{3}{5} \Pi_{\mu \alpha} \Pi^{\mu} \Pi^{\alpha}-\frac{3}{5} \Pi_{\alpha}^{(z)} \Pi \Pi^{\alpha}-2 \Pi \mathcal{F}
$$

This shows that on the extended superspace corresponding to eqs. (6.12) and (6.14), the WZ term of the type IIA D2-brane can be made invariant, as was the case for the ordinary $p$-branes. We expect that this result holds for the other values of $p$.

In contrast with the case of ordinary $p$-branes, the extended free differential algebra is not the dual of a Lie algebra because of the equation for the two-form $d \mathcal{F}$. However, it is easy to check that

$$
d\left(\frac{1}{2} \Pi^{\alpha} \Pi_{\alpha}^{(z)}-\Pi^{\mu} \Pi_{\mu}^{(z)}\right)=\left(C \Gamma_{\mu} \Gamma_{11}\right)_{\alpha \beta} \Pi^{\mu} \Pi^{\alpha} \Pi^{\beta}
$$

so that, on the extended superspace, we may set

$$
\mathcal{F}=\frac{1}{2} \Pi^{\alpha} \Pi_{\alpha}^{(z)}-\Pi^{\mu} \Pi_{\mu}^{(z)}
$$

in accordance with (6.12), (6.14). This is not a surprising fact since from (6.12) we see that $d \mathcal{F}$ is equal to the $h$ corresponding to the WZ term of the type IIA superstring on a flat background. So it has to be possible to write it as the differential of an invariant form $\tilde{b}=\tilde{b}\left(\Pi^{\mu}, \Pi^{\alpha}, \Pi_{\mu}^{(z)}, \Pi_{\alpha}^{(z)}\right)$ on the fully extended superspace $\tilde{\Sigma}$ of the IIA superstring. Since $\mathcal{F}=d A-B$ and $B$ is defined on $\Sigma, d A$ may be written on $\tilde{\Sigma}$. Making use of its LI forms i.e., of

$$
\begin{array}{rlrl}
\Pi^{\alpha} & =d \theta^{\alpha}, & \Pi^{\mu}= & d x^{\mu}+\frac{1}{2}\left(C \Gamma^{\mu}\right)_{\alpha \beta} \theta^{\alpha} d \theta^{\beta} \\
\Pi_{\mu}^{(z)}=d \varphi_{\mu}+\frac{1}{2}\left(C \Gamma_{\mu} \Gamma_{11}\right)_{\alpha \beta} \theta^{\alpha} d \theta^{\beta}, & \Pi_{\alpha}^{(z)}= & d \varphi_{\alpha}-\left(C \Gamma_{\mu} \Gamma_{11}\right)_{\alpha \beta} d x^{\mu} \theta^{\beta}-\left(C \Gamma^{\mu}\right)_{\alpha \beta} d \varphi_{\mu} \theta^{\beta} \\
& -\frac{1}{6}\left[\left(C \Gamma_{\mu} \Gamma_{11}\right)_{\alpha \beta}\left(C \Gamma^{\mu}\right)_{\delta \epsilon}+\left(C \Gamma_{\mu} \Gamma_{11}\right)_{\delta \epsilon}\left(C \Gamma^{\mu}\right)_{\alpha \beta}\right] \theta^{\beta} \theta^{\delta} d \theta^{\epsilon},
\end{array}
$$

it is easy to identify $A$ as the one-form on $\tilde{\Sigma}$

$$
A=\varphi_{\mu} d x^{\mu}+\frac{1}{2} \varphi_{\alpha} d \theta^{\alpha}
$$

In this way, the customary Born-Infeld worldvolume field $A_{i}(\xi)$ becomes here $\phi^{*}(A)$, with $A$ on $\tilde{\Sigma}$ given by (6.23), and its existence may be looked at as a consequence of extended supersymmetry.

The previous discussion shows that it is natural to rewrite the action of the D-branes on a flat background by using only objects that are initially defined on the appropriately extended superspace. We show now that the Euler Lagrange (EL) equations are still the same (provided a rather natural condition is met) and that the gauge transformations of $A_{i}(\xi)$ can be reinterpreted in the new language. So at this point it seems that the geometric difference between the ordinary $p$-branes and the D $p$-branes is that while the action of the former may be defined from forms on ordinary superspace $\Sigma$, the action 
of the latter requires the extended superspace of the IIA superstring if one whishes to avoid objects that only have a meaning on the worldvolume. In the IIA superstring case the extended superspace was also considered, but the new variables appeared only in the WZ term and as total derivatives (Sec. 5.1) and thus had trivial EL equations. In the D-brane case, in contrast, these variables have nontrivial EL equations.

Let us now see how the EL equations change by making the substitution $A \rightarrow \varphi_{\mu} d x^{\mu}+\frac{1}{2} \varphi_{\alpha} d \theta^{\alpha}$. Let $I\left[x^{\mu}, \theta^{\alpha}, A_{i}\right]$ be the action before making the subtitution, where $A_{i}$ are the coordinates of the form $A\left(A=A_{i} d \xi^{i}\right)$. The EL equations are

$$
\frac{\delta I}{\delta x^{\mu}}=0, \quad \frac{\delta I}{\delta \theta^{\alpha}}=0, \quad \frac{\delta I}{\delta A_{j}}=0 .
$$

When the substitution is made, there are new terms in the equations, and they read

$$
\begin{aligned}
\int d \xi^{\prime p+1} \frac{\delta I}{\delta A_{j}\left(\xi^{\prime}\right)} \frac{\delta A_{j}\left(\xi^{\prime}\right)}{\delta x^{\mu}(\xi)}+\frac{\delta I}{\delta x^{\mu}} & =0, & \frac{\delta I}{\delta \varphi_{\mu}}=\frac{\delta I}{\delta A_{j}} \partial_{j} x^{\mu} & =0 \\
\int d \xi^{\prime p+1} \frac{\delta I}{\delta A_{j}\left(\xi^{\prime}\right)} \frac{\delta A_{j}\left(\xi^{\prime}\right)}{\delta \theta^{\alpha}(\xi)}+\frac{\delta I}{\delta \theta^{\alpha}} & =0, & \frac{\delta I}{\delta \varphi_{\alpha}}=\frac{1}{2} \frac{\delta I}{\delta A_{j}} \partial_{j} \theta^{\alpha} & =0
\end{aligned}
$$

where the additional contributions come from the partial functional derivative terms. If $\frac{\delta I}{\delta A_{j}} \partial_{j} x^{\mu}$ and $\frac{\delta I}{\delta A_{j}} \partial_{j} \theta^{\alpha}$ were zero without $\frac{\delta I}{\delta A_{j}}$ being zero, this would imply the collapse of one worldvolume dimension. Thus, we must have $\frac{\delta I}{\delta A_{j}}=0$ which in the first equation of each set in 6.25) implies eqs. 6.24. Hence both actions are equivalent. In fact, it may be shown that there is an additional gauge freedom which accounts for the difference of degrees of freedom between $A$ (eqn. (6.23)) and $A_{i}(\xi)$, but we shall not discuss this here ${ }^{\text {T. }}$. This seems to indicate that, when the action on $W$ is obtained from entities on $\tilde{\Sigma}$, there is an additional gauge freedom which in our formulation plays a rôle complementing that of $\kappa$-symmetry.

Finally, the $U(1)$ gauge field $A_{i}(\xi)$ on $W$ has a gauge transformation $\delta A_{i}(\xi)=\partial_{i} \Lambda(\xi)$. The question now is what is the gauge transformation of the component fields if one writes $A_{i} d \xi^{i}$ as $\phi^{*}(A)$. In other words, for a given $\Lambda(\xi)$, there should be a transformation of $\varphi_{\mu}$ and $\varphi_{\alpha}$ in (6.23) reproducing $\delta_{i} \Lambda$. This may be obtained by means of a superfield $\lambda$ such that $\phi^{*} \lambda\left(x^{\mu}, \theta^{\alpha}\right)=\Lambda(\xi)$. Then if under a gauge transformation one defines $\delta \varphi_{\mu}=\partial_{\mu} \lambda$ and $\delta \varphi_{\alpha}=2 \partial_{\alpha} \lambda, \phi^{*}(A)$ behaves as expected since then $\delta \phi^{*}\left[\varphi_{\mu} d x^{\mu}+\frac{1}{2} \varphi_{\alpha} d \theta^{\alpha}\right]=\partial_{i} \Lambda$. The fact that when the supersymmetry transformations of a field close only modulus a gauge transformation one obtains an extension of a FDA is not restricted to $\mathrm{D} p$-branes. In fact, one may achieve manifest invariance by introducing an electromagnetic potential on the worldsheet in the Green-Schwarz superstring action, in which case the string tension is the circulation of the potential around the string [49] (see also [50]) and a similar result applies to the other scalar $p$-branes [51]. Clearly, the worldvolume fields introduced there could be defined on our appropriate extended superspaces as well. As for the IIB D $p$-brane, an analysis similar to that in this section for the IIA case would classify them by first showing that WZ terms exist for odd $p$. In a second stage, the worldvolume gauge field $A$ may be expressed as the pull-back of a IIB superspace one-form. In fact, this last point for the $A$ in the $p=1$ IIB D-string case has been discussed very recently in [22] by introducing an appropriate extended group manifold. We may conclude, then, that the different worldvolume fields may be expressed in terms of forms defined on suitably extended superspaces.

\section{Noether charges and D-brane actions}

It follows from the discussion of Sec. 6 that the worldvolume field $A(\xi)$ that appears in the D2-brane action may be written on the superstring extended superspace parametrized by $\left(x^{\mu}, \theta^{\alpha}, \varphi_{\mu}, \varphi_{\alpha}\right)$. On the other hand, the D2-WZ term, which is quasi-invariant in these coordinates, can be made strictly invariant by further extending the previous superspace to $\tilde{\Sigma}=\left(\theta^{\alpha}, x^{\mu}, \varphi_{\mu}, \varphi_{\alpha}, \varphi_{\mu \nu}, \varphi_{\mu \alpha}, \varphi_{\alpha \beta}, \varphi\right)$. In this way, the whole action is invariant.

\footnotetext{
${ }^{15}$ We thank P. Townsend for discussions on this point.
} 
If one now computes the canonical commutators (or Poisson brackets) of the charges corresponding to the symmetries of the action, the resulting algebra is exactly the RI version of the Lie algebra dual to (6.12) (removing its last line) plus (6.14), given in (6.15). The RI generator algebra $(\{Q, Q\}$, etc.) is the same as (6.15) with an additional minus sign on the r.h.s. Let us concentrate on the $\left\{Q_{\alpha}, Q_{\beta}\right\}$ commutator,

$$
\left\{Q_{\alpha}, Q_{\beta}\right\}=\left(C \Gamma^{\mu}\right)_{\alpha \beta} P_{\mu}+\left(C \Gamma_{\mu} \Gamma_{11}\right)_{\alpha \beta} \hat{Z}^{\mu}+\left(C \Gamma_{\mu \nu}\right)_{\alpha \beta} \hat{Z}^{\mu \nu}+\left(C \Gamma_{11}\right)_{\alpha \beta} \hat{Z}
$$

(there has been a redefinition of the generators so that $\{Q, Q\}=+C \Gamma^{\mu} P_{\mu}$ etc). Let us assume that we had written the action, as it is customary, in terms of $\left(x^{\mu}, \theta^{\alpha}, A\right)$ alone, with $A=A(\xi)$ directly defined on $W$. In this case, the $C \Gamma_{\mu \nu}$ and $C \Gamma_{11}$ contributions would come from the quasi-invariance of the WZ Lagrangian, while $C \Gamma_{11} \Gamma_{\mu}$ would be the result of the contribution of the $A(\xi)$ field to the Noether current [38] (see also [48]). This follows easily from the appropriate definition of the conserved Noether currents and charges (see, e.g., [26]) which include an additional piece when the Lagrangian is quasi-invariant, a common feature of the conventional actions for $p$-branes [12]. In the present D-branes case, there is an additional contribution due to the worldvolume field $A(\xi)$ since its transformation properties, $\delta A=\Delta$, are postulated to compensate for those of the composite object $B$, $\delta B=d \Delta$, so that $\mathcal{F}=d A-B$ is invariant. As a result, the supersymmetry transformations do not close on $A$, and this produces an additional term by a mechanism similar to the one in the standard quasi-invariance case.

These modifications become evident in our context i.e., by formulating the action on the extended superspace. Let us consider the D2-brane Lagrangian with the quasi-invariant WZ term $b=b\left(x^{\mu}, \theta^{\alpha}, \varphi_{\mu}, \varphi_{\alpha}\right)$. The conserved Noether currents then have to include the quasi-invariance piece. If we wrongly ignored this additional term, the (canonical formalism) algebra of the corresponding (non-conserved, non-Noether) charges would be the algebra of the symmetries $x^{\mu}, \theta^{\alpha}, \varphi_{\mu}, \varphi_{\alpha}$ of the Lagrangian, i.e.

$$
\left\{Q_{\alpha}, Q_{\beta}\right\}=\left(C \Gamma^{\mu}\right)_{\alpha \beta} P_{\mu}+\left(C \Gamma_{\mu} \Gamma_{11}\right)_{\alpha \beta} \hat{Z}^{\mu}
$$

The algebra of the conserved Noether charges is not (7.2), however, because these must include the quasi-invariance contribution. We may find the correct algebra immediatley by replacing the quasiinvariant WZ term $b$, by $\tilde{b}=\tilde{b}\left(x^{\mu}, \theta^{\alpha}, \varphi_{\mu}, \varphi_{\alpha}, \varphi_{\mu \nu}, \varphi_{\mu \alpha}, \varphi_{\alpha \beta}, \varphi\right)$, which is manifestly invariant since the transformation properties of the additional variables $\left(\varphi_{\mu \nu}, \varphi_{\mu \alpha}, \varphi_{\alpha \beta}, \varphi\right)$ remove the quasi-invariance of $b$. By definition, the transformation properties of all the coordinates obviously close into the group law or algebra. Hence, it follows that the algebra of charges computed using the canonical formalism reproduces (7.1), and that the contributions to $\hat{Z}^{\mu \nu}$ and $\hat{Z}$ are entirely due to the WZ term $\tilde{b}$ (or to the quasi-invariance of $b\left(x^{\mu}, \theta^{\alpha}, \varphi_{\mu}, \varphi_{\alpha}\right)$ if we used $b$ instead).

\section{Branes with higher order tensors: the case of the M5-brane}

We shall now show that the previous analysis can be applied also to other extended objects that are neither ordinary $p$-branes nor D-branes. We shall consider here the case of the $D=11 M 5$-brane, which contains a worldvolume two-form field $A$ in the action (see [52]). The action in a flat bosonic background depends on $A$ trough $H=d A-C$, where $C$ is a background three-form. We shall take as our starting point the case with $C=0$ and with all other forms of rank higher than one in that action vanishing. We do not need to worry about the (generalized) self-duality condition for A on the worldvolume, since this condition may arise as a field equation for an auxiliary field (see 52, 53 ). The supersymmetric action of the M5-brane is obtained in two steps. First, one substitutes $H=d A-C$ for $d A$, where $C$ is a form on ordinary flat superspace such that

$$
d C=-\left(C \Gamma_{\mu \nu}\right)_{\alpha \beta} \Pi^{\mu} \Pi^{\nu} \Pi^{\alpha} \Pi^{\beta},
$$

and the transformation properties of the worldvolume field $A$ are fixed so that $H$ is invariant. Secondly, a WZ term is added to obtain $\kappa$ symmetry. 
Let us now find the WZ term in our framework. It should be obtained from the FDA generated by the abstract invariant forms $\Pi^{\alpha}, \Pi^{\mu}, H$,

$$
d \Pi^{\alpha}=0, \quad d \Pi^{\mu}=\frac{1}{2}\left(C \Gamma^{\mu}\right)_{\alpha \beta} \Pi^{\alpha} \Pi^{\beta}, \quad d H=\left(C \Gamma_{\mu \nu}\right)_{\alpha \beta} \Pi^{\mu} \Pi^{\nu} \Pi^{\alpha} \Pi^{\beta},
$$

and be given by a CE-non-trivial potential $b$ of a closed form $h\left(\Pi^{\alpha}, \Pi^{\mu}, H\right)$. Thus, we have to solve the problem of finding nontrivial $(p+2)$-cocycles of the FDA (8.2). We shall find that there is no solution unless $p=5$.

A general $(p+2)$-form on (8.2) can be written as

$$
h=h^{(p+2)}+h^{(p-1)} H \quad ;
$$

there are no further powers of $H$ since $H^{2} \equiv H \wedge H=0$. The closure of $h$ gives

$$
d h^{(p+2)}+d h^{(p-1)} H-(-1)^{p} h^{(p-1)}\left(C \Gamma_{\mu \nu}\right)_{\alpha \beta} \Pi^{\mu} \Pi^{\nu} \Pi^{\alpha} \Pi^{\beta}=0,
$$

which is equivalent to

$$
\begin{aligned}
& d h^{(p-1)}=0 \\
& d h^{(p+2)}=(-1)^{p} h^{(p-1)}\left(C \Gamma_{\mu \nu}\right)_{\alpha \beta} \Pi^{\mu} \Pi^{\nu} \Pi^{\alpha} \Pi^{\beta} .
\end{aligned}
$$

Now, since $[h]=L^{p+1}$ and $[H]=L^{3}$,

$$
\begin{aligned}
& h^{(p+2)}=a^{(p+2)}\left(C \Gamma_{\mu_{1} \ldots \mu_{p}}\right)_{\alpha \beta} \Pi^{\mu_{1}} \ldots \Pi^{\mu_{p}} \Pi^{\alpha} \Pi^{\beta} \\
& h^{(p-1)}=a^{(p-1)}\left(C \Gamma_{\mu_{1} \ldots \mu_{p-3}}\right)_{\alpha \beta} \Pi^{\mu_{1}} \ldots \Pi^{\mu_{p-3}} \Pi^{\alpha} \Pi^{\beta},
\end{aligned}
$$

for some constants $a^{(p+2)}$ and $a^{(p-1)}$. The first equation in $(8.5)$ requires $a^{(p-1)}=0$ unless $p-3=2$, in which case $a^{(p-1)}$ is arbitrary due to the identity $\left(C \Gamma_{\mu \nu}\right)_{\alpha^{\prime} \beta^{\prime}}\left(C \Gamma^{\nu}\right)_{\delta^{\prime} \epsilon^{\prime}}=0$, valid in $D=4,5,7,11$. If $p \neq 5, a^{(p-1)}=0$ and $h^{(p-1)}=0$, and the second equation of (8.5) gives $d h^{(p+2)}=0$, which again implies $a^{(p+2)}=0$ unless $p=2$. But if $p=2$, we have $h=h^{(2+2)} \propto\left(C \Gamma_{\mu \nu}\right)_{\alpha \beta} \Pi^{\mu} \Pi^{\nu} \Pi^{\alpha} \Pi^{\beta}=d H$, in which case $h$ is the differential of a LI form and hence CE-trivial. Thus we are just left with the case $p=5, a^{(5-1)}$ arbitrary. Inserting (8.6) in 8.5 gives, factoring out the $\Pi^{\alpha}$ 's and $\Pi^{\mu}$ 's,

$$
\frac{5}{2} a^{(5+2)}\left(C \Gamma^{\mu_{5}}\right)_{\alpha^{\prime} \beta^{\prime}}\left(C \Gamma_{\mu_{1} \ldots \mu_{5}}\right)_{\delta^{\prime} \epsilon^{\prime}}+a^{(5-1)}\left(C \Gamma_{\left[\mu_{1} \mu_{2}\right.}\right)_{\alpha^{\prime} \beta^{\prime}}\left(C \Gamma_{\left.\mu_{3} \mu_{4}\right]}\right)_{\delta^{\prime} \epsilon^{\prime}}=0 \quad \text {. }
$$

The second identity in (B.1) gives $a^{(7)}=-\frac{2}{15} a^{(4)}$. The resulting closed form

$$
h \propto\left(C \Gamma_{\mu_{1} \ldots \mu_{5}}\right)_{\alpha \beta} \Pi^{\mu_{1}} \ldots \Pi^{\mu_{5}} \Pi^{\alpha} \Pi^{\beta}-\frac{15}{2}\left(C \Gamma_{\mu_{1} \mu_{2}}\right)_{\alpha \beta} \Pi^{\mu_{1}} \Pi^{\mu_{2}} \Pi^{\alpha} \Pi^{\beta} H
$$

is not CE-exact, as may be seen by an argument analogous to that used in the IIA D-branes case: a LI potential form $b$ would have to be a scalar six-form depending on $\Pi^{\alpha}, \Pi^{\mu}$ and $H$ with dimensions $L^{6}$, which does not exist.

It is possible to see, however, that a LI expression for $H$ exists on the appropriate extended superspace. Since $H$ is a three-form, it has formally the same properties as the invariant WZ term $\tilde{b}$ of the M2-brane, the extended superspace of which is the one corresponding to the Lie FDA obtained by the methods of Sec. 3 . Eqs. (2.3), (3.1), (3.4) and the first of (3.5) with $\left(a_{s}, a_{0}, a_{1}, a_{2}\right)=\left(\frac{1}{2}, \frac{1}{2}, 1,-\frac{1}{2}\right)$ give, respectively,

$$
\begin{aligned}
& d \Pi^{\alpha}=0, \\
& d \Pi_{\mu \nu}=\frac{1}{2}\left(C \Gamma_{\mu \nu}\right)_{\alpha \beta} \Pi^{\alpha} \Pi^{\beta}, \\
& d \Pi^{\mu}=\frac{1}{2}\left(C \Gamma^{\mu}\right)_{\alpha \beta} \Pi^{\alpha} \Pi^{\beta} \\
& d \Pi_{\mu \alpha}=\left(C \Gamma_{\nu \mu}\right)_{\alpha \beta} \Pi^{\nu} \Pi^{\beta}+\left(C \Gamma^{\nu}\right)_{\alpha \beta} \Pi_{\nu \mu} \Pi^{\beta}, \\
& d \Pi_{\alpha \beta}=-\frac{1}{2}\left(C \Gamma_{\mu \nu}\right)_{\alpha \beta} \Pi^{\mu} \Pi^{\nu}-\frac{1}{2}\left(C \Gamma^{\mu}\right)_{\alpha \beta} \Pi_{\mu \nu} \Pi^{\nu} \\
& +\frac{1}{4}\left(C \Gamma^{\mu}\right)_{\alpha \beta} \Pi_{\mu \delta} \Pi^{\delta}+2\left(C \Gamma^{\mu}\right)_{\delta \alpha^{\prime}} \Pi_{\mu \beta^{\prime}} \Pi^{\delta},
\end{aligned}
$$

i.e., the dual of (5.14)). Using again (5.26) we may then write

$$
H=\frac{2}{2} \Pi^{\mu} \Pi^{\nu} \Pi_{\mu \nu}+\frac{3}{5} \Pi^{\mu} \Pi^{\alpha} \Pi_{\mu \alpha}-\frac{2}{15} \Pi_{\alpha \beta} \Pi^{\alpha} \Pi^{\beta} .
$$


We might now go on and show that there exists a LI $\tilde{b}$ such that $h=d \tilde{b}$ on a suitably extended superspace; we shall omit its expression [19] since it is not needed below. What we wish to show is that now we may use (8.10) to replace the worldvolume two-form $A(\xi)$ by the pull-back of the two-form $A$ on extended superspace given by

$$
\begin{aligned}
A= & \frac{2}{3} \varphi_{\mu \nu} d x^{\mu} d x^{\nu}-\frac{3}{5} \varphi_{\mu \alpha} d x^{\mu} d \theta^{\alpha}-\frac{2}{15} \varphi_{\alpha \beta} d \theta^{\alpha} d \theta^{\beta} \\
& +\frac{1}{30} \varphi_{\mu \nu} x^{\mu}\left(C \Gamma^{\nu}\right)_{\alpha \beta} d \theta^{\alpha} d \theta^{\beta}+\frac{11}{30} \varphi_{\mu \nu} d x^{\mu}\left(C \Gamma^{\nu}\right)_{\alpha \beta} \theta^{\alpha} d \theta^{\beta}-\frac{13}{180} \varphi_{\mu \nu}\left(C \Gamma^{\mu}\right)_{\alpha \beta}\left(C \Gamma^{\nu}\right)_{\delta \epsilon} \theta^{\alpha} d \theta^{\beta} \theta^{\delta} d \theta^{\epsilon} \\
& +\frac{1}{10} \varphi_{\mu \alpha}\left(C \Gamma^{\mu}\right)_{\delta \epsilon} \theta^{\delta} d \theta^{\epsilon} d \theta^{\alpha}+\frac{1}{20} \varphi_{\mu \alpha}\left(C \Gamma^{\mu}\right)_{\delta \epsilon} d \theta^{\delta} d \theta^{\epsilon} \theta^{\alpha}
\end{aligned}
$$

Again, this expression may also be used to find the gauge transformation $\delta A(\xi)=d \Lambda(\xi)$. This is achieved by the one-form on superspace $\lambda=\lambda_{\mu} d x^{\mu}+\lambda_{\alpha} d \theta^{\alpha}, \phi^{*}(\lambda)=\Lambda(\xi)$. Then, if

$$
\begin{aligned}
\delta \varphi_{\mu \nu}= & \frac{3}{2} \partial_{[\mu} \lambda_{\nu]}, \\
\delta \varphi_{\mu \alpha}= & -\frac{5}{3}\left(\partial_{\mu} \lambda_{\alpha}+\partial_{\alpha} \lambda_{\mu}\right)+\frac{11}{12} \partial_{[\mu} \lambda_{\nu]}\left(C \Gamma^{\nu}\right)_{\alpha \beta} \theta^{\beta}, \\
\delta \varphi_{\alpha \beta}= & -\frac{15}{2} \partial_{\alpha^{\prime}} \lambda_{\beta^{\prime}}+\frac{15}{21}\left(C \Gamma^{\mu}\right)_{\alpha \beta} \theta^{\delta}\left(\partial_{\mu} \lambda_{\delta}+\partial_{\delta} \lambda_{\mu}\right)+\frac{15}{12}\left(C \Gamma^{\mu}\right)_{\delta \alpha^{\prime}} \theta^{\delta}\left(\partial_{\mu} \lambda_{\beta^{\prime}}+\partial_{\beta^{\prime}} \lambda_{\mu}\right) \\
& -\frac{139}{240}\left(C \Gamma^{\mu}\right)_{\delta \beta}\left(C \Gamma^{\nu}\right)_{\alpha \epsilon} \theta^{\delta} \theta^{\epsilon} \partial_{[\mu} \lambda_{\nu]}+\frac{1}{20} x^{\mu}\left(C \Gamma^{\nu}\right)_{\alpha \beta} \partial_{[\mu} \lambda_{\nu]}
\end{aligned}
$$

one obtains $\delta \phi^{*}(A)=d \Lambda(\xi)$.

As in the previous D-brane case, the EL equations derived from the action $I\left[x^{\mu}, \theta^{\alpha}, A_{i j}\right]$,

$$
\frac{\delta I}{\delta x^{\mu}}=0 \quad, \quad \frac{\delta I}{\delta \theta^{\alpha}}=0 \quad, \quad \frac{\delta I}{\delta A_{i j}}=0,
$$

are equivalent to the ones corresponding to the new action in which $A(\xi)$ is the pull-back of (8.11). Indeed, the equation for $\varphi_{\alpha \beta}$ gives $\frac{\delta I}{\delta A_{i j}} \partial_{i} \theta^{\alpha} \partial_{j} \theta^{\beta}=0$, and substituting it into that of $\varphi_{\mu \alpha}$,

$$
\frac{\delta I}{\delta A_{i j}}\left(-\frac{3}{5} \partial_{i} x^{\mu} \partial_{j} \theta^{\alpha}+\frac{1}{10}\left(C \Gamma^{\mu}\right)_{\delta \epsilon} \theta^{\delta} \partial_{i} \theta^{\epsilon} \partial_{j} \theta^{\alpha}+\frac{1}{20}\left(C \Gamma^{\mu}\right)_{\delta \epsilon} \theta^{\alpha} \partial_{i} \theta^{\delta} \partial_{j} \theta^{\epsilon}\right)=0
$$

gives $\frac{\delta I}{\delta A_{i j}} \partial_{i} x^{\mu} \partial_{j} \theta^{\alpha}=0$ and so on. Therefore one obtains

$$
\begin{aligned}
& \int d \xi^{\prime p+1} \frac{1}{2} \frac{\delta I}{\delta A_{i j}\left(\xi^{\prime}\right)} \frac{\delta A_{i j}\left(\xi^{\prime}\right)}{\delta x^{\mu}(\xi)}+\frac{\delta I}{\delta x^{\mu}}=0, \quad \frac{\delta I}{\delta A_{i j}} \partial_{i} x^{\mu} \partial_{j} x^{\nu}=0, \\
& \int d \xi^{\prime p+1} \frac{1}{2} \frac{\delta I}{\delta A_{i j}\left(\xi^{\prime}\right)} \frac{\delta A_{i j}\left(\xi^{\prime}\right)}{\delta \theta^{\alpha}(\xi)}+\frac{\delta I}{\delta \theta^{\alpha}}=0, \quad \frac{\delta I}{\delta A_{i j}} \partial_{i} x^{\mu} \partial_{j} \theta^{\alpha}=0, \quad \frac{\delta I}{\delta A_{i j}} \partial_{i} \theta^{\alpha} \partial_{j} \theta^{\beta}=0 .
\end{aligned}
$$

The second equation implies $\frac{\delta I}{\delta A_{i j}} \partial_{i} x^{\mu}=0$ for all $\mu$ if one wants to avoid the possibility of one dimension of the object collapsing. This in turn implies $\frac{\delta I}{\delta A_{i j}}=0$ for the same reason, and inserting this equation into (8) gives (8.13).

\section{Conclusions}

We have provided in this paper a unified approach to the study of various $p$-branes by defining them on suitably extended superspaces. All of these are supergroup manifolds, extensions of the basic odd abelian groups $\mathrm{s} \operatorname{Tr}_{D}$ determined by the spinors of the specific theory considered. The extension algorithms in Secs. 2.2 and 3 show how they depend, when they do, on specific identities for $\Gamma$ matrices. The central extensions do not need any $\Gamma$-identities, but the non-central ones require the identities (3.3), precisely the ones needed to define the WZ terms of the old branescan.

The centrally extended superspaces are associated with (topological) charges, but the introduction of manifestly supersymmetric WZ terms requires the addition of non-central variables, already for the 
branes of the old branescan. When the procedure is applied to D $p$-branes, it is seen that all the fields in their action may also be defined by pullbacks of entities on the previously introduced superspaces. In the language of FDA's, our results show that all the FDA's involved in the formulation of the p-branes considered here become Lie FDA's on suitably extended superspaces. We conjecture, in view of the previous discussion, that this is the case in general and that there exists an extended superspace definition for all fields appearing in the action of the various $p$-branes. In other words, there exists a kind of field/extended superspaces democracy by which all brane worldvolume fields are pullbacks from some target superspace $\tilde{\Sigma}$. The appropriate $\tilde{\Sigma}$ of the theory is given by an extension of a certain $\operatorname{sTr}_{D}$ and, using $\tilde{\Sigma}$, the action can be defined in a manifestly invariant form. In fact, in this field/extended superspace democracy context, the invariance properties seem to characterize essentially the superbrane actions. It should not come then as a suprise that $\kappa$-symmetric actions may also be introduced for $\mathrm{D} p$-branes, as in [8] for D-branes with rigid IIA and IIB superPoincaré symmetry. As is the case for ordinary $p$-branes, $\kappa$-symmetry is achieved when the relative coefficient of the kinetic and WZ-like part is such that the Bogomol'nyi bound is saturated.

Our extensions provide at the same time a connection between the CE cocycles and the mechanism of partial breaking of supersymmetry. The CE $(p+2)$-cocycles lead to (extended) loop-type or worldvolume current algebras (see, e.g. [12, 15, 54, 55, 56]) and the two-cocycle to the corresponding algebra of charges defining the extended superspace algebra. The new variables in the extended superspaces are also essential to define (invariant) actions. They may also be relevant in the problem of quantisation, the formulation of dualities (see Sec. 2.3.1) and in the formulation of the additional gauge freedom hidden in the definition of some superbrane fields, the worldvolume definition of which reflects an election of gauge. We suspect that the mathematical existence of the extensions considered here has a deeper meaning beyond the aspects discussed in this paper.

Note added. After completion of this paper an article [57] has appeared in hep-th, where an approach similar to that in Sec. 6.2 for the field $A$ is given for the IIB D-brane case.

Acknowledgements: This research has been partially supported by the research grant PB96-0756 from the DGICYT, Spain. C.C. wishes to thank the Spanish Ministry of Education and Culture for a post-doctoral fellowship and J.M.I acknowledges a grant from the Junta de Castilla y León. J.C.P.B. thanks the CSIC and the Ministerio de Educación for an FPI grant. Finally, the authors wish to thank Paul Townsend for helpful discussions.

\section{Appendix}

\section{A Non-central extensions}

We give here some details of the derivation of (3.5). After introducing $\Pi_{\mu_{1} \ldots \mu_{p-1} \alpha_{1}}$ satisfying (3.4), we look for non-trivial CE 2-cocycles with external indices $\left(\mu_{1} \ldots \mu_{p-2} \alpha_{1} \alpha_{2}\right)$. There are four available LI two-forms with these indices,

$$
\begin{aligned}
\rho^{(1)} & =\left(C \Gamma_{\nu \rho \mu_{1} \ldots \mu_{p-2}}\right)_{\alpha_{1} \alpha_{2}} \Pi^{\nu} \Pi^{\rho} \\
\rho^{(2)} & =\left(C \Gamma^{\nu}\right)_{\alpha_{1} \alpha_{2}} \Pi_{\nu \rho \mu_{1} \ldots \mu_{p-2}} \Pi^{\rho} \\
\rho^{(3)} & =\left(C \Gamma^{\nu}\right)_{\alpha_{1} \alpha_{2}} \Pi_{\nu \mu_{1} \ldots \mu_{p-2} \beta} \Pi^{\beta} \\
\rho^{(4)} & =\left(C \Gamma^{\nu}\right)_{\alpha_{1}^{\prime} \beta} \Pi_{\nu \mu_{1} \ldots \mu_{p-2} \alpha_{2}^{\prime}} \Pi^{\beta},
\end{aligned}
$$

none of which is closed. Looking for a linear combination $\rho \equiv \rho^{(1)}+\lambda_{2} \rho^{(2)}+\lambda_{3} \rho^{(3)}+\lambda_{4} \rho^{(4)}$ that is closed, we compute (making use of the MC equations for the available $\Pi$ 's)

$$
\begin{aligned}
d \rho= & \left\{2 a_{s}\left(C \Gamma_{\mu \nu \mu_{1} \ldots \mu_{p-2}}\right)_{\alpha_{1} \alpha_{2}}\left(C \Gamma^{\mu}\right)_{\gamma \delta}+\lambda_{2} a_{0}\left(C \Gamma^{\mu}\right)_{\alpha_{1} \alpha_{2}}\left(C \Gamma_{\mu \nu \mu_{1} \ldots \mu_{p-2}}\right)_{\gamma \delta}\right. \\
& \left.+\lambda_{3} a_{1}\left(C \Gamma^{\nu}\right)_{\alpha_{1} \alpha_{2}}\left(C \Gamma_{\mu \nu \mu_{1} \ldots \mu_{p-2}}\right)_{\gamma \delta}-\lambda_{4} a_{1}\left(C \Gamma^{\mu}\right)_{\alpha_{1}^{\prime} \gamma}\left(C \Gamma_{\mu \nu \mu_{1} \ldots \mu_{p-2}}\right)_{\alpha_{2}^{\prime} \delta}\right\} \Pi^{\nu} \Pi^{\gamma} \Pi^{\delta} \\
& +\left\{-\lambda_{2} \alpha_{1}\left(C \Gamma^{\mu}\right)_{\alpha_{1} \alpha_{2}}\left(C \Gamma^{\nu}\right)_{\gamma \delta}+\lambda_{3} \frac{a_{s} a_{1}}{a_{0}}\left(C \Gamma^{\nu}\right)_{\alpha_{1} \alpha_{2}}\left(C \Gamma^{\mu}\right)_{\gamma \delta}\right.
\end{aligned}
$$




$$
\left.+\lambda_{4} \frac{a_{s} a_{1}}{a_{0}}\left(C \Gamma^{\nu}\right)_{\alpha_{1}^{\prime} \gamma}\left(C \Gamma^{\mu}\right)_{\alpha_{2}^{\prime} \delta}\right\} \Pi_{\mu \nu \mu_{1} \ldots \mu_{p-2}} \Pi_{\gamma} \Pi_{\delta}
$$

Inside the first curly brackets above, one can combine the third term with the second, changing at the same time its sign (the $\Gamma$ 's are antisymmetric in the vector indices). In the fourth term, one can also symmetrise over $\gamma, \delta$ (since $\Pi_{\gamma}$ and $\Pi_{\delta}$ commute). Effecting explicitly this symmetrisation, as well as the indicated one (by the primes) over $\alpha_{1}, \alpha_{2}$, one gets four terms, which, together with the other two, give exactly the six permutations of (3.3) (the $\Gamma$ 's are symmetric in the spinorial indices, so that the twenty-four permutations of (3.3) reduce to six). The sum of all six terms will be zero (due to (3.3)) if their coefficients are equal - this gives the equations

$$
a_{0} \lambda_{2}-a_{1} \lambda_{3}=2 a_{s} \quad, \quad-\frac{1}{4} a_{1} \lambda_{4}=2 a_{s} \quad .
$$

Inside the second curly brackets in (A.2), the last term is zero because of antisymmetry in $\mu, \nu$. The sum of the first two will be zero (for the same reason) if their coefficients are equal, i.e. if

$$
a_{s} \lambda_{2}+\frac{a_{s} a_{1}}{a_{0}} \lambda_{3}=0
$$

Solving the linear system of (A.3), (A.4) one gets

$$
\lambda_{2}=\frac{a_{s}}{a_{0}} \quad, \quad \lambda_{3}=-\frac{a_{s}}{a_{1}} \quad, \quad \lambda_{4}=-8 \frac{a_{s}}{a_{1}},
$$

which leads to the first of (3.5).

For the next extension, looking for LI two-forms with indices $\left(\mu_{1} \ldots \mu_{p-3} \alpha_{1} \alpha_{2} \alpha_{3}\right)$ we find

$$
\begin{aligned}
\rho^{(1)} & =\left(C \Gamma^{\nu}\right)_{\alpha_{1}^{\prime} \alpha_{2}^{\prime}} \Pi_{\nu \rho \mu_{1} \ldots \mu_{p-3} \alpha_{3}^{\prime}} \Pi^{\rho} \\
\rho^{(2)} & =\left(C \Gamma^{\nu}\right)_{\alpha_{1}^{\prime} \beta} \Pi_{\nu \mu_{1} \ldots \mu_{p-3} \alpha_{2}^{\prime} \alpha_{3}^{\prime}} \Pi^{\beta} \\
\rho^{(3)} & =\left(C \Gamma^{\nu}\right)_{\alpha_{1}^{\prime} \alpha_{2}^{\prime}} \Pi_{\nu \mu_{1} \ldots \mu_{p-3} \beta \alpha_{3}^{\prime}} \Pi^{\beta},
\end{aligned}
$$

none of which is closed (we use, for simplicity, the same symbols $\rho, \lambda$ as in the previous extension). For their linear combination $\rho \equiv \rho^{(1)}+\lambda_{2} \rho^{(2)}+\lambda_{3} \rho^{(3)}$ we compute

$$
\begin{aligned}
d \rho= & \left.\left(C \Gamma^{\nu}\right)_{\alpha_{1}^{\prime} \alpha_{2}^{\prime}} a_{1}\left\{\left(C \Gamma_{\lambda \nu \rho \mu_{1} \ldots \mu_{p-3}}\right)_{\beta \alpha_{3}^{\prime}} \Pi^{\lambda} \Pi^{\beta}+\frac{a_{s}}{a_{0}}\left(C \Gamma^{\sigma}\right)_{\beta \alpha_{3}^{\prime}} \Pi_{\sigma \nu \rho \mu_{1} \ldots \mu_{p-3}}\right) \Pi^{\beta}\right\} \Pi^{\rho} \\
& -a_{s}\left(C \Gamma^{\nu}\right)_{\alpha_{1}^{\prime} \alpha_{2}^{\prime}}\left(C \Gamma^{\nu}\right)_{\gamma \delta} \Pi_{\nu \rho \mu_{1} \ldots \mu_{p-3} \alpha_{3}^{\prime}} \Pi^{\gamma} \Pi^{\delta} \\
& +\lambda_{2} a_{4}\left(C \Gamma^{\nu}\right)_{\alpha_{1}^{\prime} \beta}\left\{\left(C \Gamma_{\sigma \rho \nu \mu_{1} \ldots \mu_{p-3}}\right)_{\alpha_{2}^{\prime} \alpha_{3}^{\prime}} \Pi^{\sigma} \Pi^{\rho}+\frac{a_{s}}{a_{0}}\left(C \Gamma^{\sigma}\right)_{\alpha_{2}^{\prime} \alpha_{3}^{\prime}} \Pi_{\sigma \rho \nu \mu_{1} \ldots \mu_{p-3}} \Pi^{\rho}\right. \\
& \left.-\frac{a_{s}}{a_{1}}\left(C \Gamma^{\sigma}\right)_{\alpha_{2}^{\prime} \alpha_{3}^{\prime}} \Pi_{\sigma \nu \mu_{1} \ldots \mu_{p-3} \gamma} \Pi^{\gamma}-8 \frac{a_{s}}{a_{1}}\left(C \Gamma^{\sigma}\right)_{\alpha_{2}^{\prime} \gamma} \Pi_{\sigma \nu \mu_{1} \ldots \mu_{p-3} \alpha_{3}^{\prime}} \Pi^{\gamma}\right\} \Pi^{\beta} \\
& +\lambda_{3} a_{2}\left(C \Gamma^{\nu}\right)_{\alpha_{1}^{\prime} \alpha_{2}^{\prime}}\left\{\left(C \Gamma_{\sigma \rho \nu \mu_{1} \ldots \mu_{p-3}}\right)_{\alpha_{2}^{\prime} \alpha_{3}^{\prime}} \Pi^{\sigma} \Pi^{\rho}+\frac{a_{s}}{a_{0}}\left(C \Gamma^{\sigma}\right)_{\beta \alpha_{3}^{\prime}} \Pi_{\sigma \rho \nu \mu_{1} \ldots \mu_{p-3}} \Pi^{\rho}\right. \\
& \left.-\frac{a_{s}}{a_{1}}\left(C \Gamma^{\sigma}\right)_{\beta \alpha_{3}^{\prime}} \Pi_{\sigma \nu \mu_{1} \ldots \mu_{p-3} \gamma} \Pi^{\gamma}-8 \frac{a_{s}}{a_{1}}\left(C \Gamma^{\sigma}\right)_{\bar{\beta} \gamma} \Pi_{\sigma \nu \mu_{1} \ldots \mu_{p-3} \bar{\alpha}_{3}} \Pi^{\gamma}\right\} \Pi^{\beta}
\end{aligned}
$$

(the barred indices in the last term denote a second symmetrisation, besides the one over the primed indices). There is a novelty here compared with the previous extension: there are four different types of terms in the $\Pi$ 's, the coefficients of which must separately vanish, giving rise to four linear equations for the two unknowns $\lambda_{2}, \lambda_{3}$ (care must be taken of the fact that when the second symmetrisation in the last term above is effected, corresponding to the barred indices, one obtains contributions to two different types of terms in the П's). Making use of (3.3) and of the symmetry properties of the $\Gamma$ 's, as in the previous extension, one arrives at the (overdetermined) linear system

$$
\lambda_{2}-\lambda_{3}=\frac{a_{1}}{a_{2}} \quad, \quad 4 \frac{a_{s} a_{2}}{a_{1}} \lambda_{3}=a_{s} \quad, \quad \lambda_{2}-5 \lambda_{3}=0
$$

(the first equation appears twice) which nevertheless admits the solution

$$
\lambda_{2}=\frac{5 a_{1}}{4 a_{2}} \quad, \quad \lambda_{3}=\frac{a_{1}}{4 a_{2}},
$$

leading to the second of (3.5). The last of $(3.5)$, as well as $(3.6)$, are proved similarly. 


\section{B $\quad D=10 \Gamma$-matrix identities}

We prove here the $\Gamma$-identities needed in Sec. 6.1 ${ }^{16}$. The first two identities in (6.9) follow by dimensional reduction from the known $D=11$ relations

$$
\begin{aligned}
\left(C \Gamma^{\tilde{\mu}_{2}}\right)_{\alpha^{\prime} \beta^{\prime}}\left(C \Gamma_{\tilde{\mu}_{1} \tilde{\mu}_{2}}\right)_{\delta^{\prime} \epsilon^{\prime}} & =0 \\
\left(C \Gamma^{\tilde{\mu}_{5}}\right)_{\alpha^{\prime} \beta^{\prime}}\left(C \Gamma_{\tilde{\mu}_{1} \ldots \tilde{\mu}_{5}}\right)_{\delta^{\prime} \epsilon^{\prime}}-3\left(C \Gamma_{\left[\tilde{\mu}_{1} \tilde{\mu}_{2}\right.}\right)_{\alpha^{\prime} \beta^{\prime}}\left(C \Gamma_{\left.\tilde{\mu}_{3} \tilde{\mu}_{4}\right]}\right)_{\delta^{\prime} \epsilon^{\prime}} & =0
\end{aligned} .
$$

where the tilded indices $\tilde{\mu}=0,1, \ldots, 10$.

The third identity can be proved as follows. First, using that

$$
\Gamma_{\mu_{1} \ldots \mu_{6}}=\Gamma_{\mu_{1} \ldots \mu_{5}} \Gamma_{\mu_{6}}-5 \Gamma_{\left[\mu_{1} \ldots \mu_{4}\right.} \eta_{\left.\mu_{5}\right] \mu_{6}}
$$

and the fact that $\Gamma_{11}^{2}=1$, we see that

$$
\left(C \Gamma^{\mu_{6}}\right)_{\alpha^{\prime} \beta^{\prime}}\left(C \Gamma_{\mu_{1} \ldots \mu_{6}}\right)_{\delta^{\prime} \epsilon^{\prime}}=\left(C \Gamma^{\mu_{6}}\right)_{\alpha^{\prime} \beta^{\prime}}\left(C \Gamma_{\mu_{1} \ldots \mu_{5}} \Gamma_{11}\right)_{\delta^{\prime} \lambda}\left(\Gamma_{11} \Gamma_{\mu_{6}}\right)_{\epsilon^{\prime}}^{\lambda}
$$

since the second term in $(\overline{\mathrm{B} .2})$ does not contribute because $\left(C \Gamma_{\mu_{1} \ldots \mu_{4}}\right)_{\delta \epsilon}$ is antisymmetric (primed indices are symmetrised). Now, due to the identity $\left(C \Gamma_{\mu}\right)_{\alpha^{\prime} \beta^{\prime}}\left(C \Gamma_{11} \Gamma^{\mu}\right)_{\delta^{\prime} \epsilon^{\prime}}=0$, we have $\left(C \Gamma_{\mu}\right)_{\alpha^{\prime} \beta^{\prime}}\left(\Gamma_{11} \Gamma^{\mu}\right)_{\epsilon^{\prime}}^{\lambda}=-\left(\Gamma_{\mu}\right)_{\alpha^{\prime}}^{\lambda}\left(\Gamma_{11} \Gamma^{\mu}\right)_{\beta^{\prime} \epsilon^{\prime}}$ so that

$$
\begin{aligned}
\left(C \Gamma^{\mu_{6}}\right)_{\alpha^{\prime} \beta^{\prime}}\left(C \Gamma_{\mu_{1} \ldots \mu_{6}}\right)_{\delta^{\prime} \epsilon^{\prime}} & =-\left(C \Gamma_{\mu_{1} \ldots \mu_{5}} \Gamma_{11} \Gamma^{\mu_{6}}\right)_{\delta^{\prime} \alpha^{\prime}}\left(\Gamma_{11} \Gamma_{\mu_{6}}\right)_{\beta^{\prime} \epsilon^{\prime}} \\
& =5\left(C \Gamma_{\left[\mu_{1} \ldots \mu_{4}\right.} \Gamma_{11}\right)_{\delta^{\prime} \alpha^{\prime}}\left(C \Gamma_{11} \Gamma_{\left.\mu_{5}\right]}\right)_{\beta^{\prime} \epsilon^{\prime}},
\end{aligned}
$$

where in the second equality use has been made of $(\overline{B .2})$ and the fact that $\left(C \Gamma_{\mu_{1} \ldots \mu_{6}} \Gamma_{11}\right)_{\delta \alpha}$ is antisymmetric.

Finally, the fourth equation in (6.9) may be shown to be equivalent to the second. Indeed, by multiplying the fourth identity by $\epsilon^{\mu_{1} \ldots \mu_{7} \nu_{1} \nu_{2} \nu_{3}}$ and using that $\Gamma_{\mu_{1} \ldots \mu_{q}} \Gamma_{11} \propto \frac{1}{(10-q) !} \epsilon_{\mu_{1} \ldots \mu_{q} \rho_{1} \ldots \rho_{10-q}} \Gamma^{\rho_{1} \ldots \rho_{10-q}}$, one obtains

$$
\frac{7 ! 3 !}{2}\left(C \Gamma^{\left[\nu_{1}\right.}\right)_{\alpha^{\prime} \beta^{\prime}}\left(C \Gamma^{\left.\nu_{2} \nu_{3}\right]}\right)_{\delta^{\prime} \epsilon^{\prime}}+\frac{6 ! 4 ! 7}{4 !}\left(C \Gamma^{\mu_{7} \nu_{1} \nu_{2} \nu_{3}} \Gamma_{11}\right)_{\alpha^{\prime} \beta^{\prime}}\left(C \Gamma_{\mu 7} \Gamma_{11}\right)_{\delta^{\prime} \epsilon^{\prime}}=0
$$

which is equivalent to the second equation in (B.1) due to the fact that $i \Gamma_{\mu} \Gamma_{11}$ realize the same Clifford algebra as $\Gamma_{\mu}$.

\section{References}

[1] A. Achúcarro, J. M. Evans, P. K. Townsend and D. L. Wiltshire, Super p-branes, Phys. Lett. 198B, 441-446 (1987).

[2] J. A. de Azcárraga and P. K. Townsend, Superspace geometry and classification of supersymmetric extended objects, Phys. Rev. Lett. 62, 2579-2512 (1989).

[3] C. Chevalley and S. Eilenberg, Cohomology theory of Lie groups and Lie algebras, Trans. Am. Math. Soc. 63, 85-124 (1948).

[4] M. J. Duff, R. R. Khuri and J. X. Lu, String solitons, Phys. Rep. 259, 213-326 (1995).

[5] M. J. Duff, Supermembranes, CTP-TAMU-61/96, hep-th/9611203.

[6] J. Polchinski, Dirichlet branes and Ramond-Ramond charges, Phys. Rev. Lett. 75, 4724-4727 (1995)

[7] J. Polchinski, S. Chaudhuri and C. V. Johnson, Notes on D-branes, hep-th/9602052.

[8] M. Aganagic, C. Popescu and J. H. Schwarz, D-brane actions with local א-symmetry, Phys. Lett. B393, 311-315 (1997).

\footnotetext{
${ }^{16}$ In the search for further possible $\Gamma$-identities the results in 58 may be useful.
} 
[9] P. S. Howe, O. Raetzel, I. Rudychev and E. Sezgin, L-branes, KCL-MTH-98-39 / CPT-TAMU3498, hep-th/9810081.

[10] J. H. Schwarz, Lectures on superstring and $M$-theory dualities, Nucl. Phys. Proc. Suppl. 55B, 1 (1997).

[11] T. Banks, W. Fischler, S. H. Shenker and L. Susskind, M theory as a matrix model: a conjecture, Phys. Rev. D55, 5112-5128 (1997).

[12] J. A. de Azcárraga, J. P. Gauntlett, J. M. Izquierdo and P. K. Townsend, Topological extensions of the supersymmetry algebra for extended objects, Phys. Rev. Lett. 63, 2443-2446 (1989).

[13] M. B. Green, Super-translations, superstrings and Chern-Simons forms, Phys. Lett. B223, 157$164(1989)$.

[14] E. Bergshoeff and E. Sezgin, New spacetime superalgebras and their Kac-Moody extensions, Phys. Lett. B232, 96-103 (1989).

[15] J. A. de Azcárraga, J. M. Izquierdo and P. K. Townsend, Classical anomalies of supersymmetric extended objects, Phys. Lett. B267, 366-373 (1991).

[16] E. Bergshoeff and E. Sezgin, Super p-brane theories and new spacetime superalgebras, Phys. Lett. B354, 256-263 (1995).

[17] I. Bars, Supersymmetry, p-brane duality and hidden spacetime dimensions, Phys. Rev. D54, 5202-5210 (1996).

[18] D. Sorokin and P. K. Townsend, $M$-theory superalgebra from the $M$-five-brane, Phys. Lett. B412, 265-273 (1997).

[19] E. Sezgin, The M-algebra, Phys. Lett. B392, 323-331 (1997).

[20] A. Deriglazov and A. Galajinsky, A linear realization for the new spacetime superalgebras in 10 and 11 dimensions, Mod. Phys. Lett. A12, 1517-1529 (1997).

[21] I. Bars, S-theory, Phys. Rev. D55, 2373-2381 (1997).

[22] M. Sakaguchi, Type II superstrings and new spacetime superalgebras, hep-th/9809113.

[23] I. Bandos, P. Pasti, D. Sorokin, M. Tonin and D. Volkov, Superstrings and supermembranes in the doubly supersymmetrical approach, Nucl. Phys. B446, 79-119 (1995)

[24] P.S. Howe and E. Sezgin, Superbranes, Phys. Lett. B390, 133-142 (1997)

[25] V. Aldaya and J. A. de Azcárraga, A note on the meaning of covariant derivatives in supersymmetry, J. Math. Phys. 26, 1818-1821 (1985).

[26] J. A. de Azcárraga and J. M. Izquierdo, Lie groups, Lie algebras, cohomology and some applications in physics (Camb. Univ. Press, 1995).

[27] R. Haag, J. T. Lopuszański and M. Sohnius, All possible generators of supersymmetry of the S-matrix, Nucl. Phys. B88, 257-274 (1975).

[28] J. W. van Holten and A. Van Proeyen, $N=1$ supersymmetry algebras in d=2, 3, 4 mod 8, J. Phys. A15, 3763-3783 (1982).

[29] R. D'Auria and P. Fré, Geometric supergravity in D=11 and its hidden supergroup, Nucl. Phys. B201, 101-140 (1982) (E.: ibid. B206, 496 (1982))

[30] P. A. Zizzi, An extesion of the Kaluza-Klein picture for the $N=4$ supersymmetric Yang-Mills theory, Phys. Lett. 137B, 57-61 (1984). 
[31] P. A. Zizzi, Antisymmetric tensors in supersymmetric algebras and spontaneous compactification, Phys. Lett. 149B, 333-336 (1984).

[32] J. A. de Azcárraga, Wess-Zumino terms, extended algebras and anomalies in classical physics, Contemp. Math. 132, 75-98 (1992).

[33] W. Siegel, Randomizing the superstring, Phys. Rev. D50, 2799-2805 (1994).

[34] F. Berezin, The mathematical basis of supersymmetric theories, Sov. J. Nucl. Phys. 29, 857-866 (1979) [Yad. Fiz. 29, 1670] (Section 5).

[35] A. S. Schwarz, Supergravity and field space democracy, Nucl. Phys. B 171, 154-156 (1980)

[36] A. V. Gayduk, V. N. Romanov and A. S. Schwarz, Supergravity and field space democracy, Commun. Math. Phys. 79, 507-528 (1981).

[37] V. Aldaya and J. A. de Azcárraga, Geometric quantisation in the presence of an electromagnetic field, Int. J. Theor. Phys. 22, 1-18 (1983).

[38] H. Hammer, Topological extensions of Noether charge algebras carried by Dp-branes, Nucl. Phys. B521, 503-546 (1998).

[39] D. Sullivan, Infinitesimal computations in topology, Ins. des Haut. Étud. Sci., Pub. Math. 47, 269-331 (1977).

[40] R. D'Auria, P. Fré and T. Regge Graded Lie algebra, cohomology and supergravity, Riv. Nuov. Cim. 3, fasc. 12 (1980).

[41] P. van Nieuwenhuizen, Free graded differential superalgebras, in Lect. Notes in Phys. 180, 228-245, Springer-Verlag (1983).

[42] P. Van Nieuwenhuizen, An introduction to simple gravity and the Kaluza-Klein program, in Relativité, groupes et topologie II, B. S. De Witt and R. Stora (eds.), pp. 823-932 (Elsevier, 1984).

[43] P. C. West, Supergravity, brane dynamics and string duality, 1997 Isaac Newton lectures, KCLMTH-98-55, hep-th/9811101.

[44] P. K. Townsend, p-brane democracy, in Particles, strings and cosmology, J. Bagger, G. Domokos, A. Falk and A. Kovesi-Domokos (eds.), pp. 271-285 (World Sci., 1996), hep-th/9507048.

[45] P. K. Townsend, $M$ theory from its superalgebra, 1997 Cargèse lectures, hep-th/9712004.

[46] A. Giveon, M. Porrati and E. Rabinovici, Target space duality in string theory, Phys. Rep. 244, 77-202 (1994).

[47] J. M. Evans, Super p-brane Wess-Zumino terms, Class. Quant. Grav. 5, L87-L90 (1988).

[48] E. Bergshoeff and P.K. Townsend, Super D-branes revisited, Nucl. Phys. B531, 226-238 (1998).

[49] P. K. Townsend, Worldsheet electromagnetism and the superstring tension, Phys. Lett. B272, 285-288 (1992).

[50] J. A. de Azcárraga, J. M. Izquierdo and P. K. Townsend, Kaluza-Klein origin for the superstring tension, Phys. Rev. D45, R3321-R3325 (1992).

[51] E. Bergshoeff, L.A.J. London and P.K. Townsend, Spacetime invariance and the super p-brane, Class. Quantum Grav. 9, 2545-2556 (1992).

[52] I. Bandos, K. Lechner, A.Nurmagambetov, P. Pasti, D.Sorokin and M. Tonin, Covariant action for the super-five-brane of M-theory, Phys. Rev. Lett. 78, 4332-4434 (1997). 
[53] M. Aganagic, J. Park, C. Popescu and J. H. Schwarz, Worldvolume action of the M-theory fivebrane, Nucl. Phys. B496, 191-214 (1997)

[54] E. Bergshoeff, F. Delduc and E. Sokatchev, Light-like integrability in loop superspace, Kac-Moody central charges and Chern-Simons terms, Phys. Lett. B262, 444-450, (1991)

[55] E. Bergshoeff, P.S. Howe, C.N. Pope, E. Sezgin and E. Sokatchev, Ten-dimensional supergravity from light-like integrability in loop superspace, Nucl. Phys. B354, 113-128 (1991)

[56] E. Bergshoeff, R. Percacci, E. Sezgin, K.S. Stelle and P.K. Townsend, U(1)-Extended gauge algebras in p-loop space, Nucl. Phys. B398, 343-358 (1993)

[57] M. Abe, M. Hatsuda, K. Kamimura and T. Tokunaga, SO $(2,1)$ covariant IIB superalgebra, hepth/9903234.

[58] S. Naito, K. Osada and T. Fukui, Fierz identities and invariance of 11-dimensional supergravity action, Phys. Rev. D34, 536-552 (1986) 